\title{
Access to Polycyclic Indole-3,4-Fused Nine-Membered Ring via Cascade 1,6-Hydride Transfer/Cyclization
}

\author{
Shuo Yang, ${ }^{\dagger}$ Xiao-De An, ${ }^{*}$, Bin Qiu $^{\dagger}{ }^{\text {Rui-Bin Liu }},{ }^{\perp} \mathrm{Jian}$ Xiao $*, \dagger, *$ \\ ${ }^{\dagger}$ College of Chemistry and Pharmaceutical Sciences, Qingdao Agricultural University, Qingdao \\ 266109, China. \\ $\$$ College of Marine Science and Engineering, Qingdao Agricultural University, Qingdao, 266109, \\ China. \\ ${ }^{\perp}$ Shandong Kangqiao Biotechnology Co. Ltd., Binzhou 256500, China. \\ E-mail: axdchem@163.com; chemjianxiao@163.com.
}

\section{Supporting Information}

\section{Table of Contents}

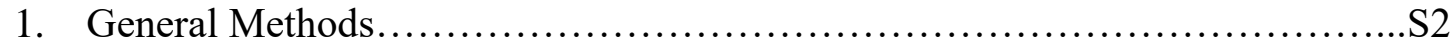

2. List of the Starting Materials ............................................. 3

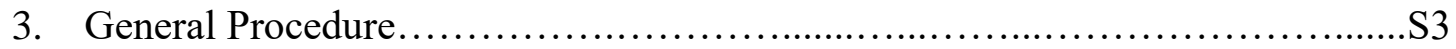

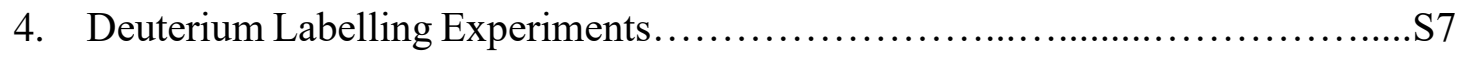

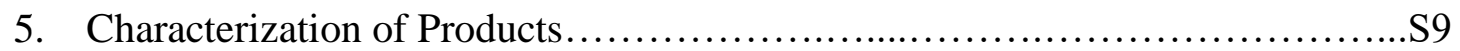

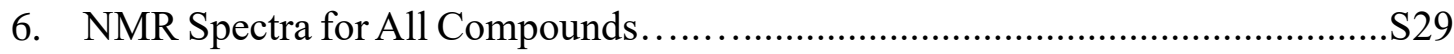

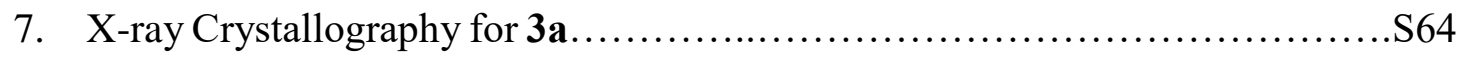




\section{General Methods.}

All commercially available reagents and solvents, unless otherwise indicated, were used without further purification. Reactions were monitored by thin layer chromatography (TLC) with $0.2 \mathrm{~mm}$ silica gel-coated HSGF 254 plates, visualized by UV light at 254 or $365 \mathrm{~nm}$. Products were isolated and purified by column chromatography on 200-300 mesh silica gel. For reactions that require heating, the heat source is heating mantle. ${ }^{1} \mathrm{H},{ }^{13} \mathrm{C}$ and ${ }^{19} \mathrm{~F}$ NMR spectra were recorded on a Bruker AMX $500\left(500 \mathrm{MHz}\right.$ for ${ }^{1} \mathrm{H}, 126 \mathrm{MHz}$ for ${ }^{13} \mathrm{C}$ and $471 \mathrm{MHz}$ for ${ }^{19} \mathrm{~F}$ NMR) spectrometer at room temperature. The chemical shifts $(\delta)$ were reported in ppm with respect to an internal standard, tetramethylsilane $(0 \mathrm{ppm})$, and the solvent $\left(\mathrm{CDCl}_{3},{ }^{1} \mathrm{H}: \delta=7.26 \mathrm{ppm}\right.$, $\left.{ }^{13} \mathrm{C}: \delta=77.16 \mathrm{ppm}\right)$. Coupling constants $(J)$ are given in Hertz. Splitting patterns of apparent multiplets associated with an averaged coupling constants were designated as s (singlet), d (doublet), t (triplet), q (quartet), m (multiplet), dd (doublet of doublets) and br (broadened). All ${ }^{13} \mathrm{C}$ spectra were recorded with broadband proton decoupling. HRMS were performed on a Waters XEVO QTOF mass spectrometer and Thermo Fisher Orbitrap Fusion Lumos spectrometer. The X-ray diffraction analysis were performed on Gemini E/EOS. The X-ray source used for the single crystal X-ray diffraction analysis of compound $\mathbf{3 a}$ was $\mathrm{CuKa}(1=1.54178)$, and the thermal ellipsoid was drawn at the $30 \%$ probability level. 


\section{List of the Starting Materials.}

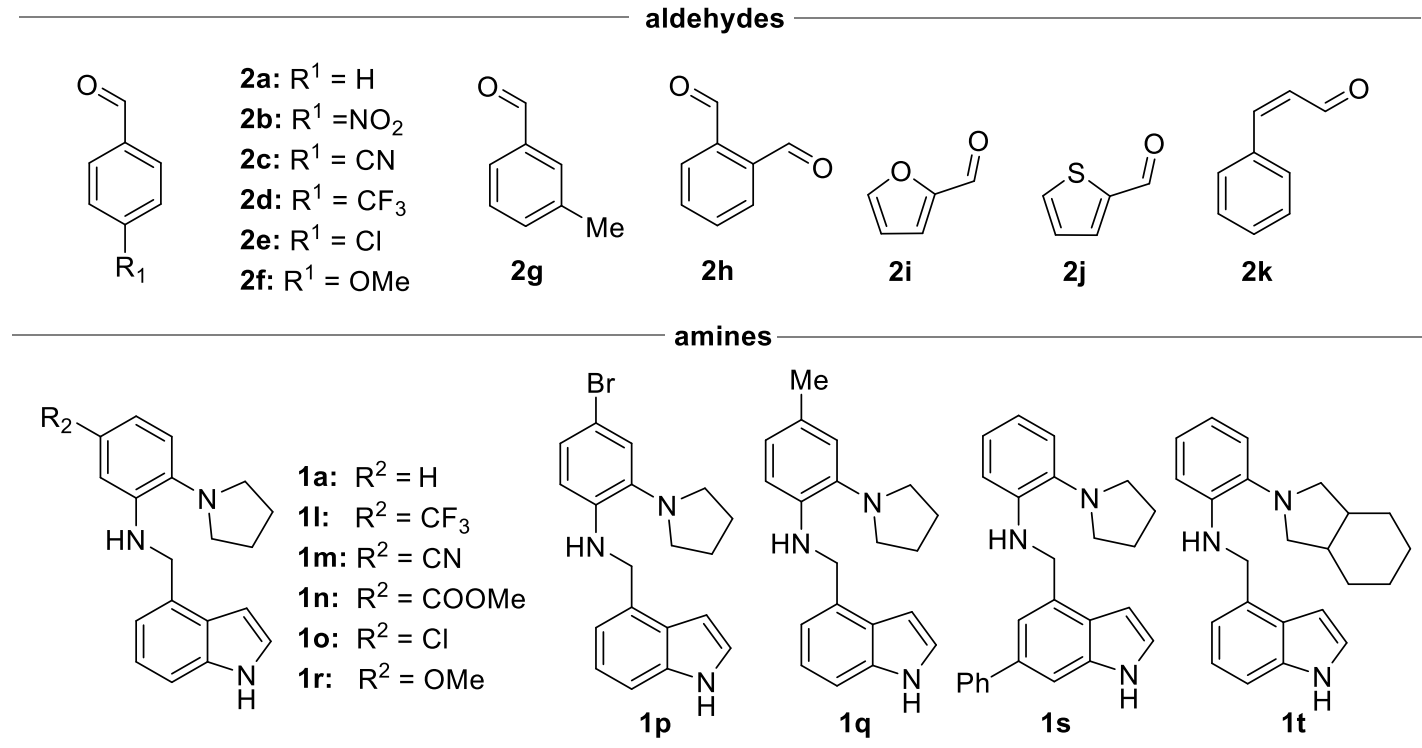

\section{General Procedure.}

3.1 General procedure for the the synthesis of $N-((1 \mathrm{H}-$ indol-4-yl $)$ methyl)-2-(pyrrolidin1-yl)anilines 1a-t. ${ }^{1-3}$

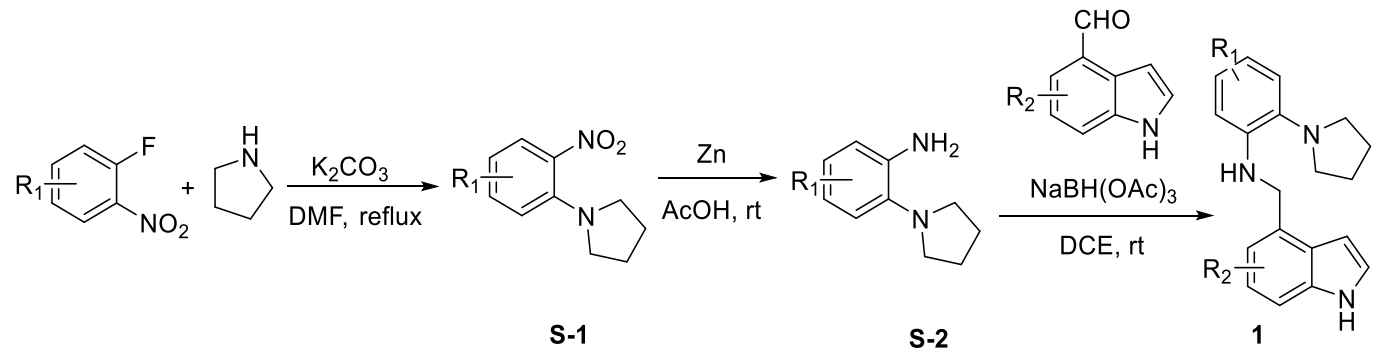

To a stirred solution of 1-fluoro-2-nitrobenzene $(5.0 \mathrm{mmol})$ and potassium carbonate (10.0 mmol, 2 equiv) in DMF (20 mL) was added the pyrrolidine ( $6.0 \mathrm{mmol}, 1.2$ equiv). The mixture was refluxed until completion of the reaction as indicated by TLC analysis. After cool to room temperature, the mixture was diluted with water $(5 \mathrm{~mL})$, and extracted with ethyl acetate $(2 \times 25 \mathrm{~mL})$. The combined organic layers were washed with a saturated brine $(25 \mathrm{~mL})$ and dried with $\mathrm{Na}_{2} \mathrm{SO}_{4}$. After concentrated under

\footnotetext{
1 Murarka, S.; Zhang, C.; Konieczynska, M. D.; Seidel, D. Org. Lett. 2009, 11, 129.

${ }^{2}$ Kroemer, J.; Kirkpatrick, C.; Maricle, B.; Gawrych, R.; Mosher, M. D.; Kaufman, D. Tetrahedron Lett. 2006, $47,6339$.

3 Wang, D. M.; Sun, M. N.; Liu, G. J. Comb. Chem. 2009, 11, 556.
} 
reduced pressure, the residue was purified by flash column chromatography on silica gel to afford the desired product $\mathbf{S - 1}$.

To a stirred solution of $\mathbf{S - 1}(4.3 \mathrm{mmol})$ in $\mathrm{AcOH}(8 \mathrm{~mL})$ was added zinc powder $(21.5$ mmol, 5 equiv) portion-wise for 10 minutes. The reaction mixture was then stirred at room temperature until completion of the reaction as indicated by TLC analysis. The solution was filtered and evaporated at reduced pressure. The residue was basified with aqueous $\mathrm{NaHCO}_{3}$ solution, extracted with ethyl acetate $(3 \times 25 \mathrm{~mL})$ and subsequently dried with $\mathrm{Na}_{2} \mathrm{SO}_{4}$. After evaporation of the solvent, the crude product was directly purified by flash column chromatography on silica gel to afford the desired product $\mathbf{S}$ 2.

To a stirred solution of S-2 $(2.5 \mathrm{mmol})$ in DCE $(10 \mathrm{~mL})$ was added the indol-aldehyde (2.8 mmol). Then, $\mathrm{NaBH}(\mathrm{OAc})_{3}(5.0 \mathrm{mmol})$ was added to the stirred solution, then the mixture was stirred at room temperature until completion of the reaction as indicated by TLC analysis. The solution was basified with aqueous $\mathrm{NaHCO}_{3}$ solution, extracted with ethyl acetate $(3 \times 25 \mathrm{~mL})$ and subsequently dried with $\mathrm{Na}_{2} \mathrm{SO}_{4}$. After evaporation of the solvent, the crude product was directly purified by flash column chromatography on silica gel to afford the desired product $\mathbf{1}$. 
3.2 General procedure for the synthesis of deuterated $N-((1 \mathrm{H}-\mathrm{indol}-4-\mathrm{yl})$ methyl)-2(pyrrolidin-1-yl-2,2,5,5-d4)aniline $[\mathbf{D}]-\mathbf{1} \mathbf{a}^{4,5}$ and $[\mathbf{D}]-\mathbf{1} \mathbf{b} .^{6,7}$

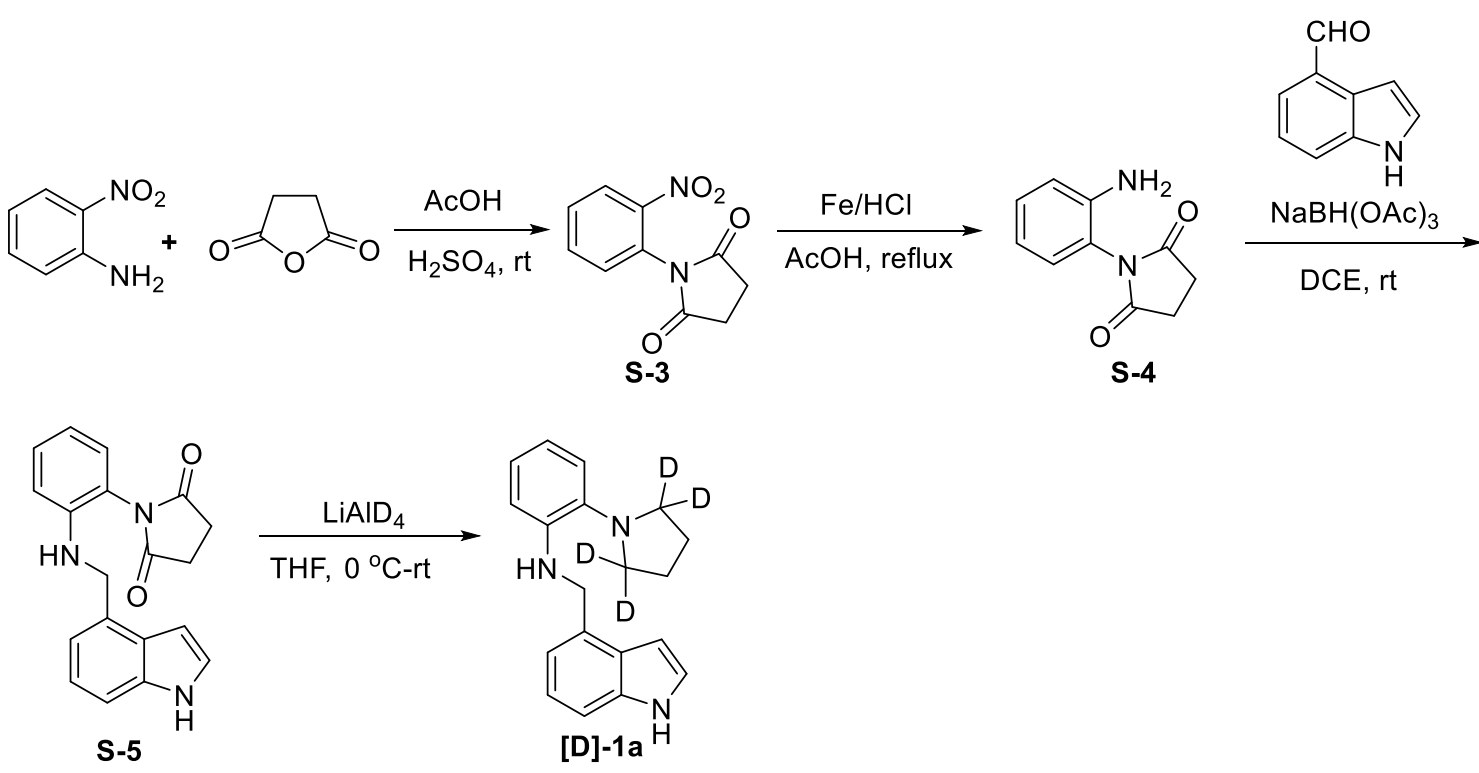

To a stirred solution of the 2-nitroaniline $(5.5 \mathrm{~g}, 40.0 \mathrm{mmol})$ in acetic acid $(30 \mathrm{~mL})$, the anhydride ( $4.4 \mathrm{~g}, 44.0 \mathrm{mmol})$ was added in one portion. Then, the reaction mixture was stirred for $10 \mathrm{~min}$ at $45^{\circ} \mathrm{C}$. After the clear solution turned into slurry, the sulfuric acid $(9.2 \mathrm{~g}, 93.8 \mathrm{mmol})$ was added in one portion. the suspension was stirred at $60{ }^{\circ} \mathrm{C}$ for $30 \mathrm{~min}$. Then, the cooled mixture was poured into ice. The precipitates formed were filtered, washed with aqueous $\mathrm{Na}_{2} \mathrm{CO}_{3}$ solution and water. The resulting solid recrystallized from aqueous ethanol to afford the desired product S-3.

To a solution of $\mathbf{S - 3}(10 \mathrm{mmol})$ in $80 \mathrm{~mL}$ acetone was added acetic acid $(8 \mathrm{~mL})$, water $(8 \mathrm{~mL})$ and iron powder $(6.7 \mathrm{~g}, 120 \mathrm{mmol})$. The reaction mixture was refluxed for $8 \mathrm{~h}$. The reaction was cooled and filtered over pad of celite. The filtrate was concentrated under vacuum. The crude product was directly purified by flash column chromatography on silica gel (eluent: ethyl acetate/petroleum ether $=1: 2$ ) to afford the desired product S-4.

\footnotetext{
${ }^{4}$ Patil, S. V.; Mahale, K. A.; Gosavi, K. S.; Deshmukh, G. B.; Patil, N. S. Org. Prep. Proced. Int. 2013, 45, 314.

${ }^{5}$ K. Maji ; M. Abbasi ; D. Podder ; R. Datta ; D. Haldar. ChemistrySelect. 2018, 3, 10220.

${ }^{6}$ Tanaka, Y.; Kurasawa, O.; Yokota, A.; Klein, M. G.; Ono, K.; Saito, B.; Matsumoto, S.; Okaniwa, M.; Ambrus-Aikelin, G.; Morishita,

D.; Kitazawa, S.; Uchiyama, N.; Ogawa, K.; Kimura, H.; Imamura, S. J. Med. Chem. 2020, 63, 3215.

${ }^{7}$ Hendrick, C. E.; McDonald, S. L.; Wang, Q. Org. Lett. 2013, 15, 3444.
} 
The synthesis method to access $\mathbf{S - 5}$ was according to the preparation of compound 1.

To S-5 (760 mg, $2.4 \mathrm{mmol})$ in $10 \mathrm{~mL}$ THF at $0{ }^{\circ} \mathrm{C}$ was added $\mathrm{LiAlD}_{4}(504 \mathrm{mg}, 12.0$ mmol, 5 equiv). The reaction mixture was then refluxed until completion of the reaction as indicated by TLC analysis. The reaction was quenched by the dropwise addition of $\mathrm{H}_{2} \mathrm{O}$. After evaporation of the solvent, the residue was directly purified by flash column chromatography on silica gel (eluent: ethyl acetate/dichloromethane $=1: 5$ ) to afford the desired product [D]-1a.

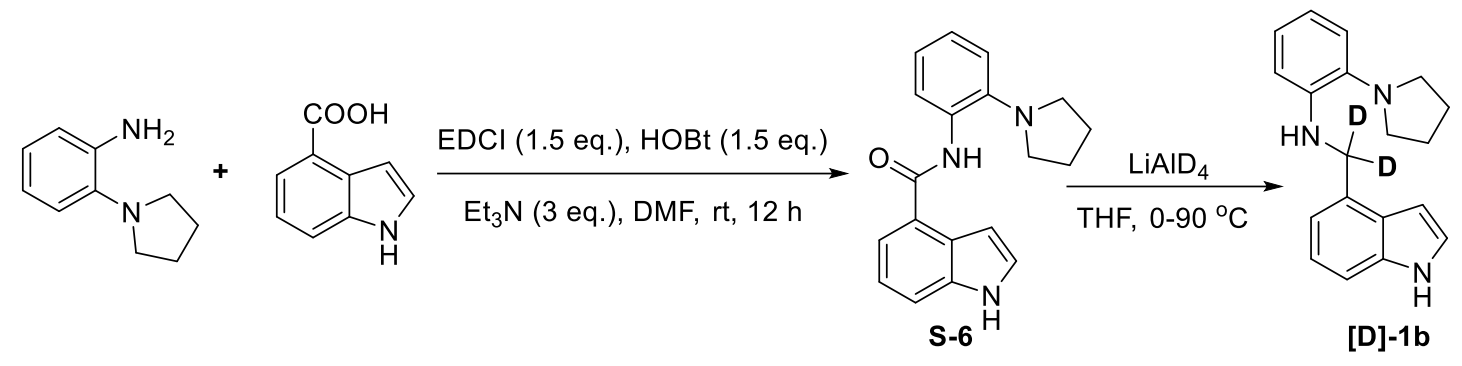

To a solution of indole-4-carboxylic acid (1.6 g, $10 \mathrm{mmol})$ and diamine (1.9 g, 12 $\mathrm{mmol})$ in DMF (13 mL), EDC-HCl (2.9 g, $15 \mathrm{mmol}), \mathrm{HOBt}-\mathrm{H}_{2} \mathrm{O}(2.0 \mathrm{~g}, 15 \mathrm{mmol})$, and $\mathrm{Et}_{3} \mathrm{~N}(3.1 \mathrm{~g}, 30 \mathrm{mmol})$ were added at $0{ }^{\circ} \mathrm{C}$. The mixture was stirred at room temperature overnight. The reaction mixture was diluted with water and extracted with EtOAc. The organic layer was washed with brine, dried over $\mathrm{Na}_{2} \mathrm{SO}_{4}$, and concentrated in vacuo. The residue was purified by column chromatography (eluent: ethyl acetate/petroleum ether $=1: 10)$ to give $\mathbf{S - 6}(1.37 \mathrm{~g}, 45 \%)$ as a white solid.

To a mixture of lithium aluminum deuteride $(283.4 \mathrm{mg}, 6.8 \mathrm{mmol}, 1.5$ equiv) in anhydrous THF (20 mL), S-6 (1.37 g, $4.5 \mathrm{mmol}, 1$ equiv) was added slowly over 5 minutes at $0{ }^{\circ} \mathrm{C}$. Then, the reaction mixture was refluxed overnight. Then, the reaction mixture was quenched slowly with the addition of deionized $\mathrm{H}_{2} \mathrm{O}(3 \mathrm{~mL})$ at room temperature. The mixture was extracted with ethyl acetate $(3 \times 25 \mathrm{~mL})$. The combined organic layers were dried over $\mathrm{Na}_{2} \mathrm{SO}_{4}$, and filtered. The filtered solution was concentrated and the crude residue was purified by column chromatography (eluent: ethyl acetate/petroleum ether $=1: 10)$ to give $[\mathbf{D}]-\mathbf{1 b}$ as a white solid $(0.348 \mathrm{~g}, 26 \%)$. 
3.3 General procedure for the synthesis of 3 .

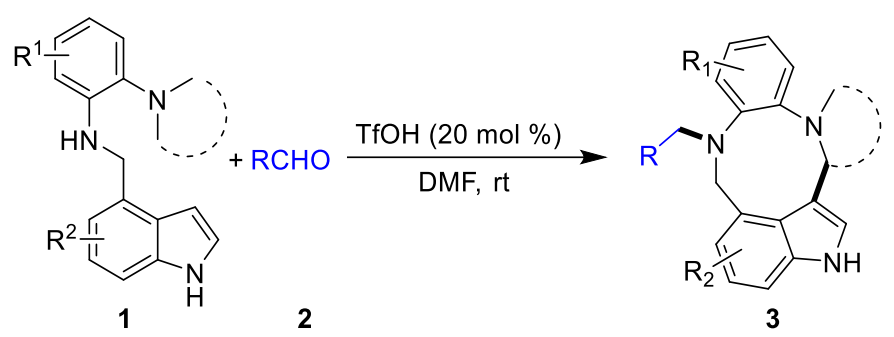

A sealed tube was charged with $1(0.2 \mathrm{mmol}), 2(0.4 \mathrm{mmol}), \mathrm{TfOH}(20 \mathrm{mmol} \%, 6.0$ $\mathrm{mg}$ ) and DMF (2.0 mL). The mixture was stirred at room temperature under an air atmosphere. After the reaction was completed (as judged by TLC analysis), the mixture was poured into a separatory funnel containing $20 \mathrm{~mL}$ of saturated $\mathrm{NaCl}$. Then, the solution was extracted by EtOAc $(30 \mathrm{~mL} \times 2)$ and the organic layers were combined. Finally, the organic layers were dried over $\mathrm{Na}_{2} \mathrm{SO}_{4}$ and concentrated under reduced pressure after filtration. The residue was directly purified by flash column chromatography on silica gel to afford the desired products 3 .

\section{Deuterium Labelling Experiments.}

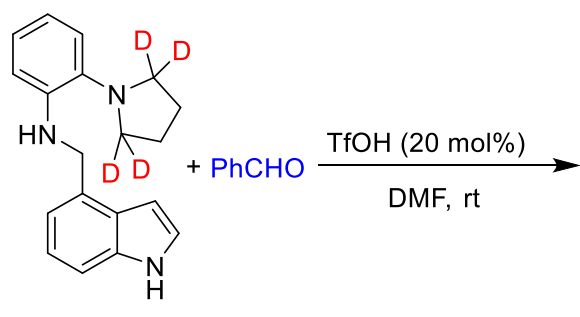

[D]-1a

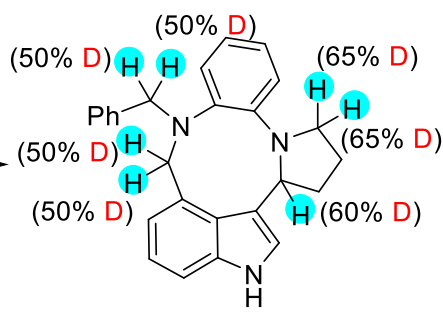

[D]-3a: $65 \%$

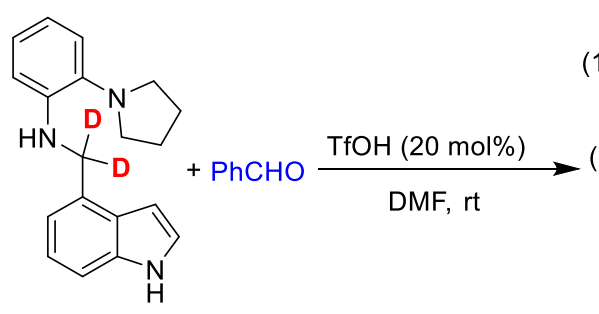

[D]-1b

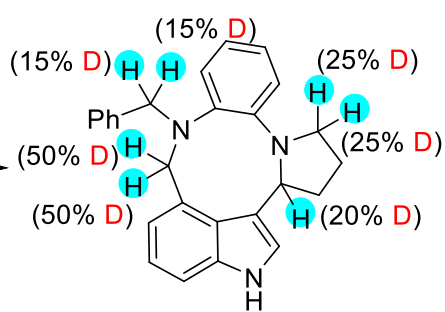

[D]-3a': $75 \%$

A sealed tube was charged with deuterated substrate [D]-1a or [D]-1b $(0.2 \mathrm{mmol}$, $)$ and PhCHO (0.6 mmol, 63.7mg), TfOH (20 mmol \%, $6.0 \mathrm{mg})$ and DMF (2.0 mL). The mixture was stirred at room temperature under an air atmosphere. After the reaction was completed (as judged by TLC analysis), the mixture was poured into a separatory funnel containing $20 \mathrm{~mL}$ of saturated $\mathrm{NaCl}$. Then, the solution was extracted by EtOAc 
$(30 \mathrm{~mL} \times 2)$ and the organic layers were combined. Finally, the organic layers were dried over $\mathrm{Na}_{2} \mathrm{SO}_{4}$ and concentrated under reduced pressure after filtration. The residue was directly purified by flash column chromatography on silica gel (eluent: ethyl acetate/petroleum ether $=1: 15)$ to afford [D]-3a (49.2 mg, 64\%) and [D]-3a' (57.3 mg, $\mathbf{7 5 \% )}$ ) as White solid.

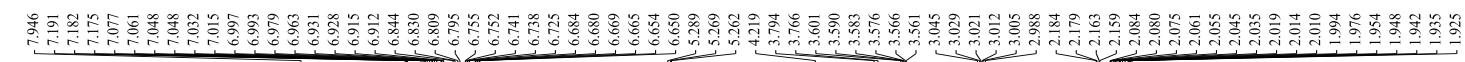

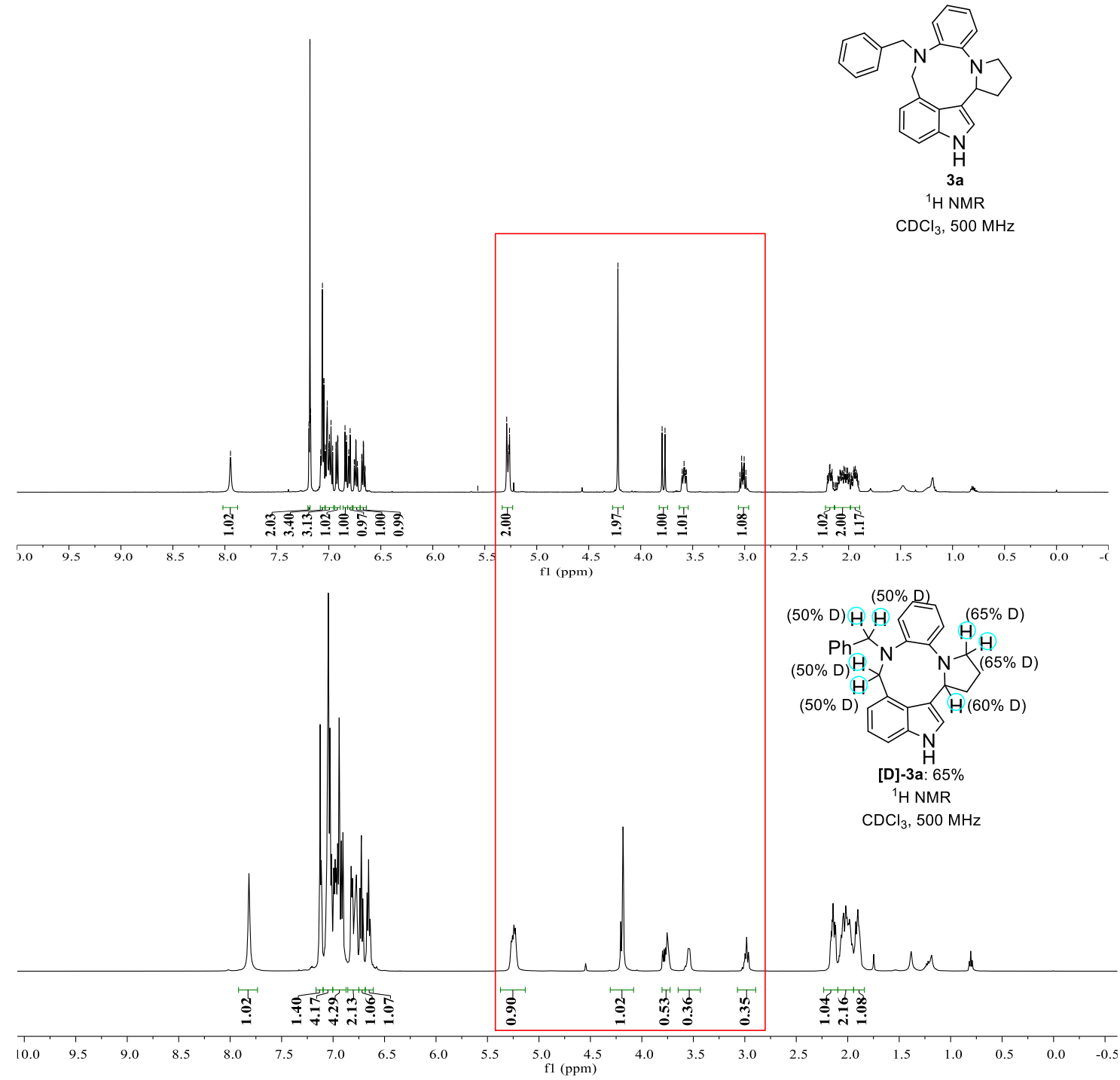




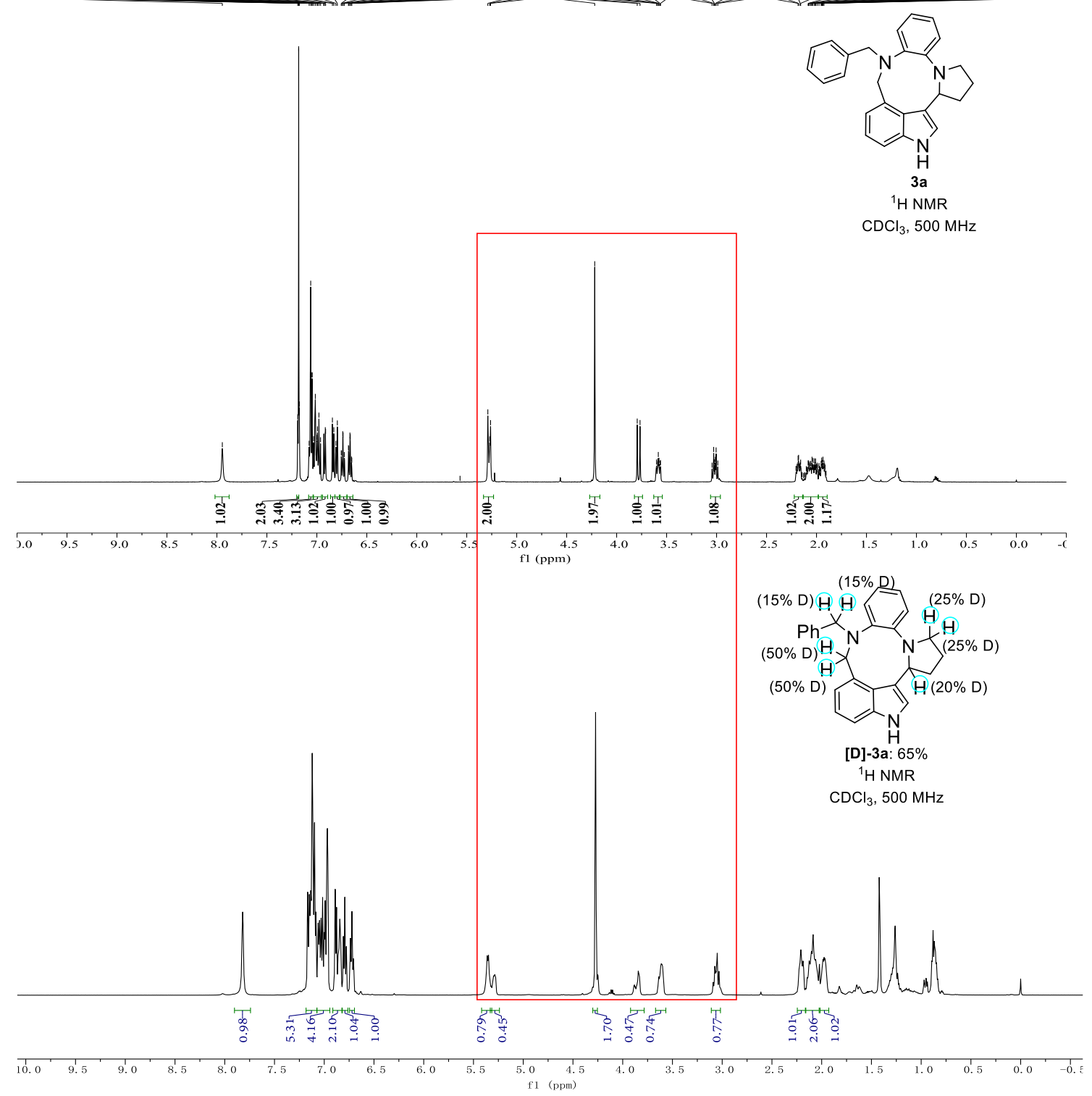

\section{Characterization of compounds 1 and 3.}<smiles>c1ccc(N2CCCC2)c(NCc2cccc3[nH]ccc23)c1</smiles>

$1 \mathrm{a}$

$N$-((1H-indol-4-yl)methyl)-2-(pyrrolidin-1-yl)aniline (1a): White solid; 1.62 g, 56\% yield; mp $92-98{ }^{\circ} \mathrm{C}$; column chromatography eluent, petroleum ether/EtOAc $=15: 1$. ${ }^{1}$ H NMR (500 MHz, CDCl $) \delta 8.26$ (s, 1H), 7.40 - 7.34 (m, 1H), 7.28 - 7.24 (m, 1H), $7.23-7.16(\mathrm{~m}, 2 \mathrm{H}), 7.07(\mathrm{~d}, J=7.6 \mathrm{~Hz}, 1 \mathrm{H}), 7.03-6.97(\mathrm{~m}, 1 \mathrm{H}), 6.80-6.65(\mathrm{~m}, 3 \mathrm{H})$, 
$5.00(\mathrm{~s}, 1 \mathrm{H}), 4.66$ (s, 2H), 3.07 (m, 4H), $1.99-1.78$ (m, 4H). ${ }^{13}$ C NMR (125 MHz, $\left.\mathbf{C D C l}_{3}\right) \delta 143.9,137.4,135.9,131.6,126.5,124.1,124.0,122.2,118.4,118.4,116.7$, 110.4, 110.1, 100.9, 51.4, 46.8, 24.1. HRMS (ESI-TOF) $\mathrm{m} / \mathrm{z}$ calcd for $\mathrm{C}_{19} \mathrm{H}_{22} \mathrm{~N}_{3}[\mathrm{M}+$ $\mathrm{H}]^{+}:$292.1808; found: 292.1808 .

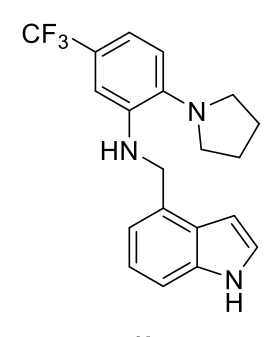

11

N-((1H-indol-4-yl)methyl)-2-(pyrrolidin-1-yl)-5-(trifluoromethyl)aniline (11) :

Brown solid; $0.65 \mathrm{~g}, 18 \%$ yield; mp $124-127{ }^{\circ} \mathrm{C}$; column chromatography eluent, petroleum ether/EtOAc $=15: 1 .{ }^{1} \mathbf{H}$ NMR $\left(\mathbf{5 0 0} \mathbf{~ M H z}, \mathbf{C D C l}_{3}\right) \delta 8.24(\mathrm{~s}, 1 \mathrm{H}), 7.40(\mathrm{~d}, J$ $=7.9 \mathrm{~Hz}, 1 \mathrm{H}), 7.28-7.26(\mathrm{~m}, 1 \mathrm{H}), 7.24(\mathrm{~d}, J=7.8 \mathrm{~Hz}, 1 \mathrm{H}), 7.21(\mathrm{~d}, J=7.1 \mathrm{~Hz}, 1 \mathrm{H})$, $7.09(\mathrm{~d}, J=7.9 \mathrm{~Hz}, 1 \mathrm{H}), 7.02(\mathrm{~d}, J=8.3 \mathrm{~Hz}, 2 \mathrm{H}), 6.73$ (t, $J=2.7 \mathrm{~Hz}, 1 \mathrm{H}), 4.87$ (s, 1H), 4.68 (s, 2H), $3.19-3.01$ (m, 4H), $2.03-1.81$ (m, 4H). ${ }^{13}$ C NMR (125 MHz, CDCl $)$ $\delta 143.3,140.6,136.0,130.7,126.7,125.4(\mathrm{q}, J=31.6 \mathrm{~Hz}), 124.2,122.2,118.8,117.4$, $113.8(\mathrm{q}, J=4.1 \mathrm{~Hz}), 110.5,106.4(\mathrm{q}, J=4.1 \mathrm{~Hz}), 100.9,50.9,46.9,24.1 .{ }^{19}$ F NMR (470 MHz, CDCl $) \delta-61.77$. HRMS (ESI-TOF) $\mathrm{m} / \mathrm{z}$ calcd for $\mathrm{C}_{20} \mathrm{H}_{21} \mathrm{~F}_{3} \mathrm{~N}_{3}[\mathrm{M}+\mathrm{H}]^{+}$: 360.1682; found: 360.1685 .

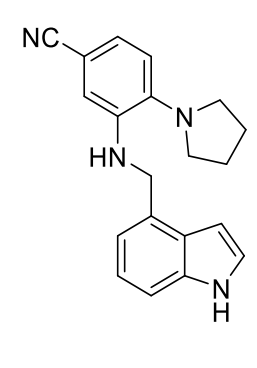

$1 \mathrm{~m}$

3-(((1H-indol-4-yl)methyl)amino)-4-(pyrrolidin-1-yl)benzonitrile (1m): White solid; $1.55 \mathrm{~g}, 49 \%$ yield; mp $157-159{ }^{\circ} \mathrm{C}$; column chromatography eluent, petroleum ether/EtOAc $=15: 1 .{ }^{1} \mathbf{H}$ NMR (500 MHz, $\left.\mathbf{C D C l}_{3}\right) \delta 8.32(\mathrm{~s}, 1 \mathrm{H}), 7.41(\mathrm{~d}, J=7.9 \mathrm{~Hz}$, 1H), 7.30 (d, $J=3.2 \mathrm{~Hz}, 1 \mathrm{H}), 7.26-7.18$ (m, 1H), 7.15 (d, J=7.2 Hz, 1H), 7.03 (dd, 
$J=8.1,1.8 \mathrm{~Hz}, 1 \mathrm{H}), 6.97(\mathrm{dd}, J=8.1,1.5 \mathrm{~Hz}, 1 \mathrm{H}), 6.90(\mathrm{~d}, J=1.7 \mathrm{~Hz}, 1 \mathrm{H}), 6.72-$ $6.64(\mathrm{~m}, 1 \mathrm{H}), 4.69(\mathrm{~s}, 1 \mathrm{H}), 4.62(\mathrm{~d}, J=4.2 \mathrm{~Hz}, 2 \mathrm{H}), 3.23-3.04(\mathrm{~m}, 4 \mathrm{H}), 1.91(\mathrm{~m}, 4 \mathrm{H})$. ${ }^{13}$ C NMR (125 MHz, CDCl 3$) \delta 142.8,142.2,136.0,130.2,126.5,124.3,122.2,121.9$, 120.6, 118.5, 117.2, 112.7, 110.6, 105.5, 100.7, 50.7, 46.8, 24.3. HRMS (ESI-TOF) $\mathrm{m} / \mathrm{z}$ calcd for $\mathrm{C}_{20} \mathrm{H}_{21} \mathrm{~N}_{4}[\mathrm{M}+\mathrm{H}]^{+}: 317.1761$; found: 317.1761 .

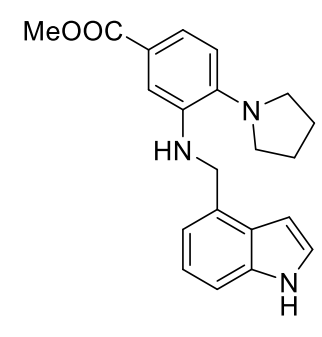

$1 \mathrm{n}$

Methyl 3-(((1H-indol-4-yl)methyl)amino)-4-(pyrrolidin-1-yl)benzoate (1n): White solid; 0.48 g, $14 \%$ yield; mp $181-184{ }^{\circ} \mathrm{C}$; column chromatography eluent, petroleum ether/EtOAc $=15: 1 .{ }^{1} \mathbf{H}$ NMR (500 MHz, $\left.\mathbf{C D C l}_{3}\right) \delta 8.42(\mathrm{~s}, 1 \mathrm{H}), 7.50(\mathrm{dd}, J=5.7,2.0$ $\mathrm{Hz}, 2 \mathrm{H}), 7.38$ (d, $J=7.9 \mathrm{~Hz}, 1 \mathrm{H}), 7.25(\mathrm{t}, J=2.9 \mathrm{~Hz}, 1 \mathrm{H}), 7.24-7.16(\mathrm{~m}, 2 \mathrm{H}), 7.01$ $(\mathrm{d}, J=8.6 \mathrm{~Hz}, 1 \mathrm{H}), 6.70(\mathrm{~s}, 1 \mathrm{H}), 4.67(\mathrm{~s}, 2 \mathrm{H}), 4.56(\mathrm{~s}, 1 \mathrm{H}), 3.92(\mathrm{~s}, 3 \mathrm{H}), 3.14(\mathrm{~d}, J=$ $6.2 \mathrm{~Hz}, 4 \mathrm{H}), 1.98$ - 1.80 (m, 4H). ${ }^{13} \mathbf{C}$ NMR (125 MHz, CDCl$) \delta 168.0,142.6,142.4$, $136.0,131.0,126.8,124.3,124.3,122.1,119.5,118.8,116.6,111.1,110.5,100.9,51.8$, 50.7, 47.1, 24.3. HRMS (ESI-TOF) $\mathrm{m} / \mathrm{z}$ calcd for $\mathrm{C}_{21} \mathrm{H}_{24} \mathrm{~N}_{3} \mathrm{O}_{2}[\mathrm{M}+\mathrm{H}]^{+}: 350.1863$; found: 350.1864 .

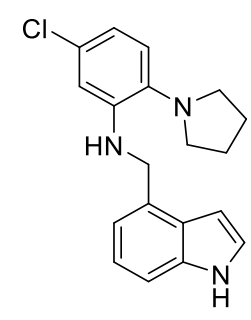

10

N-((1H-indol-4-yl)methyl)-5-chloro-2-(pyrrolidin-1-yl)aniline (10): White solid; $0.62 \mathrm{~g}, 19 \%$ yield; mp $108-112{ }^{\circ} \mathrm{C}$; column chromatography eluent, petroleum ether/EtOAc $=15: 1 .{ }^{1} \mathbf{H}$ NMR (500 MHz, $\left.\mathbf{C D C l}_{3}\right) \delta 8.28(\mathrm{~s}, 1 \mathrm{H}), 7.39(\mathrm{~d}, J=8.0 \mathrm{~Hz}$, 
1H), $7.27(\mathrm{~d}, J=3.2 \mathrm{~Hz}, 1 \mathrm{H}), 7.21(\mathrm{t}, J=7.6 \mathrm{~Hz}, 1 \mathrm{H}), 7.16(\mathrm{~d}, J=7.1 \mathrm{~Hz}, 1 \mathrm{H}), 6.95(\mathrm{~d}$, $J=8.3 \mathrm{~Hz}, 1 \mathrm{H}), 6.73-6.67(\mathrm{~m}, 2 \mathrm{H}), 6.65(\mathrm{dd}, \mathrm{J}=8.2,2.4 \mathrm{~Hz}, 1 \mathrm{H}) ., 5.04(\mathrm{~s}, 1 \mathrm{H}), 4.62$ (s, 2H), $3.10-2.92(\mathrm{~m}, 4 \mathrm{H}), 1.96-1.79(\mathrm{~m}, 4 \mathrm{H}) .{ }^{13} \mathbf{C}$ NMR (125 MHz, CDCl$) \delta$ $144.9,135.9,130.8,129.5,126.5,124.1,122.2,119.4,118.5,116.0,110.3,110.1,100.8$, 51.5, 46.6, 24.1. HRMS (ESI-TOF) $\mathrm{m} / \mathrm{z}$ calcd for $\mathrm{C}_{19} \mathrm{H}_{21} \mathrm{ClN}_{3}[\mathrm{M}+\mathrm{H}]^{+}:$326.1419; found: 326.1422 .

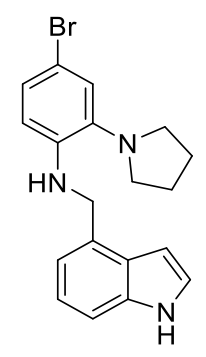

$1 p$

N-((1H-indol-4-yl)methyl)-4-bromo-2-(pyrrolidin-1-yl)aniline (1p): Light yellow solid; $1.25 \mathrm{~g}, 34 \%$ yield; mp $95-100{ }^{\circ} \mathrm{C}$; column chromatography eluent, petroleum ether/EtOAc $=15: 1 .{ }^{1} \mathbf{H}$ NMR (500 MHz, $\left.\mathbf{C D C l}_{3}\right) \delta 8.26(\mathrm{~s}, 1 \mathrm{H}), 7.37(\mathrm{~d}, J=8.0 \mathrm{~Hz}$, 1H), $7.26(\mathrm{t}, J=2.9 \mathrm{~Hz}, 1 \mathrm{H}), 7.21(\mathrm{t}, J=7.6 \mathrm{~Hz}, 1 \mathrm{H}), 7.18-7.12(\mathrm{~m}, 2 \mathrm{H}), 7.08(\mathrm{dd}, J$ $=8.5,2.3 \mathrm{~Hz}, 1 \mathrm{H}), 6.73-6.65(\mathrm{~m}, 1 \mathrm{H}), 6.59(\mathrm{~d}, J=8.5 \mathrm{~Hz}, 1 \mathrm{H}), 4.91(\mathrm{~s}, 1 \mathrm{H}), 4.64(\mathrm{~s}$, 2H), 3.07 (t, $J=6.1 \mathrm{~Hz}, 4 \mathrm{H}), 1.97-1.83(\mathrm{~m}, 4 \mathrm{H}) .{ }^{13} \mathbf{C}$ NMR (125 MHz, CDCl $) \delta$ 142.8, 138.9, 135.9, 131.0, 126.5, 126.4, 124.2, 122.2 121.5, 118.3, 111.6, 110.3, 108.4, 100.7, 51.3, 46.7, 24.1. HRMS (ESI-TOF) $\mathrm{m} / \mathrm{z}$ calcd for $\mathrm{C}_{19} \mathrm{H}_{21} \mathrm{BrN}_{3}[\mathrm{M}+\mathrm{H}]^{+}$: 370.0913; found: 370.0916 .<smiles>Cc1ccc(NCc2cccc3[nH]ccc23)c(N2CCCC2)c1</smiles>

N-((1H-indol-4-yl)methyl)-4-methyl-2-(pyrrolidin-1-yl)aniline (1q): White solid; $0.98 \mathrm{~g}, 32 \%$ yield; mp $122-124{ }^{\circ} \mathrm{C}$; column chromatography eluent, petroleum 
ether/EtOAc $=15: 1 .{ }^{1} \mathbf{H}$ NMR (500 MHz, $\left.\mathbf{C D C l}_{3}\right) \delta 8.13(\mathrm{~s}, 1 \mathrm{H}), 7.23(\mathrm{q}, J=4.0,3.4$ $\mathrm{Hz}, 1 \mathrm{H}), 7.12$ (t, $J=2.9 \mathrm{~Hz}, 1 \mathrm{H}), 7.08$ (d, $J=5.1 \mathrm{~Hz}, 2 \mathrm{H}), 6.79$ (d, $J=2.0 \mathrm{~Hz}, 1 \mathrm{H})$, $6.70(\mathrm{dd}, J=8.0,2.0 \mathrm{~Hz}, 1 \mathrm{H}), 6.62-6.57(\mathrm{~m}, 1 \mathrm{H}), 6.55(\mathrm{~d}, J=8.0 \mathrm{~Hz}, 1 \mathrm{H}), 4.71(\mathrm{~s}$, 1H), $4.53(\mathrm{~s}, 2 \mathrm{H}), 3.04-2.90(\mathrm{~m}, 4 \mathrm{H}), 2.18(\mathrm{~s}, 3 \mathrm{H}), 1.81-1.74(\mathrm{~m}, 4 \mathrm{H}) .{ }^{13} \mathrm{C}$ NMR (125 MHz, $\left.\mathbf{C D C l}_{3}\right) \delta 141.5,137.4,135.9,131.8,126.5,125.9,124.2,124.0,122.2$, 119.2, 118.3, 110.5, 110.0, 100.9, 51.3, 47.1, 24.1, 20.8. HRMS (ESI-TOF) m/z calcd for $\mathrm{C}_{20} \mathrm{H}_{24} \mathrm{~N}_{3}[\mathrm{M}+\mathrm{H}]^{+}$: 306.1965; found: 306.1967 .<smiles>COc1ccc(N2CCCC2)c(NCc2cccc3[nH]ccc23)c1</smiles>

N-((1H-indol-4-yl)methyl)-5-methoxy-2-(pyrrolidin-1-yl)aniline (1r): White solid; $0.71 \mathrm{~g}, 22 \%$ yield; mp $98-104{ }^{\circ} \mathrm{C}$; column chromatography eluent, petroleum ether/EtOAc $=15: 1 .{ }^{1} \mathbf{H}$ NMR (500 MHz, $\left.\mathbf{C D C l}_{3}\right) \delta 8.13(\mathrm{~s}, 1 \mathrm{H}), 7.21(\mathrm{dt}, J=6.4,3.3$ $\mathrm{Hz}, 1 \mathrm{H}), 7.11-7.08$ (m, 1H), $7.08-7.05$ (m, 2H), 6.90 (d, J=8.5 Hz, 1H), $6.60-6.53$ (m, 1H), $6.24(\mathrm{~d}, J=2.8 \mathrm{~Hz}, 1 \mathrm{H}), 6.13(\mathrm{dd}, J=8.5,2.8 \mathrm{~Hz}, 1 \mathrm{H}), 5.14(\mathrm{~s}, 1 \mathrm{H}), 4.53(\mathrm{~s}$, 2H), 3.64 (s, 3H), $2.98-2.75$ (m, 4H), $1.83-1.68$ (m, 4H). ${ }^{13}$ C NMR (125 MHz, $\left.\mathbf{C D C l}_{3}\right) \delta 167.9,142.5,142.4,136.0,131.1,126.8,124.4,124.2,122.1,119.5,118.9$, 116.6, 111.1, 110.4, 101.0, 51.8, 50.7, 47.1, 24.3. HRMS (ESI-TOF) m/z calcd for $\mathrm{C}_{20} \mathrm{H}_{24} \mathrm{~N}_{3} \mathrm{O}[\mathrm{M}+\mathrm{H}]^{+}:$322.1914; found: 322.1915 .

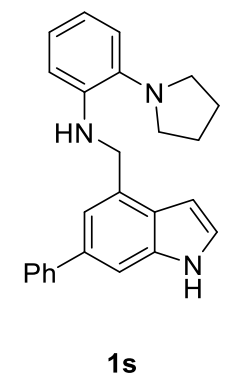


N-((6-phenyl-1H-indol-4-yl)methyl)-2-(pyrrolidin-1-yl)aniline(1s): White solid; $0.79 \mathrm{~g}, 22 \%$ yield; mp $92-94{ }^{\circ} \mathrm{C}$; column chromatography eluent, petroleum ether/EtOAc $=15: 1 .{ }^{1} \mathbf{H}$ NMR (500 MHz, $\left.\mathbf{C D C l}_{3}\right) \delta 8.01(\mathrm{~s}, 1 \mathrm{H}), 7.53-7.42(\mathrm{~m}, 2 \mathrm{H})$, $7.31-7.24(\mathrm{~m}, 3 \mathrm{H}), 7.22-7.14(\mathrm{~m}, 1 \mathrm{H}), 6.98(\mathrm{t}, J=2.8 \mathrm{~Hz}, 1 \mathrm{H}), 6.95(\mathrm{dd}, J=7.8,1.2$ $\mathrm{Hz}, 1 \mathrm{H}), 6.91-6.85(\mathrm{~m}, 1 \mathrm{H}), 6.71-6.56(\mathrm{~m}, 3 \mathrm{H}), 6.55-6.50(\mathrm{~m}, 1 \mathrm{H}), 4.89(\mathrm{~s}, 1 \mathrm{H})$, 4.55 (s, 2H), 2.93 (d, $J=3.4 \mathrm{~Hz}, 4 \mathrm{H}), 1.77-1.65$ (m, 4H). ${ }^{13} \mathbf{C}$ NMR (125 MHz, CDCl 3$)$ $\delta 144.0,142.3,137.6,136.7,135.6,131.9,128.9,126.8,126.1,125.0,124.3,118.6$, 118.5, 117.1, 115.7, 110.7, 108.8, 100.8, 51.5, 47.3, 24.2. HRMS (ESI-TOF) m/z calcd for $\mathrm{C}_{26} \mathrm{H}_{25} \mathrm{~N}_{3}[\mathrm{M}+\mathrm{H}]^{+}$: 368.2121; found: 368.2125 .

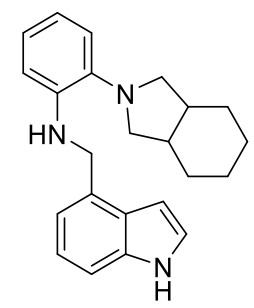

1t

N-((1H-indol-4-yl)methyl)-2-(octahydro-2H-isoindol-2-yl)aniline (1t):Light yellow solid; 1.98 g, 57\% yield; mp $88-92{ }^{\circ} \mathrm{C}$; column chromatography eluent, petroleum ether/EtOAc $=15: 1 .{ }^{1} \mathbf{H}$ NMR $\left(\mathbf{5 0 0} \mathbf{~ M H z}, \mathbf{C D C l}_{3}\right) \delta 8.25(\mathrm{~s}, 1 \mathrm{H}), 7.36(\mathrm{dd}, J=6.6,2.4$ $\mathrm{Hz}, 1 \mathrm{H}), 7.27-7.20(\mathrm{~m}, 3 \mathrm{H}), 7.14(\mathrm{~d}, J=7.7 \mathrm{~Hz}, 1 \mathrm{H}), 7.04-6.96(\mathrm{~m}, 1 \mathrm{H}), 6.83-6.70$ $(\mathrm{m}, 3 \mathrm{H}), 4.82(\mathrm{~s}, 1 \mathrm{H}), 4.70(\mathrm{~s}, 2 \mathrm{H}), 3.28(\mathrm{dd}, J=9.1,6.5 \mathrm{~Hz}, 2 \mathrm{H}), 3.14(\mathrm{dd}, J=9.2,5.0$ $\mathrm{Hz}, 2 \mathrm{H}), 2.40-2.22(\mathrm{~m}, 2 \mathrm{H}), 1.68-1.51(\mathrm{~m}, 6 \mathrm{H}), 1.40(\mathrm{dd}, J=9.3,4.9 \mathrm{~Hz}, 2 \mathrm{H}) .{ }^{13} \mathrm{C}$ NMR (125 MHz, $\left.\mathbf{C D C l}_{3}\right) \delta$ 143.7, 138.9, 135.9, 131.7, 126.4, 124.0, 123.2, 122.2, $118.3,118.2,117.0,110.7,110.1,100.9,55.7,47.0,37.6,26.7,23.1$. HRMS (ESITOF) $\mathrm{m} / \mathrm{z}$ calcd for $\mathrm{C}_{23} \mathrm{H}_{28} \mathrm{~N}_{3}[\mathrm{M}+\mathrm{H}]^{+}: 346.2278$; found: 346.2277 .

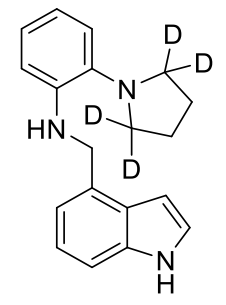

[D]-1a 
N-((1H-indol-4-yl)methyl)-2-(pyrrolidin-1-yl-2,2,5,5-d4)aniline(D-1a): yellow oil; $404 \mathrm{mg}, 57 \%$ yield; column chromatography eluent, petroleum ether/EtOAc $=15: 1 .{ }^{1} \mathbf{H}$ NMR (500 MHz, CDCl $) \delta 8.24(\mathrm{~s}, 1 \mathrm{H}), 7.08(\mathrm{dd}, J=7.7,1.4 \mathrm{~Hz}, 1 \mathrm{H}), 7.01(\mathrm{td}, J=$ 7.8, 1.4 Hz, 1H), $6.79-6.73(\mathrm{~m}, 2 \mathrm{H}), 6.72(\mathrm{~m}, 1 \mathrm{H}), 5.00(\mathrm{~s}, 1 \mathrm{H}), 4.68(\mathrm{~s}, 2 \mathrm{H}), 1.89$ (s, 4H). ${ }^{13} \mathbf{C}$ NMR (125 MHz, CDCl $) \delta 143.88,137.43,135.89,131.62,126.52,124.04$, 124.02, 122.16, 118.39, 118.33, 116.69, 110.35, 110.09, 100.90, 50.61 (m), 46.82, 23.90. HRMS(ESI-TOF) $\mathrm{m} / \mathrm{z}$ calcd for $\mathrm{C}_{19} \mathrm{H}_{18} \mathrm{D}_{4} \mathrm{~N}_{3}[\mathrm{M}+\mathrm{H}]^{+}$: 296.2059; found: 296.2059.

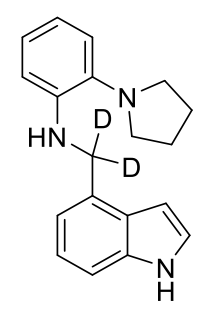

[D]-1b

N-((1H-indol-4-yl)methyl-d2)-2-(pyrrolidin-1-yl)aniline: According to the references for two steps, indole-4-carboxylic acid (403 mg, $2.5 \mathrm{mmol})$, afforded [D]1b $(20.5 \mathrm{mg}, 2.8 \%)$ as a yellow oil after purification on silica gel (petroleum ether/EtOAc = 10:1). ${ }^{\mathbf{1}} \mathbf{H}$ NMR (500 MHz, $\left.\mathbf{C D C l}_{3}\right) \delta 7.95(\mathrm{~s}, 1 \mathrm{H}), 7.12(\mathrm{dd}, J=6.4,2.8$ Hz, 1H), 7.05 (s, 1H), 6.98 (s, 1H), 6.95 (d, J= 7.7 Hz, 1H), 6.87 (t, J= 7.7 Hz, 1H), $6.64-6.58(\mathrm{~m}, 2 \mathrm{H}), 6.53(\mathrm{~s}, 1 \mathrm{H}), 4.86(\mathrm{~s}, 1 \mathrm{H}), 2.92(\mathrm{~s}, 3 \mathrm{H}), 1.73$ (s, 2H). ${ }^{13} \mathbf{C}$ NMR (126 MHz, CDCl $) \delta 144.07,137.53,136.01,131.53,126.64,124.26,124.20,122.17$, $118.62,118.47,116.87,110.56,110.27,100.80,51.50,46.30$ (m), 24.24. HRMS(ESITOF) $\mathrm{m} / \mathrm{z}$ calcd for $\mathrm{C}_{19} \mathrm{H}_{20} \mathrm{D}_{2} \mathrm{~N}_{3}{ }^{+}[\mathrm{M}+\mathrm{H}]^{+}$: 294.1934; found: 294.1935 .

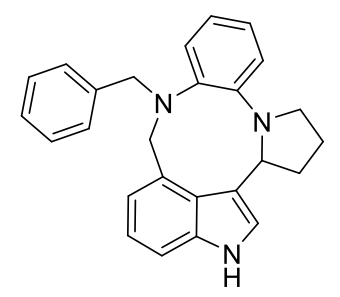

$3 a$ 


\section{7-benzyl-6,7,13,14,15,15a-hexahydro-2H-benzo[2,3]pyrrolo[1',2':4,5][1,4] diazo-}

nino[6,7,8-cd]indole (3a): According to general procedure (for $36 \mathrm{~h}), \mathbf{1 a}(58.4 \mathrm{mg}, 0.2$ mmol), 2a (42.4 mg, $0.4 \mathrm{mmol}), \mathrm{TfOH}(6.0 \mathrm{mg}, 0.04 \mathrm{mmol})$, afforded $\mathbf{3 a}(67.1 \mathrm{mg}$, $89 \%$ ) as a white solid after purification on silica gel (petroleum ether/EtOAc $=15: 1$ ), mp $140-144{ }^{\circ} \mathrm{C}$. For Scale-up Experiment, an 50-mL sealed tube equipped with and magnetic stir bar was charged with 1a $(1.46 \mathrm{~g}, 5.0 \mathrm{mmol}), \mathbf{2 a}(1.06 \mathrm{~g}, 10.0 \mathrm{mmol})$, and TfOH (150.1 mg, $1 \mathrm{mmol})$. DMF (20.0 mL) were then added with syringe. The mixture was stirred under room temperature for $72 \mathrm{~h}$, affording $\mathbf{3 a}$ with $73 \%$ yield $(1.38 \mathrm{~g})$.

${ }^{1}$ H NMR (500 MHz, CDCl $) \delta 7.95(\mathrm{~s}, 1 \mathrm{H}), 7.19(\mathrm{~d}, J=4.6 \mathrm{~Hz}, 2 \mathrm{H}), 7.08-7.04(\mathrm{~m}$, 3H), $7.03-6.95(\mathrm{~m}, 3 \mathrm{H}), 6.92(\mathrm{dd}, J=7.8,1.6 \mathrm{~Hz}, 1 \mathrm{H}), 6.84(\mathrm{~d}, J=7.1 \mathrm{~Hz}, 1 \mathrm{H}), 6.80$ $(\mathrm{d}, J=7.2 \mathrm{~Hz}, 1 \mathrm{H}), 6.77-6.70(\mathrm{~m}, 1 \mathrm{H}), 6.67(\mathrm{td}, J=7.5,1.5 \mathrm{~Hz}, 1 \mathrm{H}), 5.33-5.24(\mathrm{~m}$, 2H), $4.22(\mathrm{~s}, 2 \mathrm{H}), 3.78(\mathrm{~d}, J=14.1 \mathrm{~Hz}, 1 \mathrm{H}), 3.62-3.54(\mathrm{~m}, 1 \mathrm{H}), 3.02(\mathrm{dt}, J=12.1,8.3$ Hz, 1H), $2.22-2.14$ (m, 1H), $2.13-1.98$ (m, 2H), $1.98-1.89$ (m, 1H). ${ }^{13}$ C NMR (125 $\left.\mathbf{M H z}_{\mathbf{1}} \mathbf{C D C l}_{3}\right) \delta 146.0,142.7,139.1,138.1,134.6,128.9,127.9,127.3,126.5,121.9$, $121.4,121.3,120.8,120.6,120.2$, 120.1, 116.9, 110.4, 61.1, 59.4, 55.0, 46.1, 28.5, 24.6. HRMS (ESI-TOF) $\mathrm{m} / \mathrm{z}$ calcd for $\mathrm{C}_{26} \mathrm{H}_{26} \mathrm{~N}_{3}[\mathrm{M}+\mathrm{H}]^{+}: 380.2121$; found: 380.2124 .

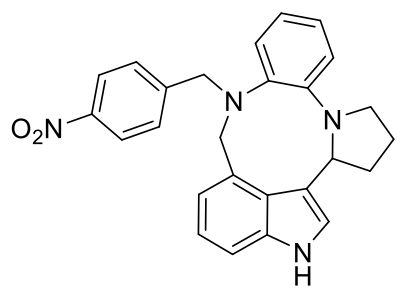

$3 \mathbf{b}$

7-(4-nitrobenzyl)-6,7,13,14,15,15a-hexahydro-2H-benzo[2,3]pyrrolo[1',2':4,5][1,4] diazonino[6,7,8-cd]indole (3b): According to general procedure (for $36 \mathrm{~h}$ ), 1a (58.4 mg, $0.2 \mathrm{mmol}), 2 \mathbf{b}$ (60.4 mg, $0.4 \mathrm{mmol})$, TfOH (6.0 mg, $0.04 \mathrm{mmol})$, afforded $\mathbf{3 b}$ (69.0 $\mathrm{mg}, 82 \%$ ) as a light yellow solid after purification on silica gel (petroleum ether/EtOAc $=15: 1), \operatorname{mp} 80-84{ }^{\circ} \mathrm{C} .{ }^{1} \mathbf{H}$ NMR (500 MHz, CDCl 3$) \delta 8.01(\mathrm{~s}, 1 \mathrm{H}), 7.85(\mathrm{~d}, J=8.7$ $\mathrm{Hz}, 2 \mathrm{H}), 7.20-7.17(\mathrm{~m}, 2 \mathrm{H}), 7.16(\mathrm{~s}, 1 \mathrm{H}), 7.05-7.00(\mathrm{~m}, 1 \mathrm{H}), 6.99-6.92(\mathrm{~m}, 1 \mathrm{H})$, $6.88-6.73(\mathrm{~m}, 4 \mathrm{H}), 6.68(\mathrm{td}, J=7.6,1.4 \mathrm{~Hz}, 1 \mathrm{H}), 5.32(\mathrm{~d}, J=14.0 \mathrm{~Hz}, 1 \mathrm{H}), 5.09(\mathrm{~d}, J$ $=7.1 \mathrm{~Hz}, 1 \mathrm{H}), 4.34(\mathrm{~d}, J=14.8 \mathrm{~Hz}, 1 \mathrm{H}), 4.25(\mathrm{~d}, J=14.8 \mathrm{~Hz}, 1 \mathrm{H}), 3.75(\mathrm{~d}, J=14.1$ Hz, 1H), 3.57 (ddd, $J=11.5,7.6,2.9 \mathrm{~Hz}, 1 \mathrm{H}), 3.01$ (dt, $J=12.2,8.2 \mathrm{~Hz}, 1 \mathrm{H}), 2.24-$ 
2.15 (m, 1H), $2.11-1.97(\mathrm{~m}, 2 \mathrm{H}), 1.97-1.89(\mathrm{~m}, 1 \mathrm{H}) .{ }^{13} \mathbf{C} \mathbf{N M R}\left(\mathbf{1 2 5} \mathbf{M H z}, \mathbf{C D C l}_{\mathbf{3}}\right)$ $\delta 147.0,146.7,146.0,142.2,138.1,134.0,129.6,127.1,123.2,122.4,121.6,121.5$, $120.8,120.4,120.3,119.4,117.3,110.8,61.7,59.9,54.4,46.1,28.5,24.6$. HRMS (ESITOF) $\mathrm{m} / \mathrm{z}$ calcd for $\mathrm{C}_{26} \mathrm{H}_{25} \mathrm{~N}_{4} \mathrm{O}_{2}[\mathrm{M}+\mathrm{H}]^{+}$: 425.1972; found: 425.1976 .

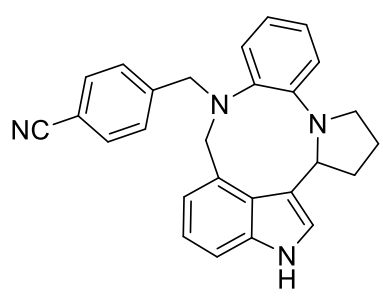

$3 c$

\section{4-((2,6,13,14,15,15a-hexahydro-7H-benzo[2,3]pyrrolo[1',2':4,5][1,4]diazonino $[6,7$,} 8-cd]indol-7-yl)methyl)benzonitrile (3c): According to general procedure (for $36 \mathrm{~h}$ ), 1a (58.4 mg, $0.2 \mathrm{mmol}), 2 \mathrm{c}(52.5 \mathrm{mg}, 0.4 \mathrm{mmol}), \mathrm{TfOH}(6.0 \mathrm{mg}, 0.04 \mathrm{mmol})$, afforded 3c $(64.0 \mathrm{mg}, 79 \%)$ as a white solid after purification on silica gel (petroleum ether/EtOAc = 15:1), mp $95-100{ }^{\circ} \mathrm{C} .{ }^{\mathbf{1}} \mathbf{H}$ NMR (500 MHz, CDCl$) \delta 7.98(\mathrm{~s}, 1 \mathrm{H})$, $7.26(\mathrm{~d}, J=8.3 \mathrm{~Hz}, 2 \mathrm{H}), 7.16(\mathrm{~d}, J=9.1 \mathrm{~Hz}, 1 \mathrm{H}), 7.10(\mathrm{~d}, J=8.2 \mathrm{~Hz}, 2 \mathrm{H}), 7.01-6.91$ (m, 2H), $6.86-6.72(\mathrm{~m}, 4 \mathrm{H}), 6.67(\mathrm{td}, J=7.5,1.6 \mathrm{~Hz}, 1 \mathrm{H}), 5.29(\mathrm{~d}, J=14.0 \mathrm{~Hz}, 1 \mathrm{H})$, $5.08(\mathrm{~d}, J=7.1 \mathrm{~Hz}, 1 \mathrm{H}), 4.27$ (d, $J=14.7 \mathrm{~Hz}, 1 \mathrm{H}), 4.20$ (d, $J=14.7 \mathrm{~Hz}, 1 \mathrm{H}), 3.73$ (d, $J=14.0 \mathrm{~Hz}, 1 \mathrm{H}), 3.56(\mathrm{ddd}, J=11.4,7.6,2.9 \mathrm{~Hz}, 1 \mathrm{H}), 2.99$ (dt, $J=12.3,8.2 \mathrm{~Hz}, 1 \mathrm{H})$, $2.23-2.12(\mathrm{~m}, 1 \mathrm{H}), 2.10-1.96(\mathrm{~m}, 2 \mathrm{H}), 1.96-1.86$ (m, 1H). ${ }^{13} \mathrm{C}$ NMR (125 MHz, $\left.\mathbf{C D C l}_{3}\right) \delta 146.0,144.8,142.3,138.1,134.1,131.8,129.5,127.1,122.4,121.5,121.5$, $120.8,120.4$, 120.2 119.5, 119.1, 117.3, 110.8, 110.3, 61.6, 59.8, 54.8, 46.2, 28.6, 24.6. HRMS (ESI-TOF) $\mathrm{m} / \mathrm{z}$ calcd for $\mathrm{C}_{27} \mathrm{H}_{25} \mathrm{~N}_{4}[\mathrm{M}+\mathrm{H}]^{+}$: 405.2074; found: 405.2077 .

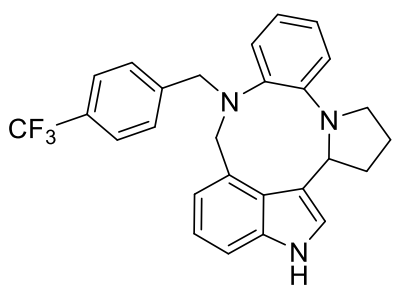

$3 d$

7-(4-(trifluoromethyl)benzyl)-6,7,13,14,15,15a-hexahydro-2H-benzo[2,3]Pyrrolo $\left[1^{\prime}, 2^{\prime}: 4,5\right][1,4]$ diazonino[6,7,8-cd]indole (3d): According to general procedure (for 36 h), 1a (58.4 mg, $0.2 \mathrm{mmol}), 2 d(69.8 \mathrm{mg}, 0.4 \mathrm{mmol}), \mathrm{TfOH}(6.0 \mathrm{mg}, 0.04 \mathrm{mmol})$, 
afforded $\mathbf{3 d}(71.8 \mathrm{mg}, 80 \%)$ as a white solid after purification on silica gel (petroleum ether/EtOAc = 15:1), mp $68-72{ }^{\circ} \mathrm{C}^{1}{ }^{1} \mathbf{H}$ NMR (500 $\left.\mathbf{~ M H z , ~} \mathbf{C D C l}_{3}\right) \delta 7.98(\mathrm{~s}, 1 \mathrm{H}), 7.27$ $(\mathrm{d}, J=7.8 \mathrm{~Hz}, 2 \mathrm{H}), 7.19(\mathrm{~d}, J=5.6 \mathrm{~Hz}, 1 \mathrm{H}), 7.15(\mathrm{~d}, J=7.8 \mathrm{~Hz}, 2 \mathrm{H}), 7.03(\mathrm{~s}, 1 \mathrm{H}), 7.00$ - $6.94(\mathrm{~m}, 1 \mathrm{H}), 6.91-6.85(\mathrm{~m}, 1 \mathrm{H}), 6.82$ (t, $J=7.1 \mathrm{~Hz}, 2 \mathrm{H}), 6.77$ (t, $J=7.5 \mathrm{~Hz}, 1 \mathrm{H})$, $6.69(\mathrm{~d}, J=7.5 \mathrm{~Hz}, 1 \mathrm{H}), 5.30(\mathrm{t}, J=12.0 \mathrm{~Hz}, 1 \mathrm{H}), 5.15(\mathrm{~d}, J=5.8 \mathrm{~Hz}, 1 \mathrm{H}), 4.26(\mathrm{q}, J$ $=14.6 \mathrm{~Hz}, 2 \mathrm{H}), 3.76(\mathrm{~d}, J=14.0 \mathrm{~Hz}, 1 \mathrm{H}), 3.63-3.54(\mathrm{~m}, 1 \mathrm{H}), 3.02(\mathrm{dt}, J=11.8,8.3$ Hz, 1H), $2.24-2.12(\mathrm{~m}, 1 \mathrm{H}), 2.12-1.98(\mathrm{~m}, 2 \mathrm{H}), 1.98-1.88(\mathrm{~m}, 1 \mathrm{H}) .{ }^{13} \mathbf{C}$ NMR (125 MHz, CDCl $) \delta 146.0,143.2,142.4,138.1,134.2,129.0,128.7(\mathrm{q}, J=32.1 \mathrm{~Hz}), 127.2$, 124.8(q, $J=3.8 \mathrm{~Hz}), 122.2,121.5,121.4,120.8,120.4,120.4,119.6,117.1,110.6,61.5$,

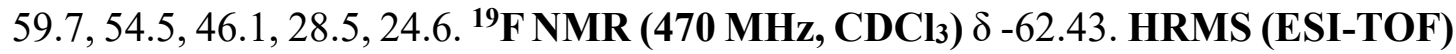
$\mathrm{m} / \mathrm{z}$ calcd for $\mathrm{C}_{27} \mathrm{H}_{25} \mathrm{~F}_{3} \mathrm{~N}_{3}[\mathrm{M}+\mathrm{H}]^{+}$: 448.1995; found: 448.1994 .

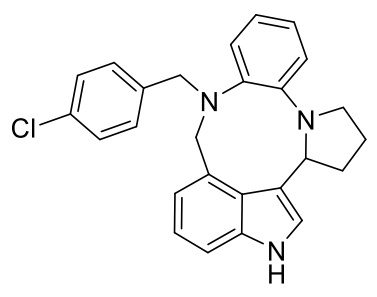

$3 e$

7-(4-chlorobenzyl)-6,7,13,14,15,15a-hexahydro-2H-benzo[2,3]pyrrolo[1',2':4,5][1, 4]diazonino[6,7,8-cd]indole (3e): According to general procedure (for $36 \mathrm{~h}$ ), $\mathbf{1 a}(58.4$ mg, $0.2 \mathrm{mmol}), \mathbf{2 e}(56.3 \mathrm{mg}, 0.4 \mathrm{mmol})$, TfOH (6.0 mg, $0.04 \mathrm{mmol})$, afforded $\mathbf{3 e}$ (48.4 $\mathrm{mg}, 59 \%)$ as a white solid after purification on silica gel (petroleum ether/EtOAc $=$ 15:1), mp 68 - $72{ }^{\circ} \mathrm{C} .{ }^{1} \mathbf{H}$ NMR (500 MHz, $\left.\mathbf{C D C l}_{3}\right) \delta 7.94$ (s, 1H), 7.18 (s, 1H), $7.06-$ $6.89(\mathrm{~m}, 6 \mathrm{H}), 6.87(\mathrm{~d}, J=7.8 \mathrm{~Hz}, 1 \mathrm{H}), 6.84-6.76(\mathrm{~m}, 2 \mathrm{H}), 6.76-6.70(\mathrm{~m}, 1 \mathrm{H}), 6.67$ $(\mathrm{t}, J=7.5 \mathrm{~Hz}, 1 \mathrm{H}), 5.25(\mathrm{~d}, J=14.0 \mathrm{~Hz}, 1 \mathrm{H}), 5.16(\mathrm{~d}, J=6.9 \mathrm{~Hz}, 1 \mathrm{H}), 4.26-4.07(\mathrm{~m}$, 2H), $3.75(\mathrm{~d}, J=14.0 \mathrm{~Hz}, 1 \mathrm{H}), 3.62-3.49$ (m, 1H), 3.00 (dt, $J=12.1,8.3 \mathrm{~Hz}, 1 \mathrm{H})$, $2.22-2.11(\mathrm{~m}, 1 \mathrm{H}), 2.10-1.96(\mathrm{~m}, 2 \mathrm{H}), 1.96-1.87(\mathrm{~m}, 1 \mathrm{H}) .{ }^{13} \mathbf{C}$ NMR (125 MHz, $\left.\mathbf{C D C l}_{3}\right) \delta 153.0,146.2,142.4,141.4,138.0,134.1,127.2,122.5,121.4,121.2,120.9$, $120.7,120.4,120.1,116.8,110.5,110.1,108.4,60.7,57.6,47.5,46.1,28.4,24.5$. HRMS (ESI-TOF) m/z calcd for $\mathrm{C}_{26} \mathrm{H}_{25} \mathrm{ClN}_{3}[\mathrm{M}+\mathrm{H}]^{+}: 414.1732$; found: 414.1740 . 


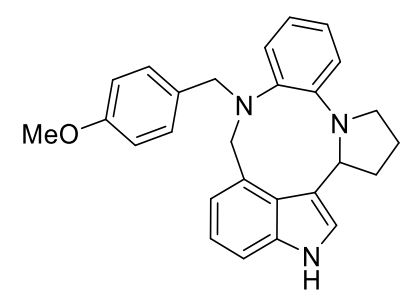

$3 f$

7-(4-methoxybenzyl)-6,7,13,14,15,15a-hexahydro-2H-benzo[2,3]pyrrolo[1',2':4,5] [1,4]diazonino[6,7,8-cd]indole (3f): According to general procedure (for $36 \mathrm{~h}$ ), 1a (58.4 mg, $0.2 \mathrm{mmol}), \mathbf{2 f}(54.5 \mathrm{mg}, 0.4 \mathrm{mmol})$, TfOH (6.0 mg, $0.04 \mathrm{mmol})$, afforded $\mathbf{3 f}$ $(55.8 \mathrm{mg}, 68 \%)$ as a white solid after purification on silica gel (petroleum ether/EtOAc $=15: 1), \operatorname{mp} 96-100{ }^{\circ} \mathrm{C} .{ }^{1} \mathbf{H}$ NMR (500 MHz, $\left.\mathbf{C D C l}_{3}\right) \delta 7.91(\mathrm{~s}, 1 \mathrm{H}), 7.15(\mathrm{~s}, 1 \mathrm{H})$, $7.04-6.94(\mathrm{~m}, 4 \mathrm{H}), 6.92(\mathrm{dd}, J=7.8,1.6 \mathrm{~Hz}, 1 \mathrm{H}), 6.83(\mathrm{~d}, J=7.1 \mathrm{~Hz}, 1 \mathrm{H}), 6.78(\mathrm{dd}$, $J=7.9,1.6 \mathrm{~Hz}, 1 \mathrm{H}), 6.73(\mathrm{td}, J=7.6,1.6 \mathrm{~Hz}, 1 \mathrm{H}), 6.67(\mathrm{td}, J=7.4,1.6 \mathrm{~Hz}, 1 \mathrm{H}), 6.58$ $(\mathrm{d}, J=8.6 \mathrm{~Hz}, 2 \mathrm{H}), 5.27(\mathrm{~d}, J=7.0 \mathrm{~Hz}, 1 \mathrm{H}), 5.21(\mathrm{~d}, J=14.0 \mathrm{~Hz}, 1 \mathrm{H}), 4.14(\mathrm{~s}, 2 \mathrm{H})$, $3.77(\mathrm{~d}, J=14.1 \mathrm{~Hz}, 1 \mathrm{H}), 3.61(\mathrm{~s}, 3 \mathrm{H}), 3.56(\mathrm{td}, J=8.2,7.8,3.9 \mathrm{~Hz}, 1 \mathrm{H}), 3.00(\mathrm{dt}, J=$ 12.1, 8.2 Hz, 1H), $2.21-2.12(\mathrm{~m}, 1 \mathrm{H}), 2.10-1.97(\mathrm{~m}, 2 \mathrm{H}), 1.96-1.87(\mathrm{~m}, 1 \mathrm{H}) .{ }^{13} \mathrm{C}$ NMR (125 MHz, CDCl 3$) \delta 158.2,146.1,142.6,138.1,134.7,131.1,130.0,127.3$, $121.9,121.4,121.3,120.7,120.6,120.2$, 120.1, 116.8, 113.3, 110.4, 61.1, 59.4, 55.2, 54.3, 46.1, 28.5, 24.6. HRMS (ESI-TOF) $\mathrm{m} / \mathrm{z}$ calcd for $\mathrm{C}_{27} \mathrm{H}_{28} \mathrm{~N}_{3} \mathrm{O}[\mathrm{M}+\mathrm{H}]^{+}:$410.2227; found: 410.2230 .

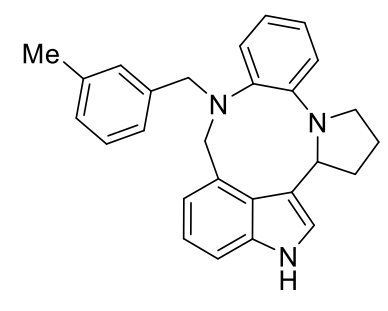

$3 \mathbf{g}$

7-(3-methylbenzyl)-6,7,13,14,15,15a-hexahydro-2H-benzo[2,3]pyrrolo [1',2':4,5][1, 4]diazonino[6,7,8-cd]indole (3g): According to general procedure (for $36 \mathrm{~h}$ ), $\mathbf{1 a}$ (58.4 mg, $0.2 \mathrm{mmol}), 2 \mathrm{~g}$ (48.1 mg, $0.4 \mathrm{mmol})$, TfOH (6.0 mg, $0.04 \mathrm{mmol})$, afforded $\mathbf{3 g}$ (58.3 $\mathrm{mg}, 74 \%$ ) as a light green solid after purification on silica gel (petroleum ether/EtOAc $=15: 1), \operatorname{mp} 94-98{ }^{\circ} \mathrm{C} .{ }^{1} \mathbf{H}$ NMR (500 MHz, CDCl 3$) \delta 7.87(\mathrm{~s}, 1 \mathrm{H}), 7.13(\mathrm{~d}, J=8.1$ $\mathrm{Hz}, 1 \mathrm{H}), 7.00-6.90(\mathrm{~m}, 4 \mathrm{H}), 6.90-6.84(\mathrm{~m}, 2 \mathrm{H}), 6.83-6.76(\mathrm{~m}, 3 \mathrm{H}), 6.73(\mathrm{dt}, J=$ 
9.0, 4.6 Hz, 1H), $6.70-6.63(\mathrm{~m}, 1 \mathrm{H}), 5.33(\mathrm{~d}, J=6.5 \mathrm{~Hz}, 1 \mathrm{H}), 5.20(\mathrm{~d}, J=14.0 \mathrm{~Hz}$, 1H), $4.16(\mathrm{~s}, 2 \mathrm{H}), 3.81(\mathrm{~d}, J=14.0 \mathrm{~Hz}, 1 \mathrm{H}), 3.64-3.48(\mathrm{~m}, 1 \mathrm{H}), 3.08-2.97(\mathrm{~m}, 1 \mathrm{H})$, $2.22-2.12(\mathrm{~m}, 1 \mathrm{H}), 2.12-1.97(\mathrm{~m}, 5 \mathrm{H}), 1.96-1.88(\mathrm{~m}, 1 \mathrm{H}) .{ }^{13} \mathbf{C}$ NMR (125 MHz, $\left.\mathbf{C D C l}_{3}\right) \delta 146.1,142.8,139.0,138.0,137.3,134.6,129.8,127.8,127.3,125.9,122.0$, $121.4,121.2,120.7,120.6,120.2$, 120.2, 116.8, 110.4, 100.9, 61.0, 59.4, 55.2, 46.1, 28.6, 24.6, 21.4. HRMS (ESI-TOF) $\mathrm{m} / \mathrm{z}$ calcd for $\mathrm{C}_{27} \mathrm{H}_{28} \mathrm{~N}_{3}[\mathrm{M}+\mathrm{H}]^{+}: 394.2278$; found: 394.2277.

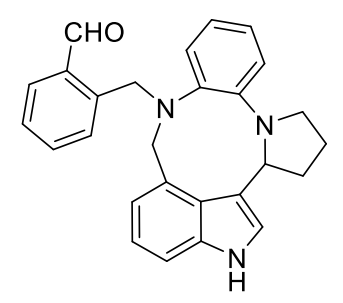

3h

2-((2,6,13,14,15,15a-hexahydro-7H-benzo[2,3]pyrrolo[1',2':4,5][1,4]diazonino[6,7, 8-cd]indol-7-yl)methyl)benzaldehyde (3h): According to general procedure (for 36 h), $1 \mathbf{a}(58.4 \mathrm{mg}, 0.2 \mathrm{mmol}), \mathbf{2 h}(53.7 \mathrm{mg}, 0.4 \mathrm{mmol}), \mathrm{TfOH}(6.0 \mathrm{mg}, 0.04 \mathrm{mmol})$, afforded $3 \mathrm{~h}(61.7 \mathrm{mg}, 76 \%)$ as a light yellow solid after purification on silica gel (petroleum ether/EtOAc = 15:1), mp 96 $-100{ }^{\circ} \mathrm{C} .{ }^{1} \mathbf{H}$ NMR (500 MHz, $\left.\mathbf{C D C l}_{3}\right) \delta 10.02$ (s, 1H), $7.95(\mathrm{~s}, 1 \mathrm{H}), 7.62(\mathrm{~d}, J=7.5 \mathrm{~Hz}, 1 \mathrm{H}), 7.36-7.25(\mathrm{~m}, 2 \mathrm{H}), 7.22-7.19(\mathrm{~m}, 1 \mathrm{H})$, $7.07(\mathrm{~d}, J=8.0 \mathrm{~Hz}, 1 \mathrm{H}), 6.99-6.93(\mathrm{~m}, 1 \mathrm{H}), 6.88(\mathrm{t}, J=7.6 \mathrm{~Hz}, 2 \mathrm{H}), 6.73(\mathrm{td}, J=14.0$, $12.9,6.9 \mathrm{~Hz}, 3 \mathrm{H}), 6.66-6.56(\mathrm{~m}, 1 \mathrm{H}), 5.52-5.33(\mathrm{~m}, 1 \mathrm{H}), 5.10(\mathrm{~d}, J=14.0 \mathrm{~Hz}, 1 \mathrm{H})$, $4.71(\mathrm{~d}, J=14.4 \mathrm{~Hz}, 1 \mathrm{H}), 4.51(\mathrm{~d}, J=14.4 \mathrm{~Hz}, 1 \mathrm{H}), 4.05$ (d, $J=14.0 \mathrm{~Hz}, 1 \mathrm{H}), 3.44$ (dd, $J=13.7,5.1 \mathrm{~Hz}, 1 \mathrm{H}), 2.99(\mathrm{dt}, J=11.5,8.4 \mathrm{~Hz}, 1 \mathrm{H}), 2.23-1.99(\mathrm{~m}, 3 \mathrm{H}), 1.98-1.88$ (m, 1H). ${ }^{13}$ C NMR (125 MHz, CDCl3) $\delta$ 153.0, 146.2, 142.4, 141.4, 138.0, 134.1, 127.2, 122.5, 121.4, 121.2, 120.9, 120.7, 120.4, 120.1, 116.8, 110.5, 110.1, 108.4, 60.7, 57.6, 47.5, 46.1, 28.4, 24.5. HRMS (ESI-TOF) $\mathrm{m} / \mathrm{z}$ calcd for $\mathrm{C}_{27} \mathrm{H}_{26} \mathrm{~N}_{3} \mathrm{O}[\mathrm{M}+\mathrm{H}]^{+}$: 408.2070; found: 408.2073 . 


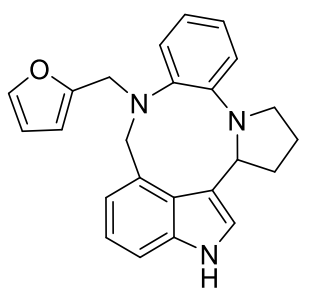

$3 \mathbf{i}$

7-(furan-2-ylmethyl)-6,7,13,14,15,15a-hexahydro-2H-benzo[2,3]pyrrolo[1',2':4,5]

$[1,4]$ diazonino[6,7,8-cd]indole (3i): According to general procedure (for $36 \mathrm{~h}$ ), 1a (58.4 mg, $0.2 \mathrm{mmol}), 2 \mathbf{i}(38.5 \mathrm{mg}, 0.4 \mathrm{mmol}), \mathrm{TfOH}$ (6.0 mg, $0.04 \mathrm{mmol})$, afforded $3 \mathbf{i}$ $(58.6 \mathrm{mg}, 79 \%)$ as a white oil after purification on silica gel (petroleum ether/EtOAc $=$ 15:1). ${ }^{1}$ H NMR (500 MHz, CDCl $) \delta 7.74(\mathrm{~s}, 1 \mathrm{H}), 7.15(\mathrm{~d}, J=1.8 \mathrm{~Hz}, 1 \mathrm{H}), 7.07$ (d, $J$ $=8.0 \mathrm{~Hz}, 1 \mathrm{H}), 7.05(\mathrm{~d}, J=7.7 \mathrm{~Hz}, 1 \mathrm{H}), 6.95(\mathrm{q}, J=7.8,6.5 \mathrm{~Hz}, 1 \mathrm{H}), 6.89-6.84(\mathrm{~m}$, 1H), $6.81(\mathrm{~d}, J=7.1 \mathrm{~Hz}, 1 \mathrm{H}), 6.77(\mathrm{~d}, J=4.3 \mathrm{~Hz}, 2 \mathrm{H}), 6.69(\mathrm{dq}, J=8.4,4.2 \mathrm{~Hz}, 1 \mathrm{H})$, $6.11(\mathrm{dd}, J=3.2,1.8 \mathrm{~Hz}, 1 \mathrm{H}), 5.98(\mathrm{~d}, J=3.1 \mathrm{~Hz}, 1 \mathrm{H}), 5.39$ (d, $J=6.2 \mathrm{~Hz}, 1 \mathrm{H}), 5.09$ $(\mathrm{d}, J=14.2 \mathrm{~Hz}, 1 \mathrm{H}), 4.25-4.09(\mathrm{~m}, 2 \mathrm{H}), 3.87(\mathrm{~d}, J=14.2 \mathrm{~Hz}, 1 \mathrm{H}), 3.52(\mathrm{td}, J=7.9$, $3.7 \mathrm{~Hz}, 1 \mathrm{H}), 2.98(\mathrm{dt}, J=12.1,8.2 \mathrm{~Hz}, 1 \mathrm{H}), 2.20-2.10(\mathrm{~m}, 1 \mathrm{H}), 2.10-1.98(\mathrm{~m}, 2 \mathrm{H})$, 1.95 - 1.87 (m, 1H). ${ }^{13} \mathbf{C}$ NMR (125 MHz, CDCl $) \delta$ 153.0, 146.2, 142.4, 141.4, 138.0, $134.1,127.2,122.5,121.4,121.2,120.9,120.7,120.4,120.1,116.8,110.5,110.1,108.4$, 60.7, 57.6, 47.5, 46.1, 28.4, 24.5. HRMS (ESI-TOF) $\mathrm{m} / \mathrm{z}$ calcd for $\mathrm{C}_{24} \mathrm{H}_{24} \mathrm{~N}_{3} \mathrm{O}[\mathrm{M}+\mathrm{H}]^{+}$: 370.1914; found: 370.1914 .

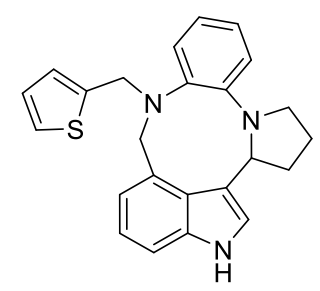

$3 \mathbf{j}$

\section{7-(thiophen-2-ylmethyl)-6,7,13,14,15,15a-hexahydro-2H-benzo[2,3]pyrrolo[1',2':}

4,5][1,4] diazonino[6,7,8-cd]indole (3j): According to general procedure (for $36 \mathrm{~h}$ ), $1 \mathbf{a}$ (58.4 mg, $0.2 \mathrm{mmol}$ ), $2 \mathbf{j}$ (44.9 mg, $0.4 \mathrm{mmol})$, TfOH (6.0 mg, $0.04 \mathrm{mmol}$ ), afforded 3j $(65.0 \mathrm{mg}, 84 \%)$ as a white solid after purification on silica gel (petroleum ether/EtOAc $=15: 1), \operatorname{mp} 105-107^{\circ} \mathrm{C}^{1}{ }^{1} \mathbf{H}$ NMR (500 MHz, $\left.\mathbf{C D C l}_{3}\right) \delta 7.78(\mathrm{~s}, 1 \mathrm{H}), 7.13-7.08(\mathrm{~m}$, $1 \mathrm{H}), 6.99-6.89(\mathrm{~m}, 4 \mathrm{H}), 6.83(\mathrm{~d}, J=7.1 \mathrm{~Hz}, 1 \mathrm{H}), 6.80-6.73(\mathrm{~m}, 3 \mathrm{H}), 6.71(\mathrm{dd}, J=$ 
5.0, 3.5 Hz, 1H), $6.66(\mathrm{td}, J=7.7,1.8 \mathrm{~Hz}, 1 \mathrm{H}), 5.48(\mathrm{~d}, J=4.8 \mathrm{~Hz}, 1 \mathrm{H}), 5.17$ (d, $J=$ $14.0 \mathrm{~Hz}, 1 \mathrm{H}), 4.47(\mathrm{~d}, J=15.1 \mathrm{~Hz}, 1 \mathrm{H}), 4.31(\mathrm{~d}, J=15.1 \mathrm{~Hz}, 1 \mathrm{H}), 3.83$ (d, $J=14.0 \mathrm{~Hz}$, $1 \mathrm{H}), 3.55(\mathrm{dq}, J=11.4,3.5 \mathrm{~Hz}, 1 \mathrm{H}), 3.00(\mathrm{dt}, J=12.1,8.3 \mathrm{~Hz}, 1 \mathrm{H}), 2.20-2.13(\mathrm{~m}$, 1H), $2.12-2.00(\mathrm{~m}, 2 \mathrm{H}), 1.95-1.86(\mathrm{~m}, 1 \mathrm{H}) .{ }^{\mathbf{1 3}} \mathbf{C}$ NMR (125 MHz, CDCl 3$) \delta 153.0$, 146.2, 142.4, 141.4, 138.0, 134.1, 127.2, 122.5, 121.4, 121.2, 120.9, 120.7, 120.4, 120.1, $116.8,110.5,110.1,108.4,60.7,57.6,47.5,46.1,28.4,24.5$. HRMS (ESI-TOF) m/z calcd for $\mathrm{C}_{24} \mathrm{H}_{24} \mathrm{~N}_{3} \mathrm{~S}[\mathrm{M}+\mathrm{H}]^{+}: 386.1685$; found: 386.1688 .

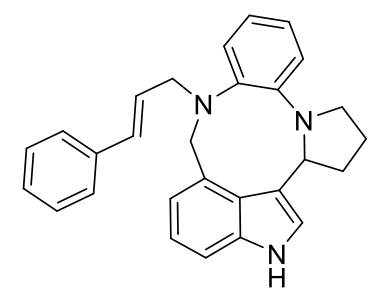

$3 \mathbf{k}$

\section{7-cinnamyl-6,7,13,14,15,15a-hexahydro-2H-benzo[2,3]pyrrolo[1',2':4,5][1,4]dia-}

zonino[6,7,8-cd]indole (3k): According to general procedure (for $36 \mathrm{~h}), \mathbf{1 a}(58.4 \mathrm{mg}$, $0.2 \mathrm{mmol}), 2 \mathbf{k}(52.9 \mathrm{mg}, 0.4 \mathrm{mmol}), \mathrm{TfOH}$ (6.0 mg, $0.04 \mathrm{mmol})$, afforded 3k (64.6 mg, $80 \%$ ) as a white solid after purification on silica gel (petroleum ether/EtOAc $=15: 1$ ), mp $72-76{ }^{\circ} \mathrm{C} .{ }^{1} \mathbf{H}$ NMR (500 MHz, $\left.\mathbf{C D C l}_{3}\right) \delta 7.94(\mathrm{~s}, 1 \mathrm{H}), 7.16-7.10(\mathrm{~m}, 5 \mathrm{H}), 7.08$ $-7.02(\mathrm{~m}, 1 \mathrm{H}), 7.00-6.93(\mathrm{~m}, 2 \mathrm{H}), 6.92-6.89(\mathrm{~m}, 1 \mathrm{H}), 6.86-6.80(\mathrm{~m}, 2 \mathrm{H}), 6.76$ $(\mathrm{dtd}, J=16.6,7.3,1.6 \mathrm{~Hz}, 2 \mathrm{H}), 6.42(\mathrm{~d}, J=15.8 \mathrm{~Hz}, 1 \mathrm{H}), 6.05(\mathrm{ddd}, J=15.9,7.2,5.5$ $\mathrm{Hz}, 1 \mathrm{H}), 5.25$ (d, $J=7.1 \mathrm{~Hz}, 1 \mathrm{H}), 5.16(\mathrm{~d}, J=14.1 \mathrm{~Hz}, 1 \mathrm{H}), 3.84$ (t, $J=6.5 \mathrm{~Hz}, 2 \mathrm{H})$, $3.76(\mathrm{~d}, J=14.2 \mathrm{~Hz}, 1 \mathrm{H}), 3.57(\mathrm{ddd}, J=11.3,7.7,2.8 \mathrm{~Hz}, 1 \mathrm{H}), 2.98(\mathrm{dt}, J=12.4,8.3$ $\mathrm{Hz}, 1 \mathrm{H}), 2.19-2.11(\mathrm{~m}, 1 \mathrm{H}), 2.12-2.01(\mathrm{~m}, 1 \mathrm{H}), 2.04-1.94(\mathrm{~m}, 1 \mathrm{H}), 1.96-1.85(\mathrm{~m}$, 1H). ${ }^{13} \mathbf{C}$ NMR (125 MHz, CDCl 3$) \delta$ 146.0, 143.4, 138.2, 137.3, 134.8, 131.9, 128.4, $128.2,127.3,127.2,126.3,121.9,121.5,121.3,120.8,120.6,120.4,120.1,116.9,110.5$, 61.5, 58.4, 52.9, 45.9, 28.0, 24.6. HRMS (ESI-TOF) $\mathrm{m} / \mathrm{z}$ calcd for $\mathrm{C}_{28} \mathrm{H}_{28} \mathrm{~N}_{3}[\mathrm{M}+\mathrm{H}]^{+}$: 406.2278; found: 406.2280 . 


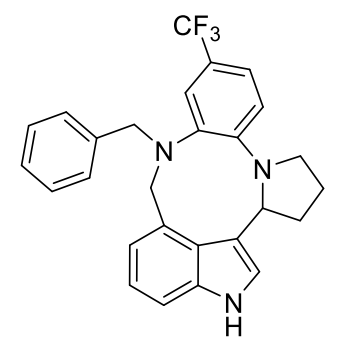

31

\section{7-benzyl-9-(trifluoromethyl)-6,7,13,14,15,15a-hexahydro-2H-benzo[2,3]pyrrolo}

$\left[1^{\prime}, 2^{\prime}: 4,5\right][1,4]$ diazonino[6,7,8-cd]indole (31): According to general procedure (for 36 h), 11 (71.88 mg, $0.2 \mathrm{mmol}), 2 \mathrm{a}(42.4 \mathrm{mg}, 0.4 \mathrm{mmol})$, TfOH (6.0 mg, $0.04 \mathrm{mmol})$, afforded 31 (66.0 mg, 74\%) as a light green solid after purification on silica gel $($ petroleum ether/EtOAc $=15: 1), \mathrm{mp} 136-140{ }^{\circ} \mathrm{C} .{ }^{1} \mathbf{H}$ NMR $\left(500 \mathbf{~ M H z}, \mathbf{C D C l}_{3}\right) \delta 8.08$ (s, 1H), 7.25 (t, $J=4.0 \mathrm{~Hz}, 2 \mathrm{H}), 7.20(\mathrm{~s}, 1 \mathrm{H}), 7.17-7.07(\mathrm{~m}, 5 \mathrm{H}), 7.05(\mathrm{t}, J=7.7 \mathrm{~Hz}$, 2H), $6.92(\mathrm{~d}, J=7.1 \mathrm{~Hz}, 1 \mathrm{H}), 6.82(\mathrm{~d}, J=8.4 \mathrm{~Hz}, 1 \mathrm{H}), 5.53(\mathrm{~d}, J=5.3 \mathrm{~Hz}, 1 \mathrm{H}), 5.12$ $(\mathrm{d}, J=14.1 \mathrm{~Hz}, 1 \mathrm{H}), 4.23(\mathrm{~m}, 2 \mathrm{H}), 3.96(\mathrm{~d}, J=14.1 \mathrm{~Hz}, 1 \mathrm{H}), 3.69-3.54(\mathrm{~m}, 1 \mathrm{H}), 3.24$ $-2.99(\mathrm{~m}, 1 \mathrm{H}), 2.37-2.20(\mathrm{~m}, 1 \mathrm{H}), 2.17-1.97(\mathrm{~m}, 3 \mathrm{H}) .{ }^{13} \mathbf{C}$ NMR (125 MHz, CDCl 3$)$ $\delta 149.1,141.8,138.1,138.0,133.7,129.2,128.1,127.0,126.9,124.9(\mathrm{q}, J=269.5 \mathrm{~Hz})$, 121.7, 121.6,121.4(q, $J=31.7 \mathrm{~Hz}), 120.8,119.7(\mathrm{q}, J=3.9 \mathrm{~Hz}), 119.6,117.9$ (q, $J=4.0$ $\mathrm{Hz}), 116.3,110.7,60.3,59.1,55.6,46.2,29.0,24.3 .{ }^{19} \mathbf{F}$ NMR $\left(470 \mathrm{MHz}, \mathrm{CDCl}_{3}\right) \delta-$ 61.27. HRMS (ESI-TOF) $\mathrm{m} / \mathrm{z}$ calcd for $\mathrm{C}_{27} \mathrm{H}_{25} \mathrm{~F}_{3} \mathrm{~N}_{3}[\mathrm{M}+\mathrm{H}]^{+}$: 448.1995; found: 448.1991.

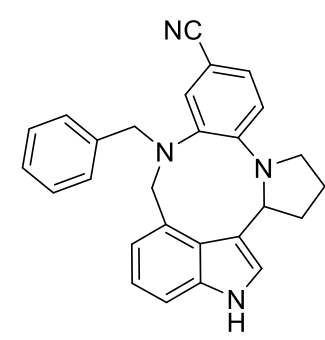

$3 \mathrm{~m}$

7-benzyl-6,7,13,14,15,15a-hexahydro-2H-benzo[2,3]pyrrolo[1',2':4,5][1,4]diazonino[6,7,8-cd]indole-9-carbonitrile (3m): According to general procedure (for $36 \mathrm{~h}$ ), $1 \mathbf{m}(63.3 \mathrm{mg}, 0.2 \mathrm{mmol}), \mathbf{2 a}(42.4 \mathrm{mg}, 0.4 \mathrm{mmol}), \mathrm{TfOH}(6.0 \mathrm{mg}, 0.04 \mathrm{mmol})$, afforded 3m (68.0 mg, 84\%) as a white solid after purification on silica gel (petroleum ether/EtOAc = 15:1), mp $198-202{ }^{\circ} \mathrm{C}^{1}{ }^{1} \mathbf{H}$ NMR (500 MHz, $\left.\mathbf{C D C l}_{3}\right) \delta 8.00(\mathrm{~s}, 1 \mathrm{H})$, 
$7.16-7.02(\mathrm{~m}, 8 \mathrm{H}), 7.02-6.99(\mathrm{~m}, 1 \mathrm{H}), 6.97(\mathrm{t}, J=7.5 \mathrm{~Hz}, 1 \mathrm{H}), 6.84(\mathrm{~d}, J=7.1 \mathrm{~Hz}$, $1 \mathrm{H}), 6.61(\mathrm{~d}, J=8.5 \mathrm{~Hz}, 1 \mathrm{H}), 5.84(\mathrm{dd}, J=8.4,3.2 \mathrm{~Hz}, 1 \mathrm{H}), 4.81(\mathrm{~d}, J=14.2 \mathrm{~Hz}, 1 \mathrm{H})$, $4.16(\mathrm{~d}, J=13.9 \mathrm{~Hz}, 1 \mathrm{H}), 4.10(\mathrm{~d}, J=14.2 \mathrm{~Hz}, 1 \mathrm{H}), 4.07$ (d, $J=14.0 \mathrm{~Hz}, 1 \mathrm{H}), 3.50-$ $3.36(\mathrm{~m}, 1 \mathrm{H}), 3.08(\mathrm{dt}, J=11.2,8.3 \mathrm{~Hz}, 1 \mathrm{H}), 2.29-2.21(\mathrm{~m}, 1 \mathrm{H}), 2.19-2.08(\mathrm{~m}, 1 \mathrm{H})$, 2.07 - 1.99 (m, 2H). ${ }^{13} \mathbf{C}$ NMR (125 MHz, CDCl $) \delta 150.5,140.8,137.7,137.5,132.8$, 129.2, 128.1, 128.1, 127.1, 126.7, 125.9, 121.8, 121.6, 120.7, 120.5, 118.9, 116.6, 110.6, 101.1, 59.0, 58.6, 57.0, 46.5, 29.8, 24.0. HRMS (ESI-TOF) $\mathrm{m} / \mathrm{z}$ calcd for $\mathrm{C}_{27} \mathrm{H}_{25} \mathrm{~N}_{4}$ $[\mathrm{M}+\mathrm{H}]^{+}:$405.2074; found: 405.2075.

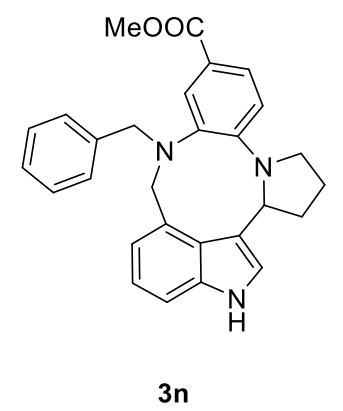

Methyl7-benzyl-6,7,13,14,15,15a-hexahydro-2H-benzo[2,3]pyrrolo[1',2':4,5][1,4] diazonino[6,7,8-cd]indole-9-carboxylate (3n): According to general procedure (for 36 h), 1n (69.9 mg, $0.2 \mathrm{mmol}), 2 \mathrm{a}(42.4 \mathrm{mg}, 0.4 \mathrm{mmol}), \mathrm{TfOH}(6.0 \mathrm{mg}, 0.04 \mathrm{mmol})$, afforded 3n (65.0 mg, 74\%) as a white solid after purification on silica gel (petroleum ether/EtOAc $=15: 1), \operatorname{mp} 108-112{ }^{\circ} \mathrm{C} .{ }^{1} \mathbf{H}$ NMR (500 MHz, $\left.\mathbf{C D C l}_{3}\right) \delta 8.02(\mathrm{~s}, 1 \mathrm{H})$, $7.65(\mathrm{~d}, J=1.9 \mathrm{~Hz}, 1 \mathrm{H}), 7.42(\mathrm{dd}, J=8.5,2.0 \mathrm{~Hz}, 1 \mathrm{H}), 7.13-7.00(\mathrm{~m}, 6 \mathrm{H}), 6.97$ (d, $J$ $=2.0 \mathrm{~Hz}, 1 \mathrm{H}), 6.93(\mathrm{t}, J=7.6 \mathrm{~Hz}, 1 \mathrm{H}), 6.83(\mathrm{~d}, J=7.1 \mathrm{~Hz}, 1 \mathrm{H}), 6.61(\mathrm{~d}, J=8.5 \mathrm{~Hz}$, 1H), $5.95-5.76(\mathrm{~m}, 1 \mathrm{H}), 4.85(\mathrm{~d}, J=14.1 \mathrm{~Hz}, 1 \mathrm{H}), 4.14(\mathrm{~s}, 2 \mathrm{H}), 4.03(\mathrm{~d}, J=14.1 \mathrm{~Hz}$, $1 \mathrm{H}), 3.73(\mathrm{~s}, 3 \mathrm{H}), 3.50-3.43(\mathrm{~m}, 1 \mathrm{H}), 3.05(\mathrm{dt}, J=11.3,8.1 \mathrm{~Hz}, 1 \mathrm{H}), 2.25-2.14(\mathrm{~m}$, 1H), 2.12 - 1.93 (m, 3H). ${ }^{13}$ C NMR (125 MHz, CDCl3) $\delta 167.5,150.8,140.6,138.2$, $137.8,133.6,129.5,128.0,126.8,125.6,123.5,121.6,121.5,120.6,120.5,119.3,115.7$, 110.5, 59.3, 59.1, 57.1, 51.6, 46.4, 29.6, 24.1. HRMS (ESI-TOF) m/z calcd for $\mathrm{C}_{28} \mathrm{H}_{28} \mathrm{~N}_{3} \mathrm{O}_{2}[\mathrm{M}+\mathrm{H}]^{+}:$438.2176; found: 438.2177 . 


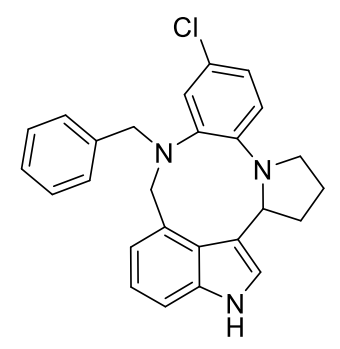

30

7-benzyl-9-chloro-6,7,13,14,15,15a-hexahydro-2H-benzo[2,3]pyrrolo[1',2':4,5][1, 4]diazonino[6,7,8-cd]indole (3o): According to general procedure (for $36 \mathrm{~h}), 10$ (65.2 mg, $0.2 \mathrm{mmol}$ ), 2a (42.4 mg, $0.4 \mathrm{mmol})$, TfOH (6.0 mg, $0.04 \mathrm{mmol})$, afforded $3 \mathbf{0}$ (56.0 $\mathrm{mg}, 68 \%$ ) as a white solid after purification on silica gel (petroleum ether/EtOAc $=$ 15:1), mp $86-90{ }^{\circ} \mathrm{C} .{ }^{1} \mathbf{H}$ NMR (500 MHz, $\left.\mathbf{C D C l}_{3}\right) \delta 7.85(\mathrm{~s}, 1 \mathrm{H}), 7.12(\mathrm{~d}, J=7.9 \mathrm{~Hz}$, 1H), $7.09-6.97$ (m, 5H), $7.08-6.98(\mathrm{~m}, 1 \mathrm{H}), 6.97-6.90(\mathrm{~m}, 1 \mathrm{H}), 6.85(\mathrm{~s}, 1 \mathrm{H}), 6.81$ $(\mathrm{d}, J=7.1 \mathrm{~Hz}, 1 \mathrm{H}), 6.68-6.62(\mathrm{~m}, 2 \mathrm{H}), 5.28-5.07(\mathrm{~m}, 2 \mathrm{H}), 4.23-4.08(\mathrm{~m}, 2 \mathrm{H}), 3.77$ $(\mathrm{d}, J=14.1 \mathrm{~Hz}, 1 \mathrm{H}), 3.56-3.32(\mathrm{~m}, 1 \mathrm{H}), 3.01-2.93(\mathrm{~m}, 1 \mathrm{H}), 2.18-2.08(\mathrm{~m}, 1 \mathrm{H})$, 2.05 - 1.82 (m, 3H). ${ }^{13} \mathbf{C}$ NMR (125 MHz, $\left.\mathbf{C D C l}_{3}\right) \delta 144.8,143.7,138.4,138.1,133.9$, $128.9,128.1,127.1,126.8,125.1,121.7,121.6,121.5,121.0,120.5,120.1,117.7,110.7$, 61.2, 59.2, 55.0, 46.3, 28.6, 24.5. HRMS (ESI-TOF) $\mathrm{m} / \mathrm{z}$ calcd for $\mathrm{C}_{26} \mathrm{H}_{25} \mathrm{ClN}_{3}[\mathrm{M}+$ $\mathrm{H}]^{+}:$414.1732; found: 414.1735 .

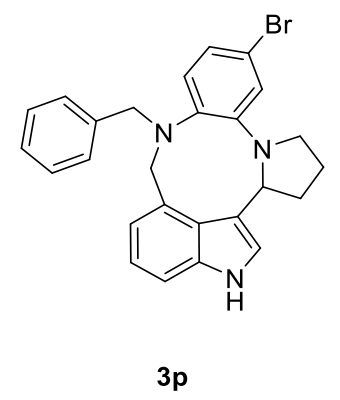

7-benzyl-10-bromo-6,7,13,14,15,15a-hexahydro-2H-benzo[2,3]pyrrolo[1',2' :4,5][1, 4]diazonino[6,7,8-cd]indole (3p): According to general procedure (for $36 \mathrm{~h}$ ), 1p (74.1 mg, $0.2 \mathrm{mmol}), 2 \mathrm{a}(42.4 \mathrm{mg}, 0.4 \mathrm{mmol}), \mathrm{TfOH}(6.0 \mathrm{mg}, 0.04 \mathrm{mmol})$, afforded 3p (69.0 $\mathrm{mg}, 75 \%$ ) as a white solid after purification on silica gel (petroleum ether/EtOAc $=$ 15:1), mp $158-162{ }^{\circ} \mathrm{C} .{ }^{1} \mathbf{H}$ NMR (500 MHz, $\left.\mathbf{C D C l}_{3}\right) \delta 7.82(\mathrm{~s}, 1 \mathrm{H}), 7.11-7.07(\mathrm{~m}$, 1H), $7.07-6.98(\mathrm{~m}, 5 \mathrm{H}), 6.96-6.90(\mathrm{~m}, 2 \mathrm{H}), 6.82-6.78(\mathrm{~m}, 2 \mathrm{H}), 6.70(\mathrm{~s}, 2 \mathrm{H}), 5.39$ $(\mathrm{d}, J=4.7 \mathrm{~Hz}, 1 \mathrm{H}), 5.04(\mathrm{~d}, J=14.1 \mathrm{~Hz}, 1 \mathrm{H}), 4.24-4.03(\mathrm{~m}, 2 \mathrm{H}), 3.84(\mathrm{~d}, J=14.1$ 
$\mathrm{Hz}, 1 \mathrm{H}), 3.44-3.37(\mathrm{~m}, 1 \mathrm{H}), 3.05-2.87(\mathrm{~m}, 1 \mathrm{H}), 2.21-2.08(\mathrm{~m}, 1 \mathrm{H}), 2.06-1.96(\mathrm{~m}$, 2H), 1.95 - 1.85 (m, 1H). ${ }^{13}$ C NMR (125 MHz, CDCl $) \delta 147.6,141.3,138.5,138.0$, 133.8, 129.0, 128.1, 127.1, 126.8, 122.7, 122.2, 121.6, 120.8, 120.0, 119.7, 115.4, 110.7, 60.4, 59.1, 55.7, 46.4, 29.0, 24.3. HRMS (ESI-TOF) $\mathrm{m} / \mathrm{z}$ calcd for $\mathrm{C}_{26} \mathrm{H}_{25} \mathrm{BrN}_{3}[\mathrm{M}+$ $\mathrm{H}]^{+}:$458.1226; found: 458.1228 .

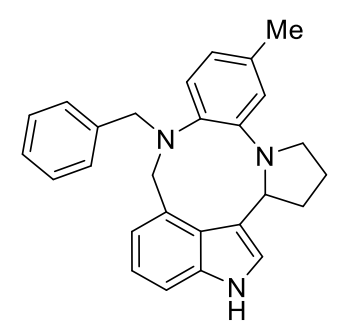

$3 q$

7-benzyl-10-methyl-6,7,13,14,15,15a-hexahydro-2H-benzo[2,3]pyrrolo[1',2':4,5]

[1,4]diazonino[6,7,8-cd]indole (3q): According to general procedure (for $36 \mathrm{~h}$ ), $\mathbf{1 q}$ (61.1 mg, $0.2 \mathrm{mmol}), \mathbf{2 a}(42.4 \mathrm{mg}, 0.4 \mathrm{mmol}), \mathrm{TfOH}$ (6.0 mg, $0.04 \mathrm{mmol})$, afforded 3q $(64.0 \mathrm{mg}, 81 \%)$ as a white solid after purification on silica gel (petroleum ether/EtOAc $=15: 1), \operatorname{mp} 85-90{ }^{\circ} \mathrm{C}^{1}{ }^{1} \mathbf{H}$ NMR (500 MHz, CDCl 3$) \delta 7.88(\mathrm{~s}, 1 \mathrm{H}), 7.13(\mathrm{~d}, J=8.1$ $\mathrm{Hz}, 1 \mathrm{H}), 7.10-7.01(\mathrm{~m}, 4 \mathrm{H}), 7.01-6.90$ (m, 3H), $6.81(\mathrm{dd}, J=7.6,2.5 \mathrm{~Hz}, 2 \mathrm{H}), 6.58$ (s, 1H), $6.46(\mathrm{~d}, J=7.7 \mathrm{~Hz}, 1 \mathrm{H}), 5.38(\mathrm{~d}, J=6.0 \mathrm{~Hz}, 1 \mathrm{H}), 5.16(\mathrm{~d}, J=14.0 \mathrm{~Hz}, 1 \mathrm{H})$, $4.17(\mathrm{~s}, 2 \mathrm{H}), 3.78(\mathrm{~d}, J=14.0 \mathrm{~Hz}, 1 \mathrm{H}), 3.59-3.52(\mathrm{~m}, 1 \mathrm{H}), 3.08-2.91(\mathrm{~m}, 1 \mathrm{H}), 2.20$ $-2.13(\mathrm{~m}, 1 \mathrm{H}), 2.11(\mathrm{~s}, 3 \mathrm{H}), 2.09-1.97(\mathrm{~m}, 2 \mathrm{H}), 1.96-1.88(\mathrm{~m}, 1 \mathrm{H}) .{ }^{13} \mathbf{C}$ NMR (125 MHz, $\left.\mathbf{C D C l}_{3}\right) \delta 145.8,140.0,139.2,138.0,134.7,131.4,128.9,127.9,127.3,126.5$, $121.4,121.3,120.7,120.6,120.5,120.1,117.8,110.4,60.9,59.6,55.5,46.1,28.6,24.5$, 21.2. HRMS (ESI-TOF) $\mathrm{m} / \mathrm{z}$ calcd for $\mathrm{C}_{27} \mathrm{H}_{28} \mathrm{~N}_{3}[\mathrm{M}+\mathrm{H}]^{+}:$394.2278; found: 394.2281.

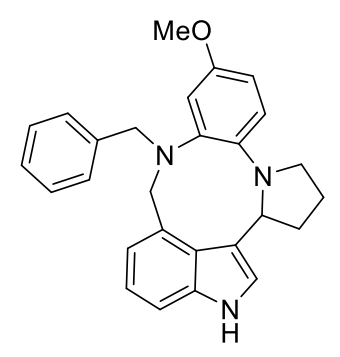

$3 r$

7-benzyl-9-methoxy-6,7,13,14,15,15a-hexahydro-2H-benzo[2,3]pyrrolo[1',2':4,5]

[1,4]diazonino[6,7,8-cd]indole (3r): According to general procedure (for $36 \mathrm{~h}$ ), $1 \mathbf{r}$ 
(64.3 mg, $0.2 \mathrm{mmol}), \mathbf{2 a}(42.4 \mathrm{mg}, 0.4 \mathrm{mmol}), \mathrm{TfOH}(6.0 \mathrm{mg}, 0.04 \mathrm{mmol})$, afforded $\mathbf{3 r}$ $(53.0 \mathrm{mg}, 65 \%)$ as a white solid after purification on silica gel (petroleum ether/EtOAc = 15:1), mp $95-100{ }^{\circ} \mathrm{C} .{ }^{1} \mathbf{H}$ NMR (500 MHz, $\left.\mathbf{C D C l}_{3}\right) \delta 8.03(\mathrm{~s}, 1 \mathrm{H}), 7.24(\mathrm{~s}, 1 \mathrm{H})$, $7.08(\mathrm{~s}, 1 \mathrm{H}), 7.06-7.01(\mathrm{~m}, 3 \mathrm{H}), 6.99(\mathrm{~d}, J=7.6 \mathrm{~Hz}, 1 \mathrm{H}), 6.90(\mathrm{~d}, J=7.1 \mathrm{~Hz}, 1 \mathrm{H})$, $6.86(\mathrm{~d}, J=7.7 \mathrm{~Hz}, 1 \mathrm{H}), 6.81(\mathrm{t}, J=7.0 \mathrm{~Hz}, 1 \mathrm{H}), 6.74(\mathrm{t}, J=7.5 \mathrm{~Hz}, 1 \mathrm{H}), 6.65(\mathrm{~d}, J=$ $8.5 \mathrm{~Hz}, 2 \mathrm{H}), 5.35(\mathrm{~d}, J=6.0 \mathrm{~Hz}, 1 \mathrm{H}), 5.29$ (d, $J=14.0 \mathrm{~Hz}, 1 \mathrm{H}), 4.22(\mathrm{~s}, 2 \mathrm{H}), 3.84$ (d, $J=14.0 \mathrm{~Hz}, 1 \mathrm{H}), 3.69$ (s, 3H), 3.64 (s, 1H), 3.08 (m, 1H), $2.29-2.18$ (m, 1H), 2.182.05 (m, 2H), 2.03 - 1.94 (m, 1H). ${ }^{13}$ C NMR (125 MHz, CDCl $) \delta 167.5,150.7,140.5$, 138.2, 137.8, 133.6, 129.5, 128.0, 126.8, 125.6, 123.5, 121.6, 121.5, 120.6, 120.5, 119.3, 115.7, 110.4, 59.3, 59.1, 57.0, 51.6, 46.4, 29.6, 24.1. HRMS (ESI-TOF) m/z calcd for $\mathrm{C}_{27} \mathrm{H}_{28} \mathrm{~N}_{3} \mathrm{O}[\mathrm{M}+\mathrm{H}]^{+}:$410.2227; found: 410.2228 .

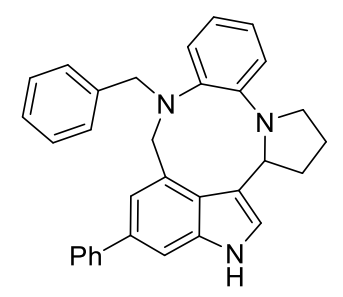

$3 \mathbf{s}$

\section{7-benzyl-4-phenyl-6,7,13,14,15,15a-hexahydro-2H-benzo[2,3]pyrrolo[1',2':4,5][1,} 4]diazonino[6,7,8-cd]indole (3s): According to general procedure (for $36 \mathrm{~h}$ ), $1 \mathrm{~s}$ (73.5 mg, $0.2 \mathrm{mmol}), \mathbf{2 a}(42.4 \mathrm{mg}, 0.4 \mathrm{mmol}), \mathrm{TfOH}(6.0 \mathrm{mg}, 0.04 \mathrm{mmol})$, afforded $3 \mathbf{s}$ (67.0 $\mathrm{mg}, 73 \%$ ) as a white solid after purification on silica gel (petroleum ether/EtOAc $=$ 15:1), mp $108-110{ }^{\circ} \mathrm{C}^{1}{ }^{1} \mathbf{H}$ NMR (500 MHz, $\left.\mathbf{C D C l}_{3}\right) \delta 7.91(\mathrm{~s}, 1 \mathrm{H}), 7.53$ (d, $J=7.3$ $\mathrm{Hz}, 2 \mathrm{H}), 7.36-7.31(\mathrm{~m}, 3 \mathrm{H}), 7.24-7.20$ (m, 1H), 7.07 (d, J=7.0 Hz, 3H), 7.03 (t, $J$ $=7.0 \mathrm{~Hz}, 3 \mathrm{H}), 7.00-6.95(\mathrm{~m}, 2 \mathrm{H}), 6.95-6.90(\mathrm{~m}, 1 \mathrm{H}), 6.81(\mathrm{~d}, J=7.7 \mathrm{~Hz}, 1 \mathrm{H}), 6.77$ $-6.70(\mathrm{~m}, 1 \mathrm{H}), 6.70-6.63(\mathrm{~m}, 1 \mathrm{H}), 5.38-5.22(\mathrm{~m}, 2 \mathrm{H}), 4.24(\mathrm{~s}, 2 \mathrm{H}), 3.83(\mathrm{~d}, J=14.1$ $\mathrm{Hz}, 1 \mathrm{H}), 3.67-3.49(\mathrm{~m}, 1 \mathrm{H}), 3.10-2.96(\mathrm{~m}, 1 \mathrm{H}), 2.21-2.12(\mathrm{~m}, 1 \mathrm{H}), 2.12-1.97(\mathrm{~m}$, 2H), 1.97 - 1.88(m, 1H). ${ }^{13}$ C NMR (125 MHz, CDCl $) \delta 146.0,142.8,142.0,139.0$, $138.7,134.9,134.8,128.9,128.7,127.9,127.3,126.7,126.6,126.6,122.0,122.0,120.5$, $120.3,120.1,116.9,108.8,61.2,59.8,55.2,46.2,28.5,24.6$. HRMS (ESI-TOF) m/z calcd for $\mathrm{C}_{32} \mathrm{H}_{30} \mathrm{~N}_{3}[\mathrm{M}+\mathrm{H}]^{+}: 456.2434$; found: 456.2435 . 


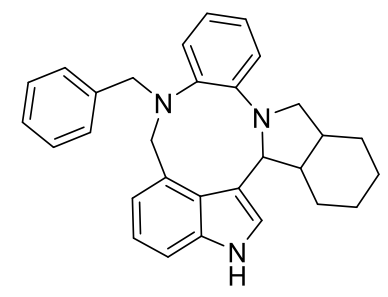

$3 \mathbf{t}$

5-benzyl-4,11,11a,12,13,14,15,15a,15b,17-decahydro-5H-benzo[2,3]isoindolo[2',

$1 ': 4,5][1,4]$ diazonino[6,7,8-cd]indole (3t): According to general procedure (for $36 \mathrm{~h}$ ), $1 t$ (69.1 mg, $0.2 \mathrm{mmol}), 2 \mathbf{a}(42.4 \mathrm{mg}, 0.4 \mathrm{mmol})$, TfOH (6.0 mg, $0.04 \mathrm{mmol})$, afforded 3t $(69.0 \mathrm{mg}, 81 \%)$ as a white solid after purification on silica gel (petroleum ether/EtOAc $=15: 1), \operatorname{mp} 122-126{ }^{\circ} \mathrm{C} .{ }^{1} \mathbf{H}$ NMR (500 MHz, DMSO) $\delta 10.96(\mathrm{~s}, 1 \mathrm{H})$, $7.34(\mathrm{~s}, 1 \mathrm{H}), 7.27-7.21(\mathrm{~m}, 2 \mathrm{H}), 7.19(\mathrm{~d}, J=6.9 \mathrm{~Hz}, 1 \mathrm{H}), 7.16(\mathrm{~d}, J=7.3 \mathrm{~Hz}, 5 \mathrm{H})$, $6.89(\mathrm{t}, J=7.6 \mathrm{~Hz}, 1 \mathrm{H}), 6.79(\mathrm{~d}, J=7.0 \mathrm{~Hz}, 1 \mathrm{H}), 6.76-6.67(\mathrm{~m}, 3 \mathrm{H}), 6.45(\mathrm{t}, J=7.1$ $\mathrm{Hz}, 1 \mathrm{H}), 5.43(\mathrm{~d}, J=5.6 \mathrm{~Hz}, 1 \mathrm{H}), 4.70(\mathrm{~d}, J=14.0 \mathrm{~Hz}, 1 \mathrm{H}), 4.29$ (d, $J=14.1 \mathrm{~Hz}, 1 \mathrm{H})$, $4.15(\mathrm{q}, J=14.6 \mathrm{~Hz}, 2 \mathrm{H}), 3.35(\mathrm{~s}, 1 \mathrm{H}), 3.21(\mathrm{dd}, J=9.6,4.3 \mathrm{~Hz}, 1 \mathrm{H}), 2.90-2.73(\mathrm{~m}$, 1H), $2.50-2.40$ (m, 1H), $1.76-1.57$ (m, 4H), 1.52 - 1.32 (m, 4H). ${ }^{13}$ C NMR (125 MHz, DMSO) $\delta 146.4,139.6,138.9,138.1,133.0,129.4,128.4,127.4,127.2,123.9$, 123.6, 120.6, 119.2, 118.4, 116.9, 116.1, 111.0, 62.1, 58.1, 57.2, 52.1, 42.9, 36.6, 26.8, 26.6, 23.7, 23.0. HRMS (ESI-TOF) $\mathrm{m} / \mathrm{z}$ calcd for $\mathrm{C}_{30} \mathrm{H}_{32} \mathrm{~N}_{3}[\mathrm{M}+\mathrm{H}]^{+}:$434.2591; found: 434.2594. 
6. NMR spectra for all compounds.

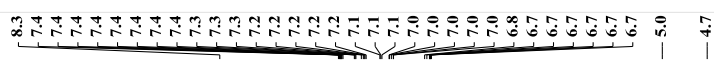
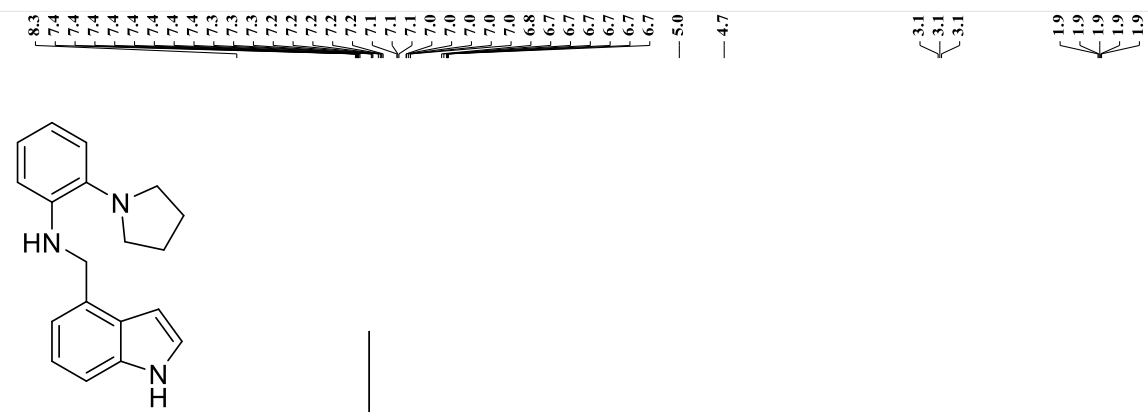

1a

${ }^{1} \mathrm{H}$ NMR

$\mathrm{CDCl}_{3}, 500 \mathrm{MHz}$
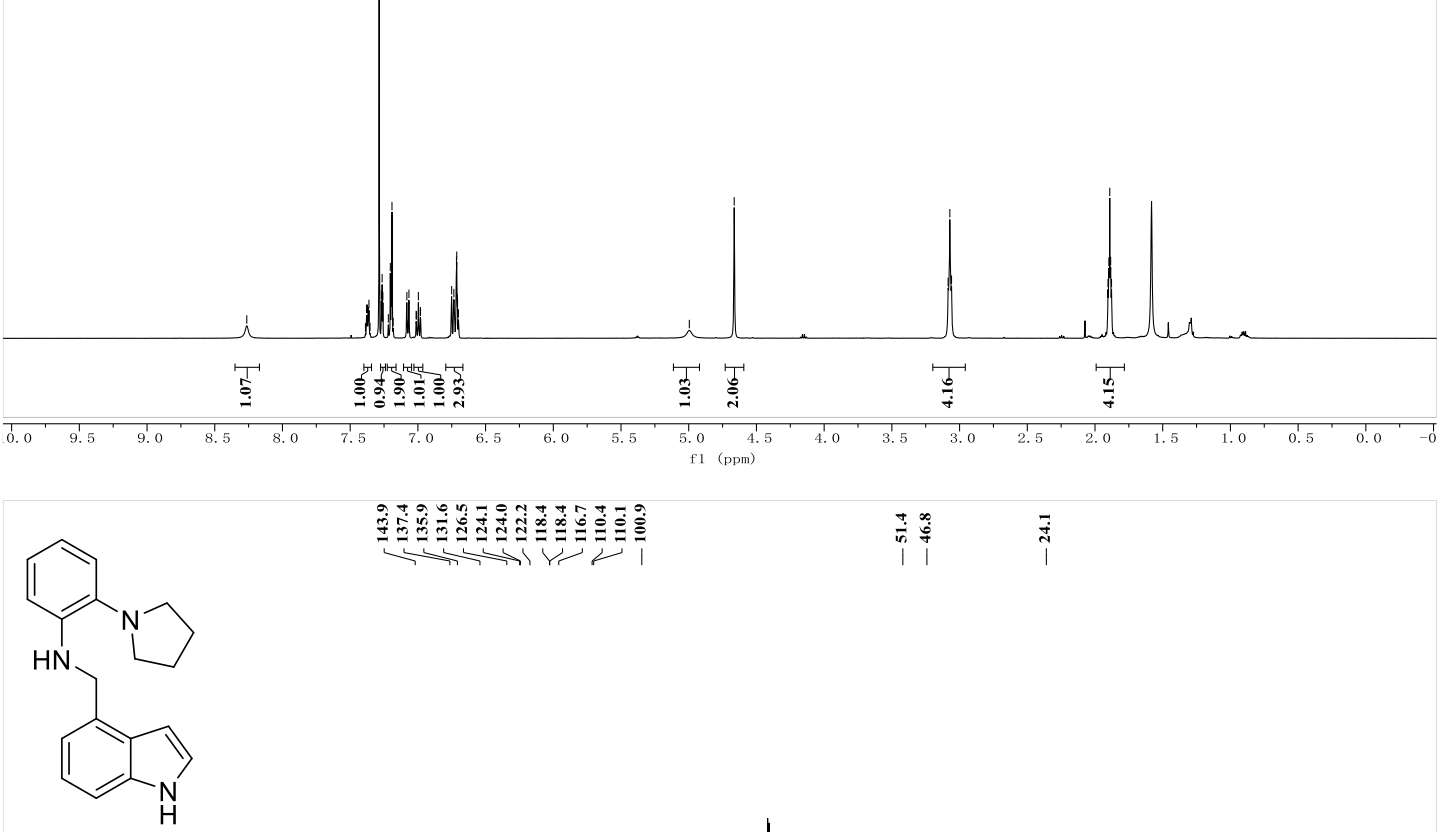

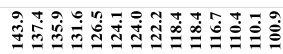

$\underset{\substack{10 \\ 1}}{1}$

$1 \mathrm{a}$

${ }^{13} \mathrm{C}$ NMR

$\mathrm{CDCl}_{3}, 125 \mathrm{MHz}$ 


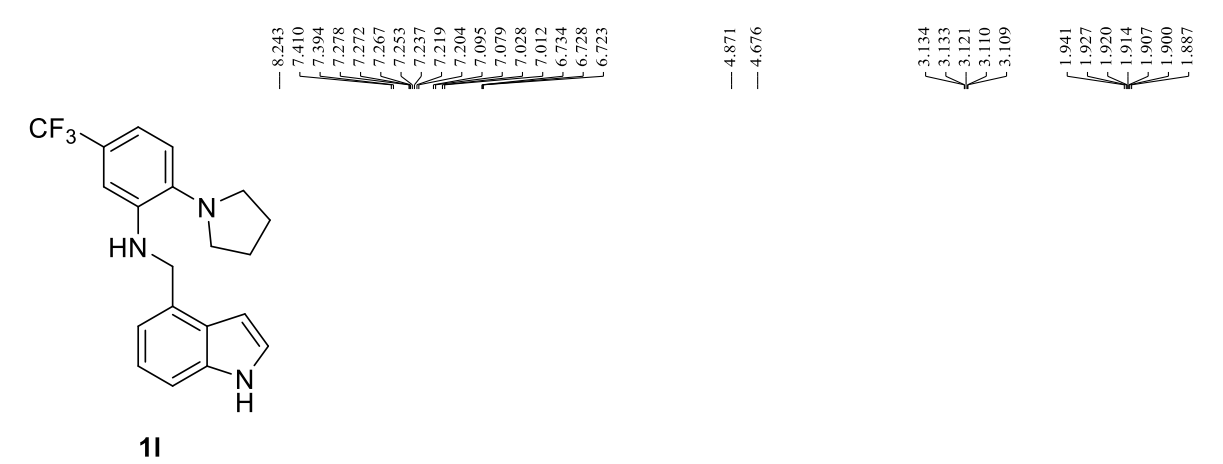

${ }^{1} \mathrm{H}$ NMR

$\mathrm{CDCl}_{3}, 500 \mathrm{MHz}$
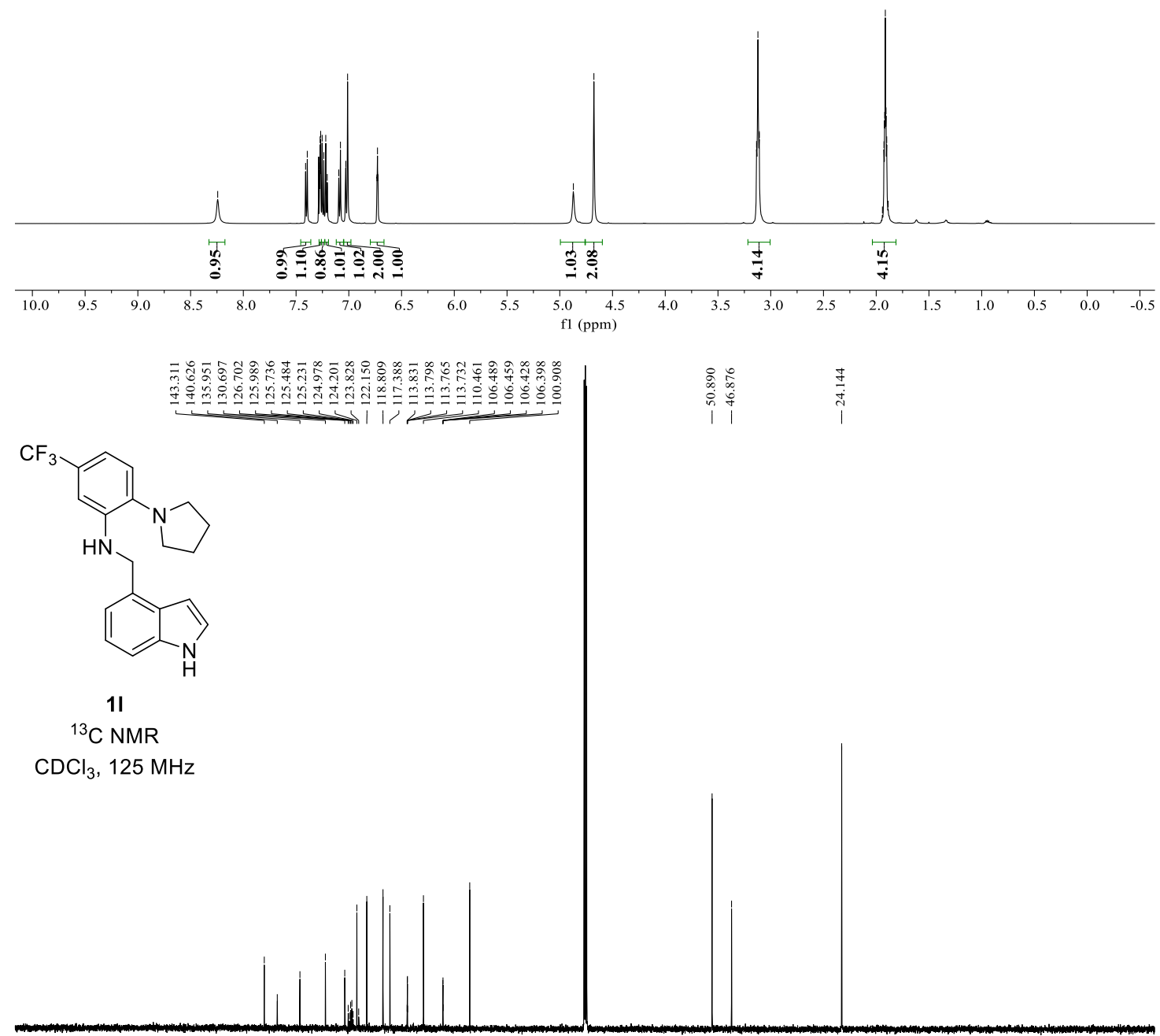

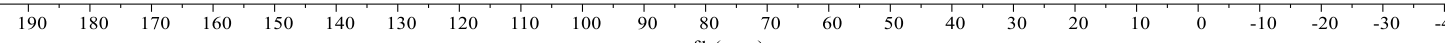




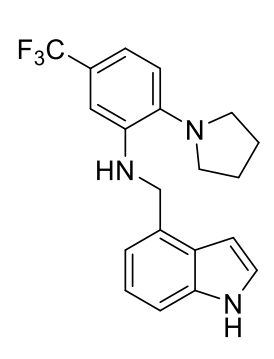

$\stackrel{\infty}{\dot{\varphi}}$

11

${ }^{19} \mathrm{~F}$ NMR

$\mathrm{CDCl}_{3}, 470 \mathrm{MHz}$

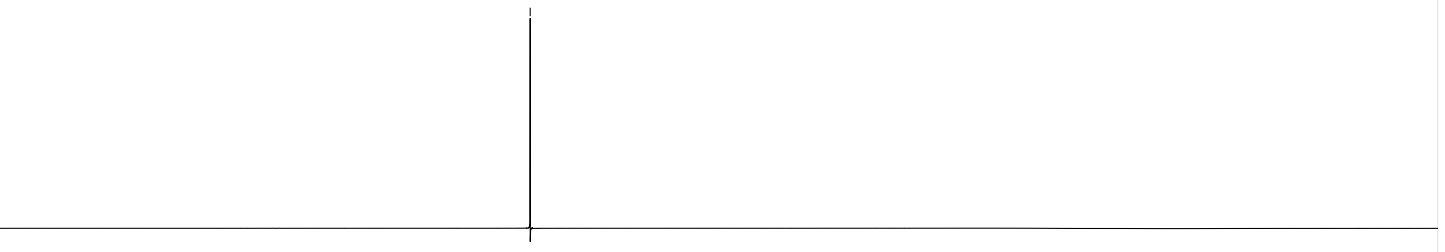

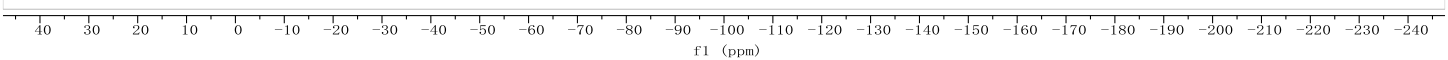


NC<smiles>Cc1cccc(NCc2cccc3[nH]ccc23)c1N1CCCC1</smiles>

$1 \mathrm{~m}$

${ }^{1} \mathrm{H}$ NMR $\mathrm{CDCl}_{3}, 500 \mathrm{MHz}$

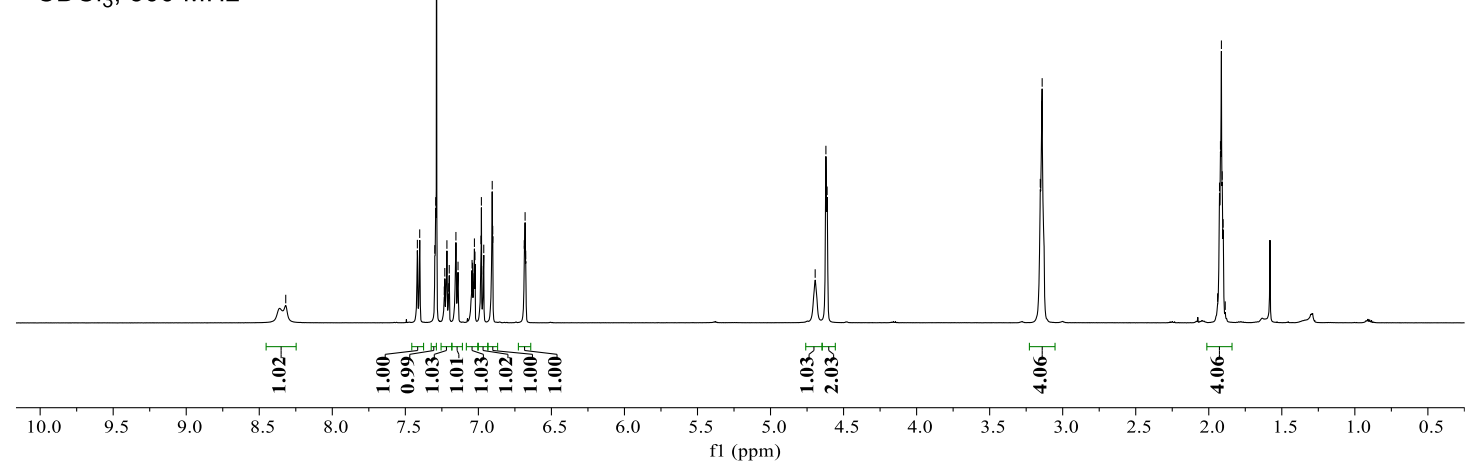

$\mathrm{NC}$<smiles>Cc1ccc(NCc2cccc3[nH]ccc23)c(N2CCCC2)c1</smiles>

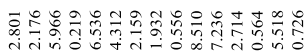

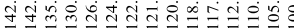

$4<0$

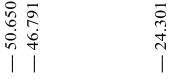

$1 \mathrm{~m}$

${ }^{13} \mathrm{C}$ NMR

$\mathrm{CDCl}_{3}, 125 \mathrm{MHz}$ 


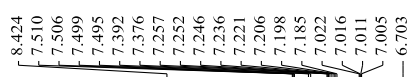

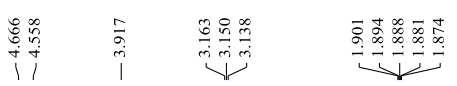<smiles>CC(=O)c1ccc(N2CCCC2)c(NCc2cccc3[nH]ccc23)c1</smiles>

$1 \mathrm{n}$

${ }^{1} \mathrm{H}$ NMR

$\mathrm{CDCl}_{3}, 500 \mathrm{MHz}$<smiles>c1ccc(N2CCCC2)c(NCc2cccc3[nH]ccc23)c1</smiles>

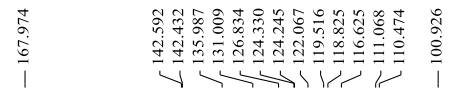

${ }^{13} \mathrm{C}$ NMR

$\mathrm{CDCl}_{3}, 125 \mathrm{MHz}$

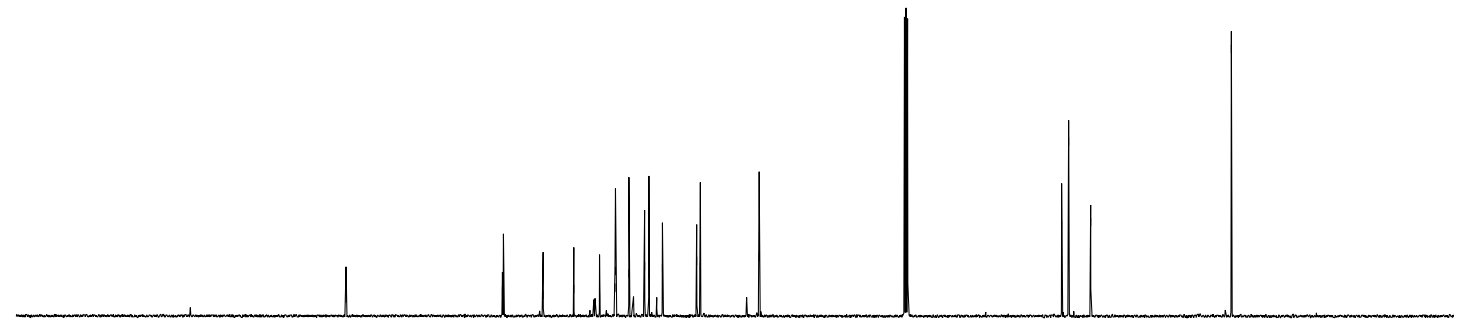

\begin{tabular}{llllllllllll}
220 & 210 & 200 & 190 & 180 & 170 & 160 & 150 & 140 & 130 & 120 & $\begin{array}{c}110 \\
\mathrm{fl}(\mathrm{ppm})\end{array}$ \\
\hline
\end{tabular} 


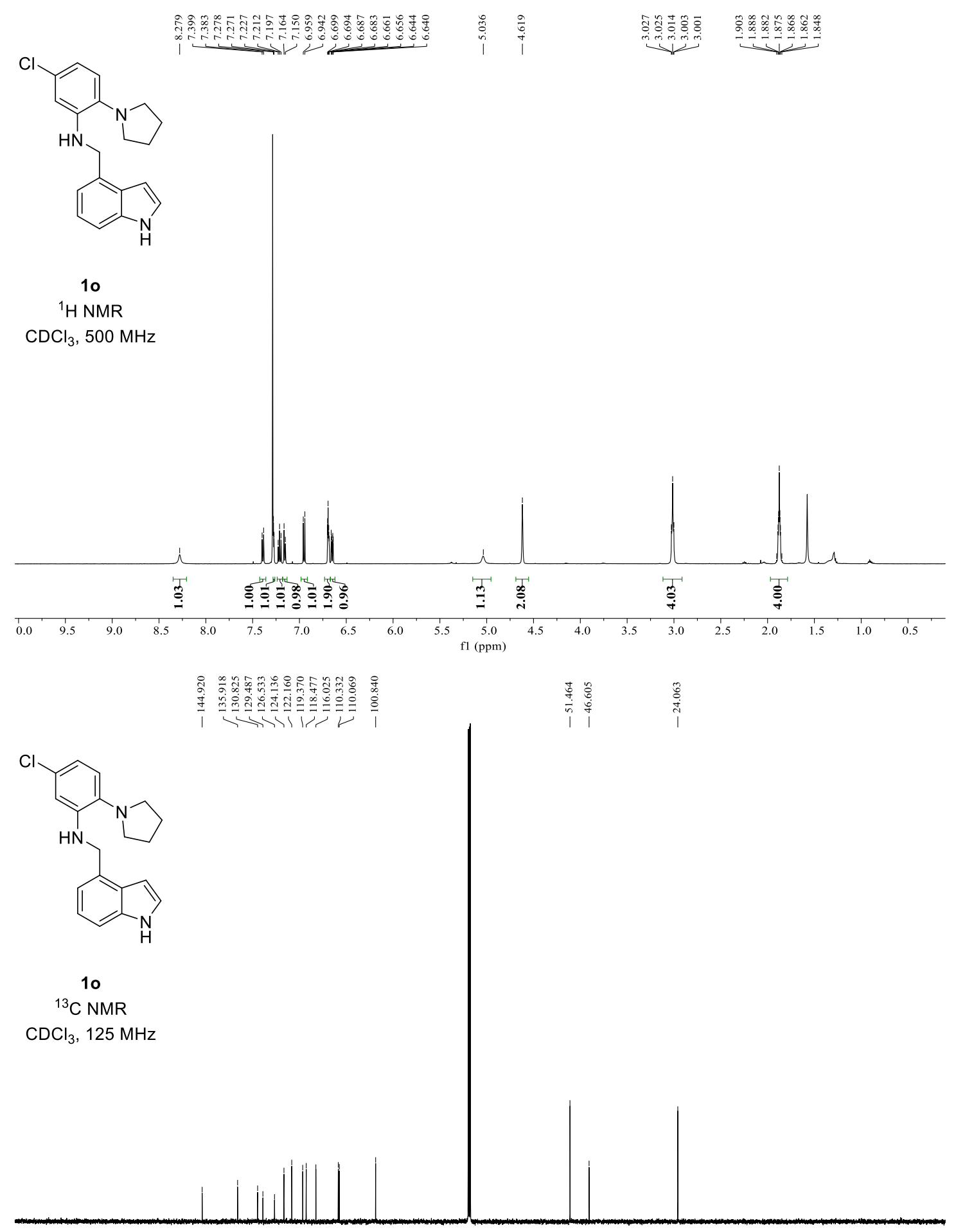

$\begin{array}{rrrrrrrrrrrrrrrrrrrrrrrr}1 & 1 \\ 190 & 180 & 170 & 160 & 150 & 140 & 130 & 120 & 110 & 100 & 90 & 80 & 70 & 60 & 50 & 40 & 30 & 20 & 10 & 0 & -10 & -20 & -30 & -40\end{array}$ 
<smiles>Brc1ccc(NCc2cccc3[nH]ccc23)c(N2CCCC2)c1</smiles>

$1 \mathrm{p}$

${ }^{1} \mathrm{H}$ NMR $\mathrm{CDCl}_{3}, 500 \mathrm{MHz}$

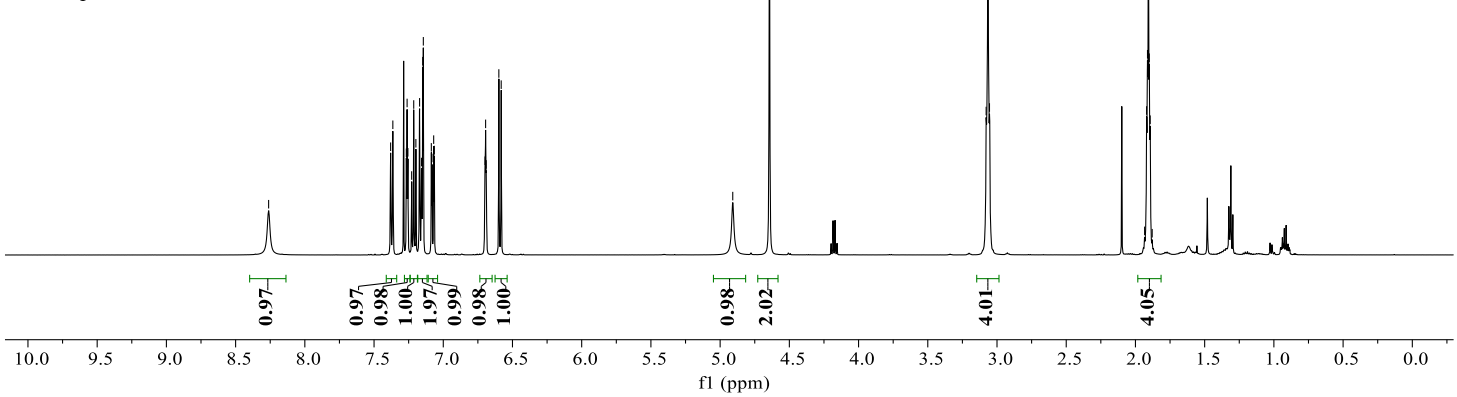

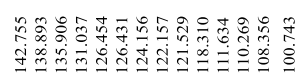

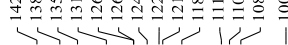<smiles>Brc1ccc(NCc2cccc3[nH]ccc23)c(N2CCCC2)c1</smiles>

$1 \mathrm{p}$

${ }^{13} \mathrm{C}$ NMR

$\mathrm{CDCl}_{3}, 125 \mathrm{MHz}$

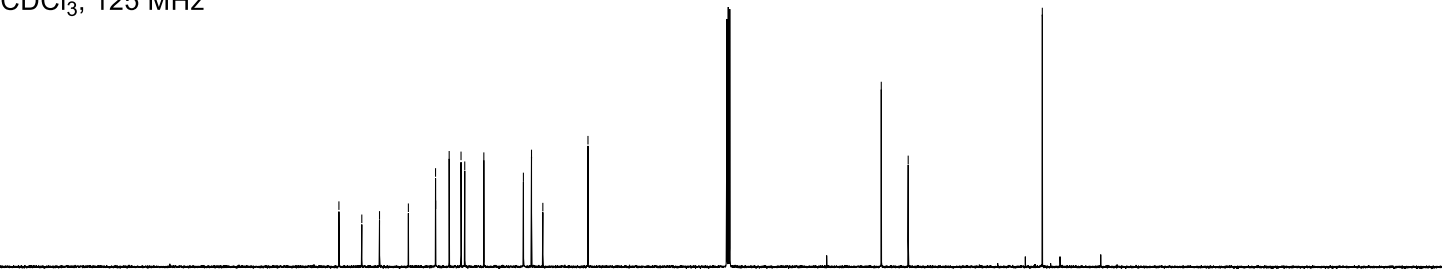

\begin{tabular}{lllllllllllllllllllllllllll}
\hline 00 & 190 & 180 & 170 & 160 & 150 & 140 & 130 & 120 & 110 & 100 & 90 & 80 & 70 & 60 & 50 & 40 & 30 & 20 & 10 & 0 & -10 & -20 & -30 & -40
\end{tabular} 
<smiles>Cc1ccc(NCc2cccc3[nH]ccc23)c(N2CCCC2)c1</smiles>

|

${ }^{1} \mathrm{H}$ NMR

$\mathrm{CDCl}_{3}, 500 \mathrm{MHz}$

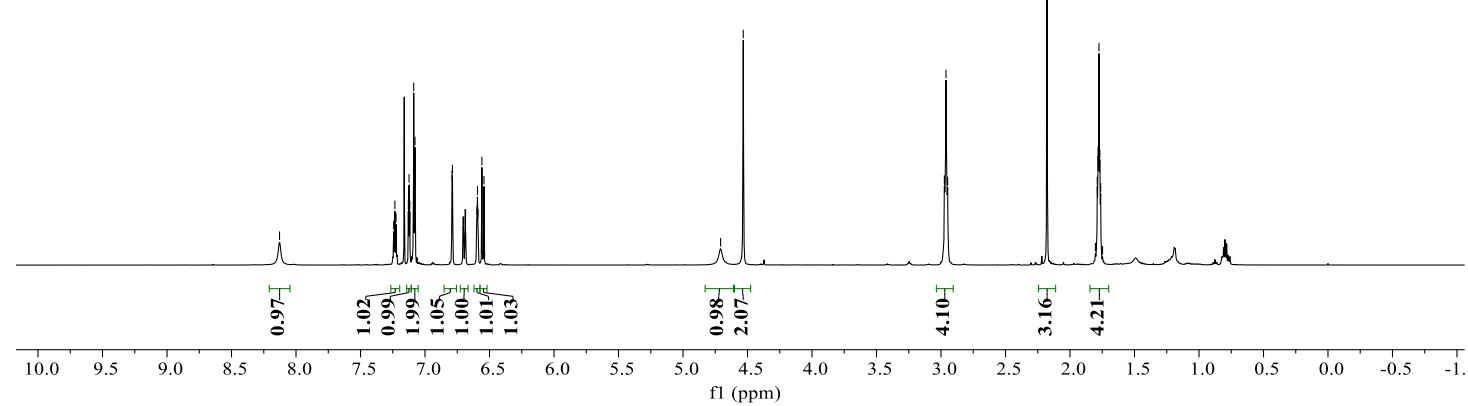<smiles>O=[N+]([O-])c1ccc(NCc2cccc3[nH]ccc23)c(N2CCCC2)c1</smiles>

$1 q$

${ }^{13} \mathrm{C}$ NMR

$\mathrm{CDCl}_{3}, 125 \mathrm{MHz}$

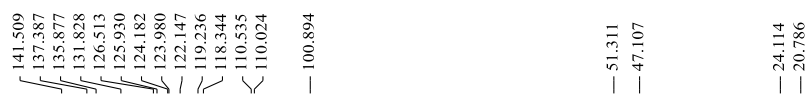

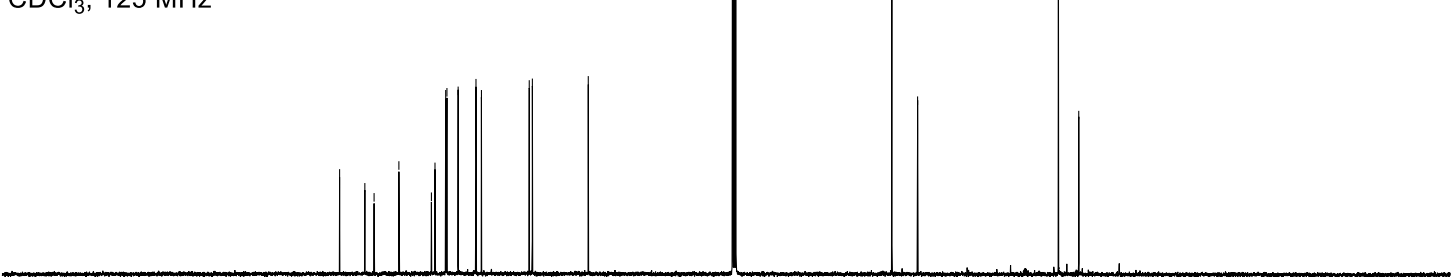

$\begin{array}{lllllllllllllllllllllllll}190 & 180 & 170 & 160 & 150 & 140 & 130 & 120 & 110 & 100 & 90 & 80 & 70 & 60 & 50 & 40 & 30 & 20 & 10 & 0 & -10 & -20 & -30\end{array}$ 


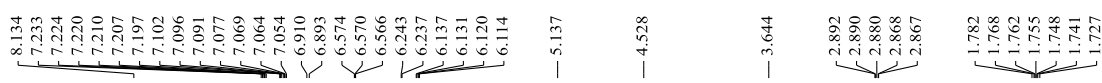<smiles>COc1ccc(N2CCCC2)c(NCc2cccc3[nH]ccc23)c1</smiles>

$1 \mathrm{r}$

${ }^{1} \mathrm{H}$ NMR

$\mathrm{CDCl}_{3}, 500 \mathrm{MHz}$

$\mathrm{MeO}$<smiles>c1ccc(N2CCCC2)c(NCNc2cccc3[nH]ccc23)c1</smiles>

向它年

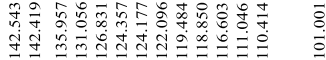

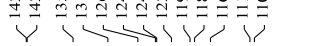

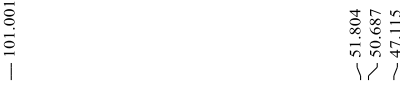

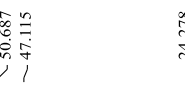

$1 \mathrm{r}$

${ }^{13} \mathrm{C}$ NMR

$\mathrm{CDCl}_{3}, 125 \mathrm{MHz}$

$\begin{array}{lllllllllll}190 & 180 & 170 & 160 & 150 & 140 & 130 & 120 & 110 & 100 & 90 \\ \mathrm{fl} 1(\mathrm{ppm}) & & 1 & 1\end{array}$ 
<smiles>c1ccc(-c2cc(CNc3ccccc3N3CCCC3)c3cc[nH]c3c2)cc1</smiles>

$1 \mathrm{~s}$

${ }^{1} \mathrm{H}$ NMR $\mathrm{CDCl}_{3}, 500 \mathrm{MHz}$

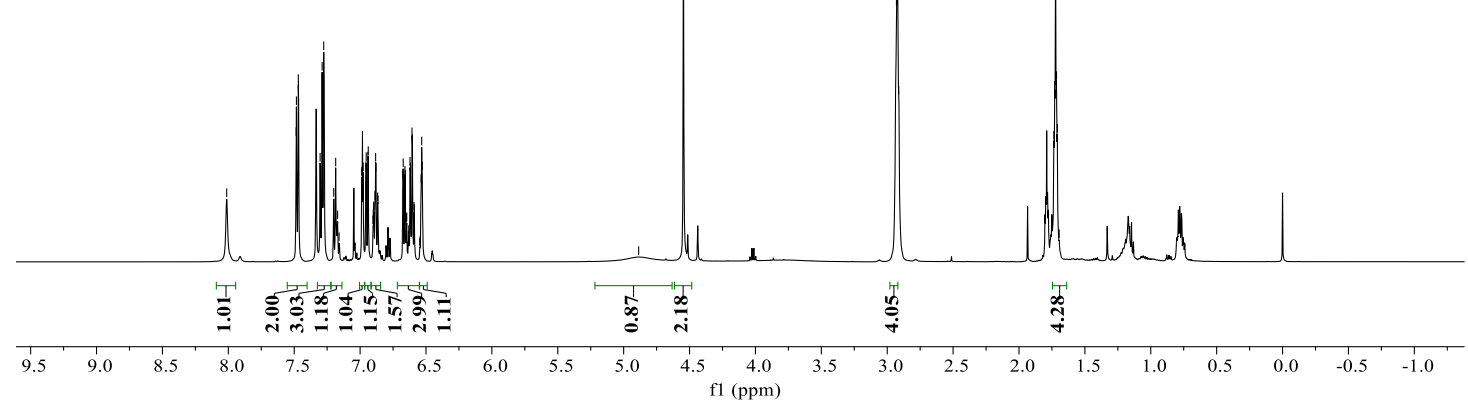

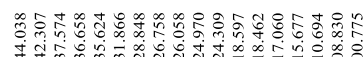

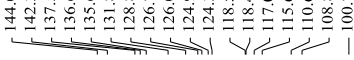<smiles>c1ccc(-c2cc(CNc3ccccc3N3CCCC3)c3cc[nH]c3c2)cc1</smiles>

$1 \mathrm{~s}$

${ }^{13} \mathrm{C}$ NMR $\mathrm{CDCl}_{3}, 125 \mathrm{MHz}$

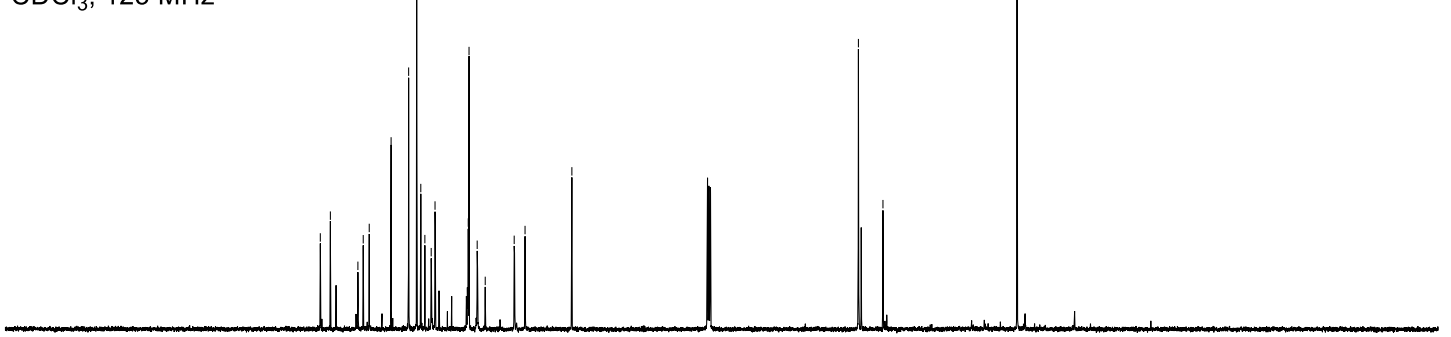

$\begin{array}{lllllllllllllllllllllllllll}190 & 180 & 170 & 160 & 150 & 140 & 130 & 120 & 110 & 100 & 90 & 80 & 70 & 60 & 50 & 40 & 30 & 20 & 10 & 0 & -10 & -20 & -30 & -40\end{array}$ 
<smiles>c1ccc(CNc2ccccc2NCc2cccc3[nH]ccc23)cc1</smiles>

$$
\begin{gathered}
\mathbf{1 t} \\
{ }^{1} \mathrm{H} \text { NMR } \\
\mathrm{CDCl}_{3}, 500 \mathrm{MHz}
\end{gathered}
$$

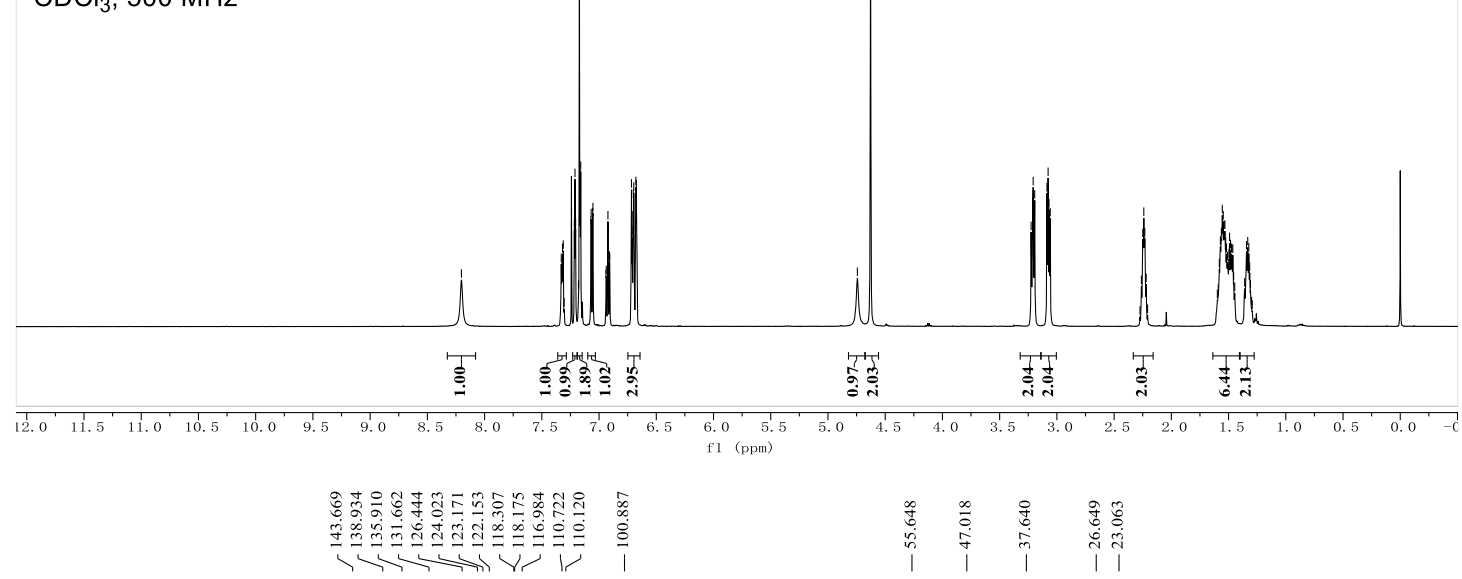<smiles>c1ccc(N2CC3CCCCC3C2)c(NCc2cccc3[nH]ccc23)c1</smiles>

$1 \mathrm{t}$

${ }^{13} \mathrm{C}$ NMR $\mathrm{CDCl}_{3}, 125 \mathrm{MHz}$

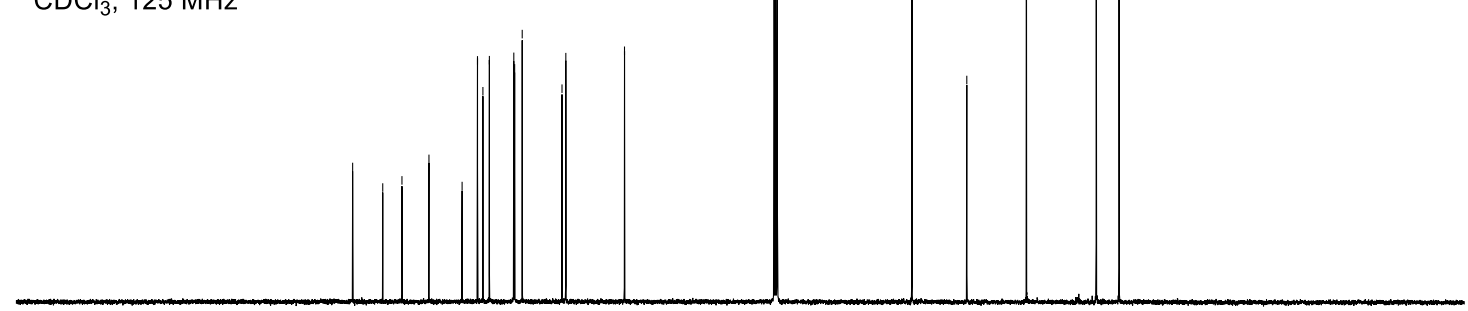

$\begin{array}{llllllllllllllllllllllllllll}190 & 180 & 170 & 160 & 150 & 140 & 130 & 120 & 110 & 100 & 90 & 80 & 70 & 60 & 50 & 40 & 30 & 20 & 10 & 0 & -10 & -20 & -3\end{array}$ 


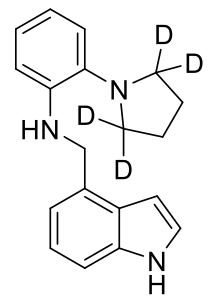

[D]-1a

${ }^{1} \mathrm{H}$ NMR

$\mathrm{CDCl}_{3}, 500 \mathrm{MHz}$
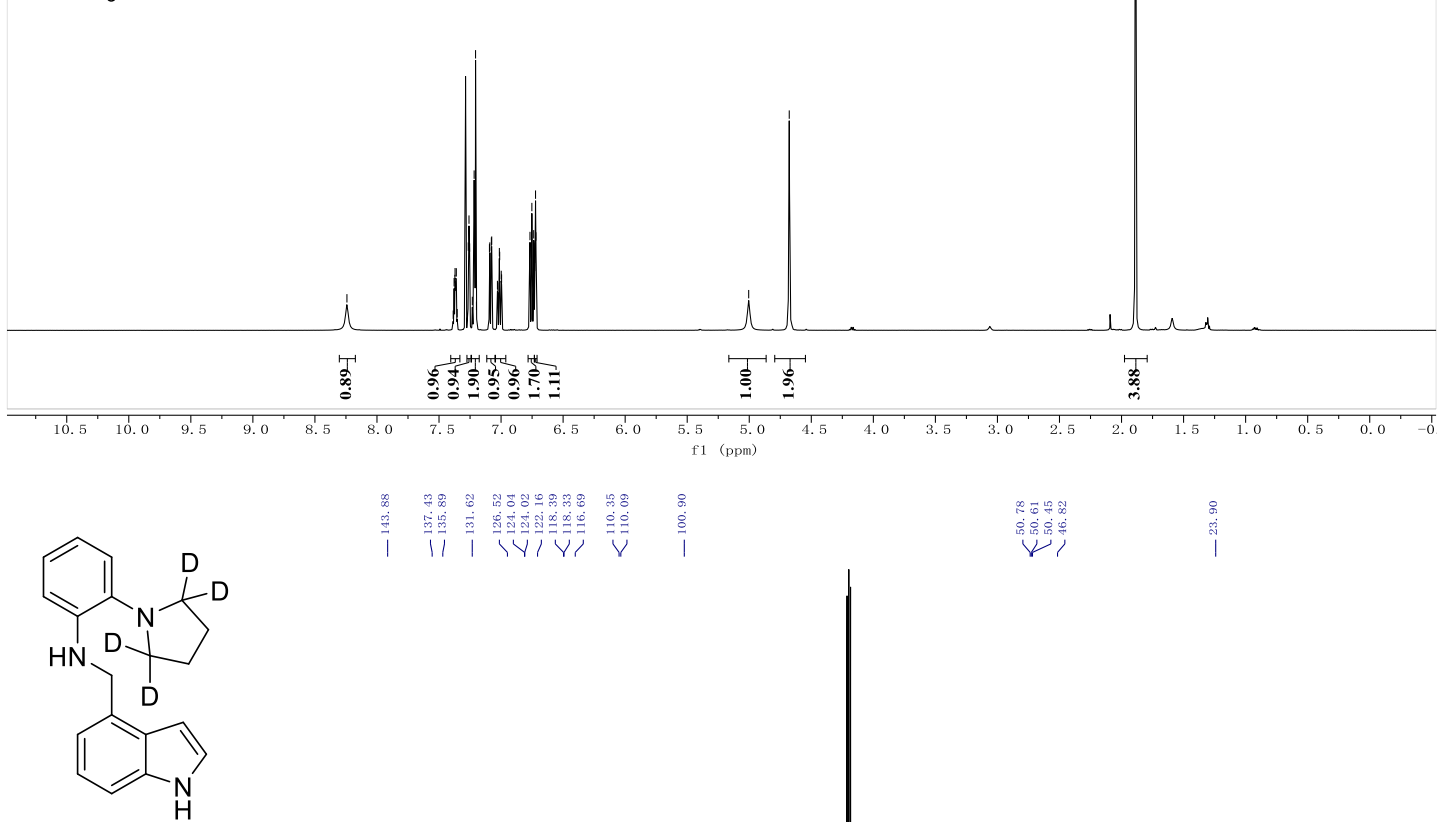

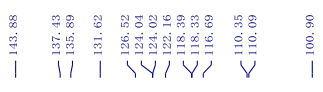

${ }^{13} \mathrm{C}$ NMR

$\mathrm{CDCl}_{3}, 125 \mathrm{MHz}$
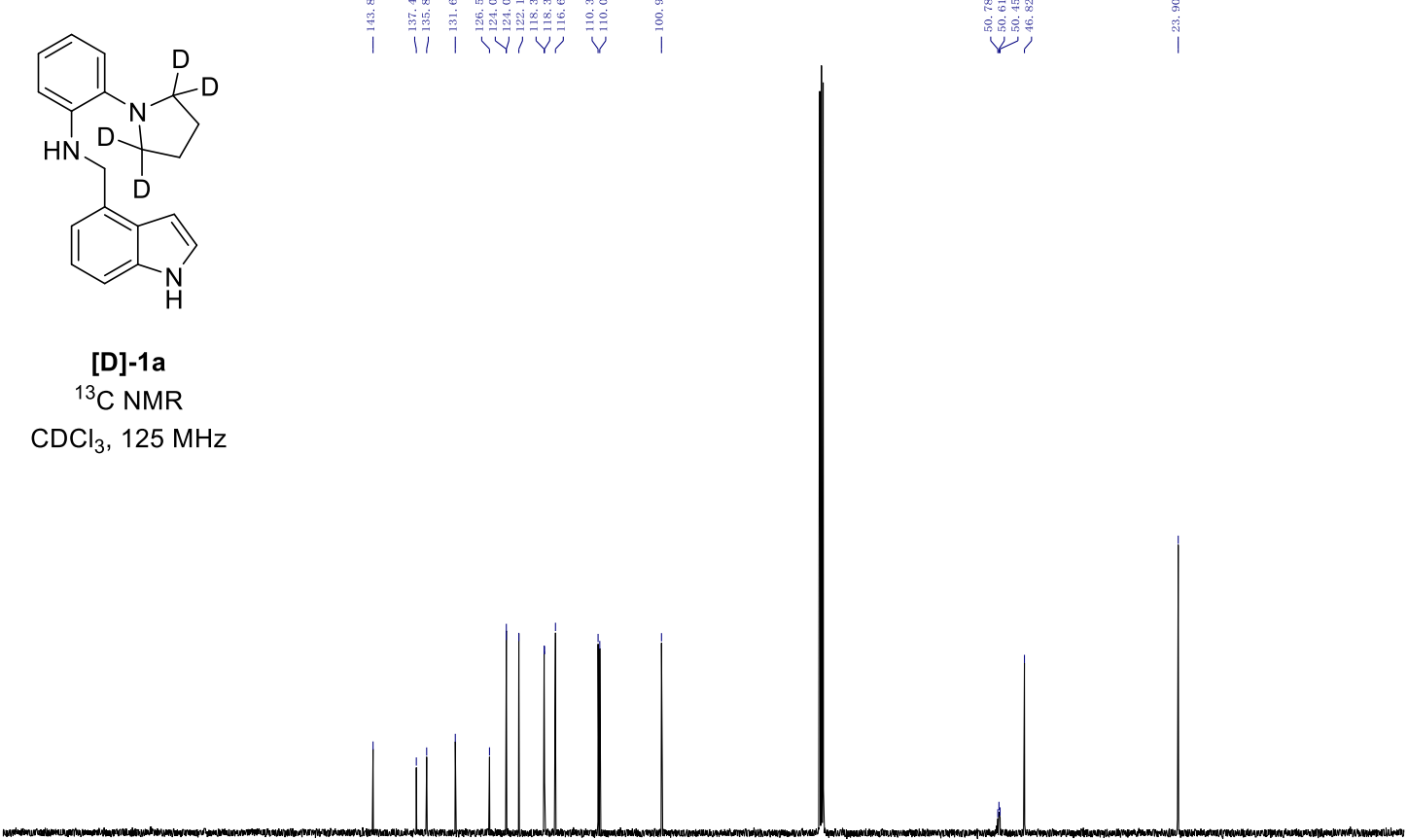

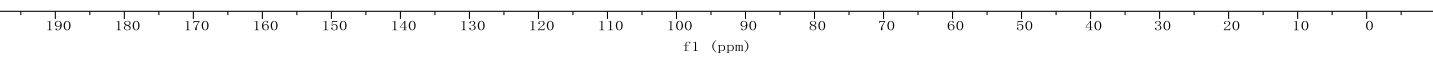


<smiles>c1ccc2c(c1)NC1(O2)C2CCCN21</smiles>

[D]-1b

${ }^{1} \mathrm{H}$ NMR

$\mathrm{CDCl}_{3}, 500 \mathrm{MHz}$

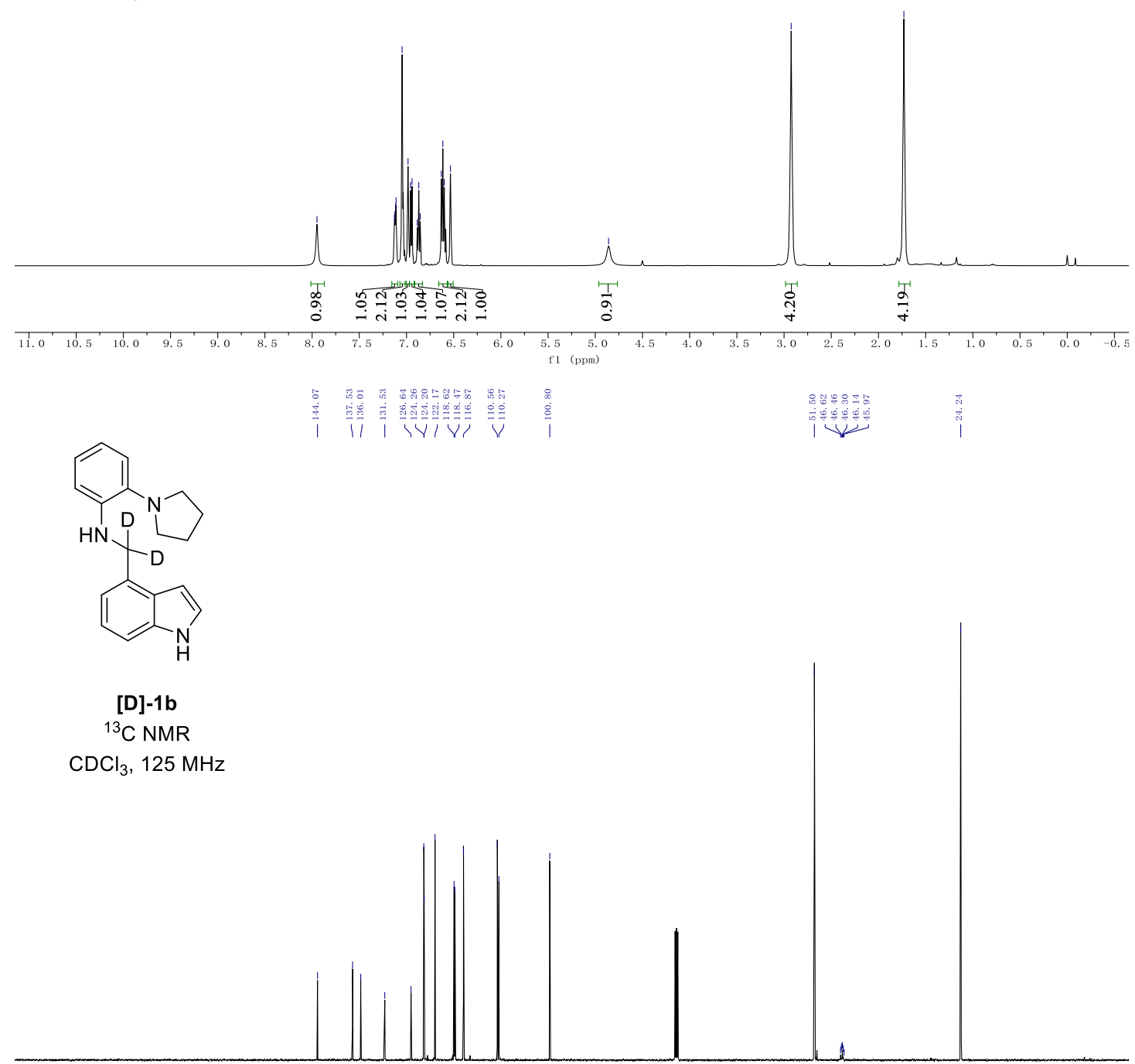

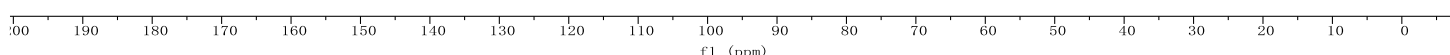



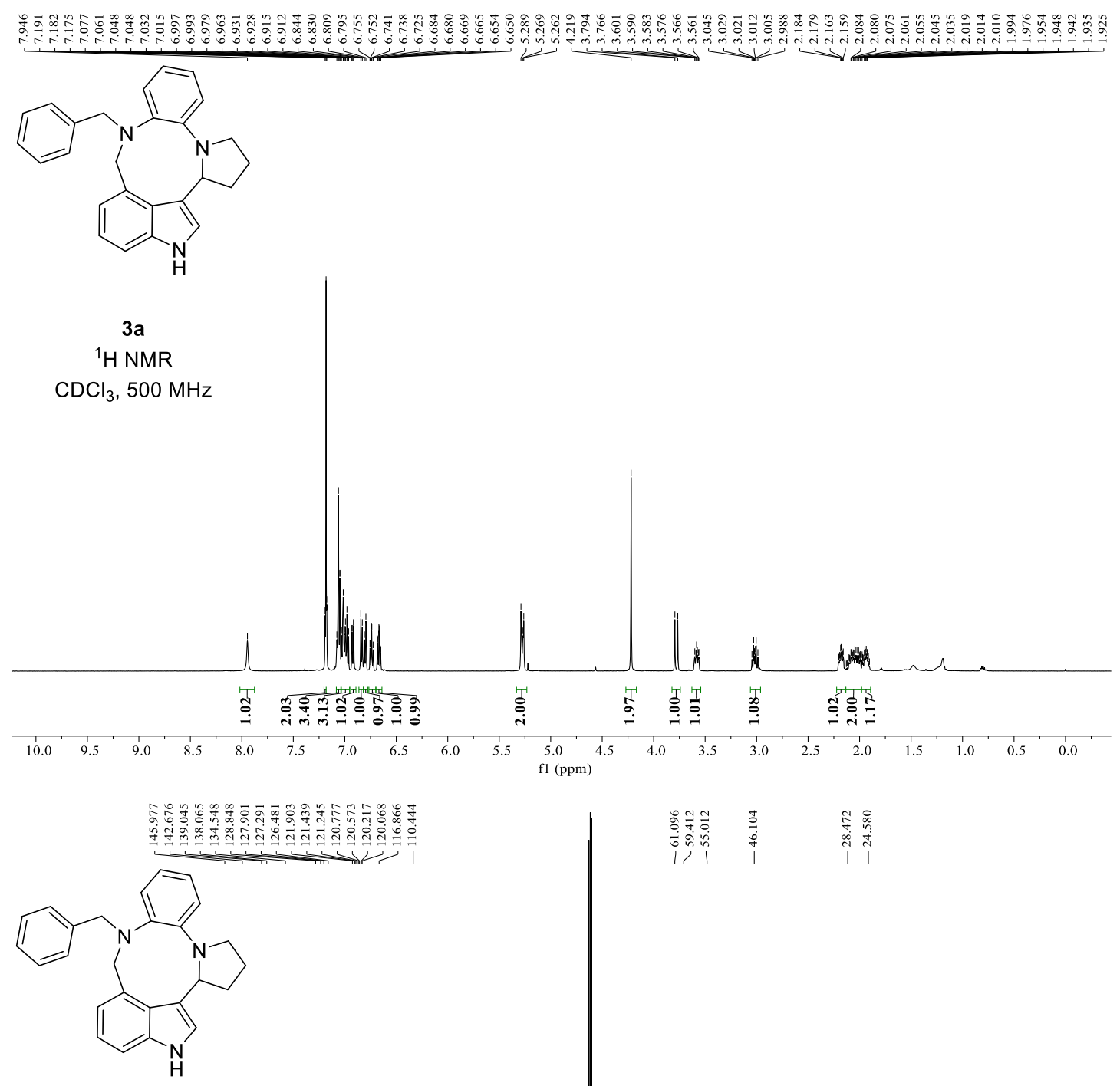

$3 \mathbf{a}$

${ }^{13} \mathrm{C}$ NMR

$\mathrm{CDCl}_{3}, 125 \mathrm{MHz}$

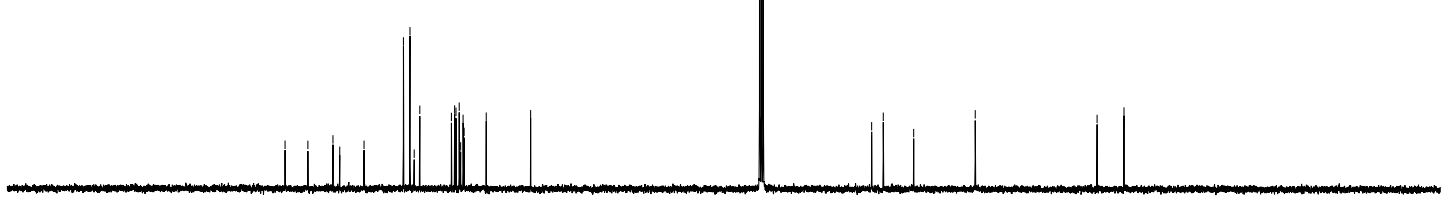

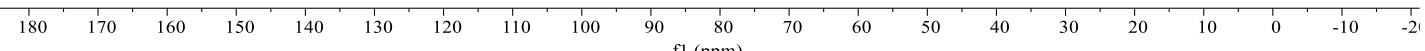


<smiles>O=[N+]([O-])c1ccc(CN2Cc3cccc4[nH]cc(c34)C3CCCN3c3ccccc32)cc1</smiles>

3b

${ }^{1} \mathrm{H}$ NMR $\mathrm{CDCl}_{3}, 500 \mathrm{MHz}$

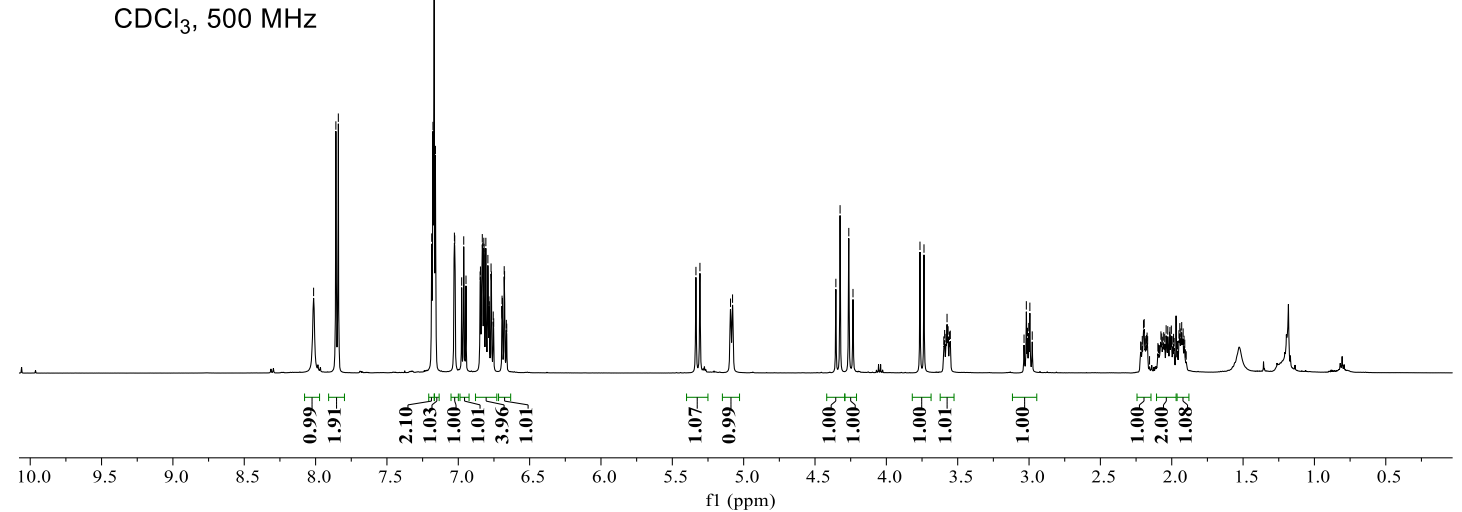

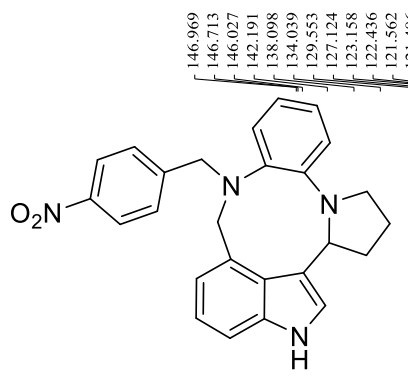

3b

${ }^{13} \mathrm{C}$ NMR $\mathrm{CDCl}_{3}, 125 \mathrm{MHz}$

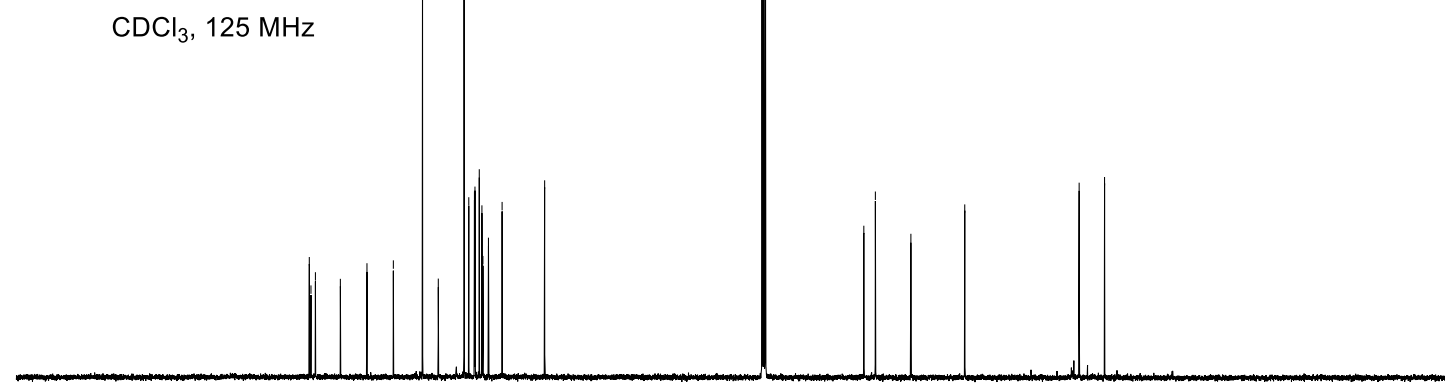

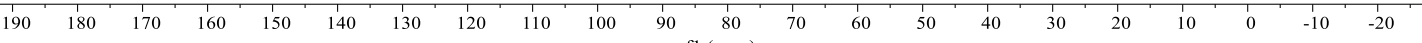


<smiles>N#Cc1ccc(CN2CCC3c4cccc2c(cc[nH]4)C2CCCN32)cc1</smiles>

$3 c$

${ }^{1} \mathrm{H}$ NMR

$\mathrm{CDCl}_{3}, 500 \mathrm{MHz}$

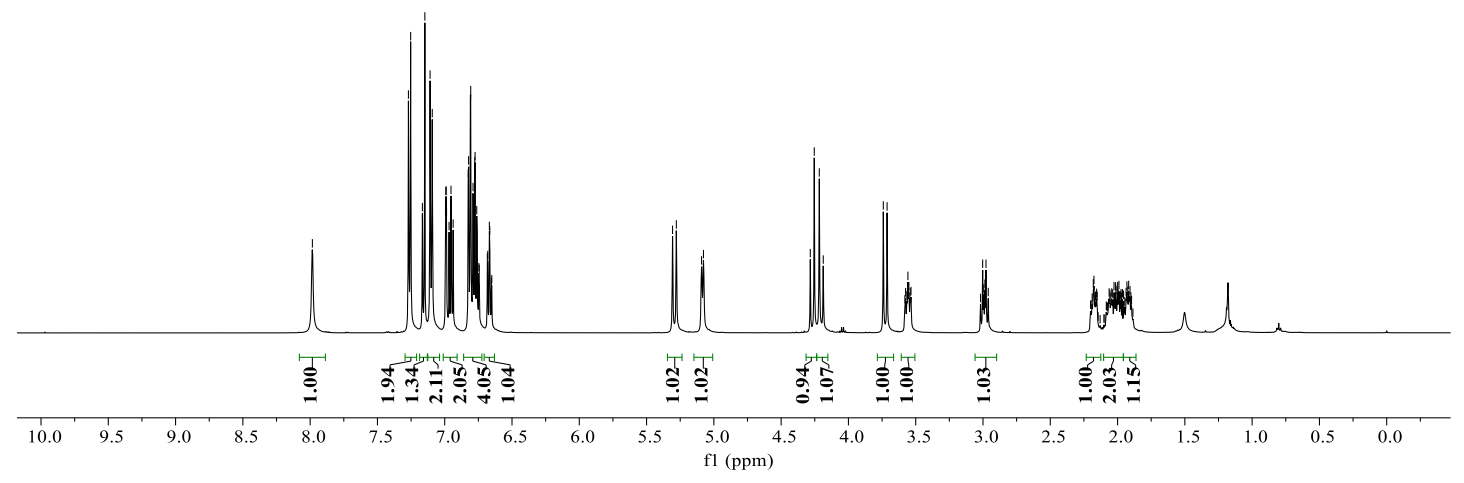

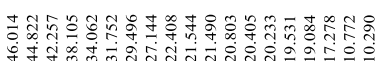

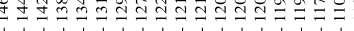

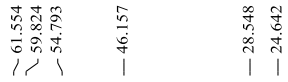<smiles>N#Cc1ccc(CN2Cc3cccc4[nH]cc(c34)C3CCCN3c3ccccc32)cc1</smiles>

$3 c$

${ }^{13} \mathrm{C}$ NMR $\mathrm{CDCl}_{3}, 125 \mathrm{MHz}$

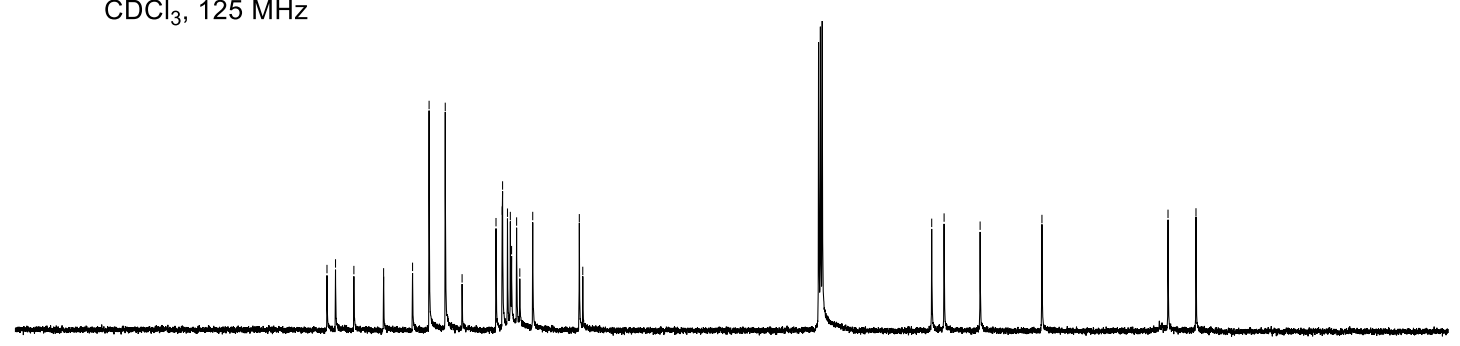

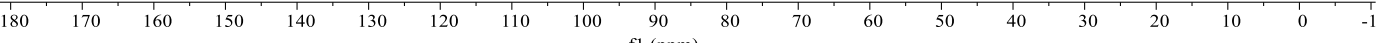




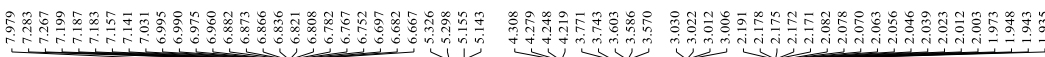<smiles></smiles>

3d

${ }^{1} \mathrm{H}$ NMR

$\mathrm{CDCl}_{3}, 500 \mathrm{MHz}$

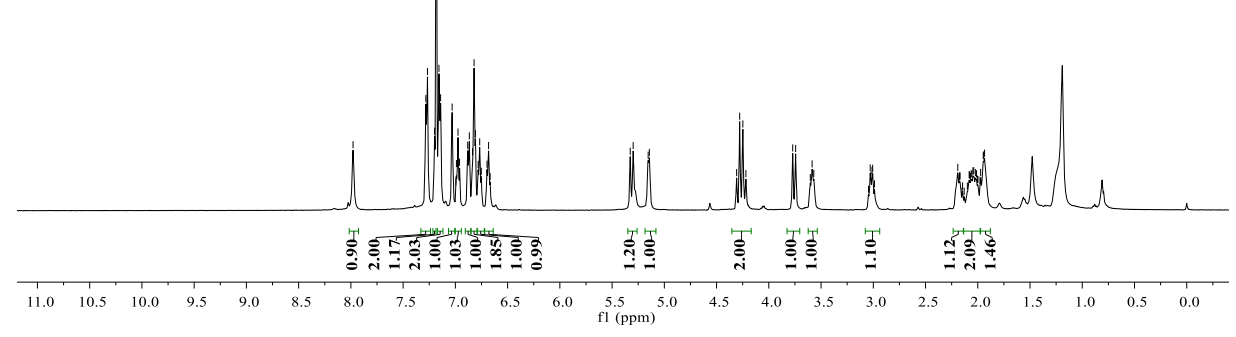

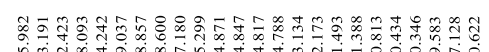

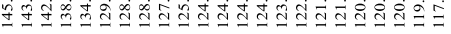

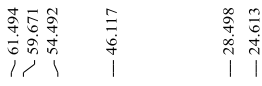<smiles>FC(F)(F)c1ccc(CN2Cc3cccc4[nH]cc(c34)C3CCCN3c3ccccc32)cc1</smiles>

3d

${ }^{13} \mathrm{C}$ NMR

$\mathrm{CDCl}_{3}, 125 \mathrm{MHz}$

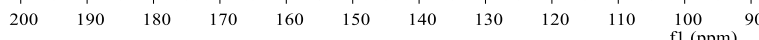


<smiles>FC(F)(F)c1ccc(CN2Cc3cccc4[nH]cc(c34)C3CCCN3c3ccccc32)cc1</smiles>

3d

${ }^{19} \mathrm{~F}$ NMR

$\mathrm{CDCl}_{3}, 470 \mathrm{MHz}$

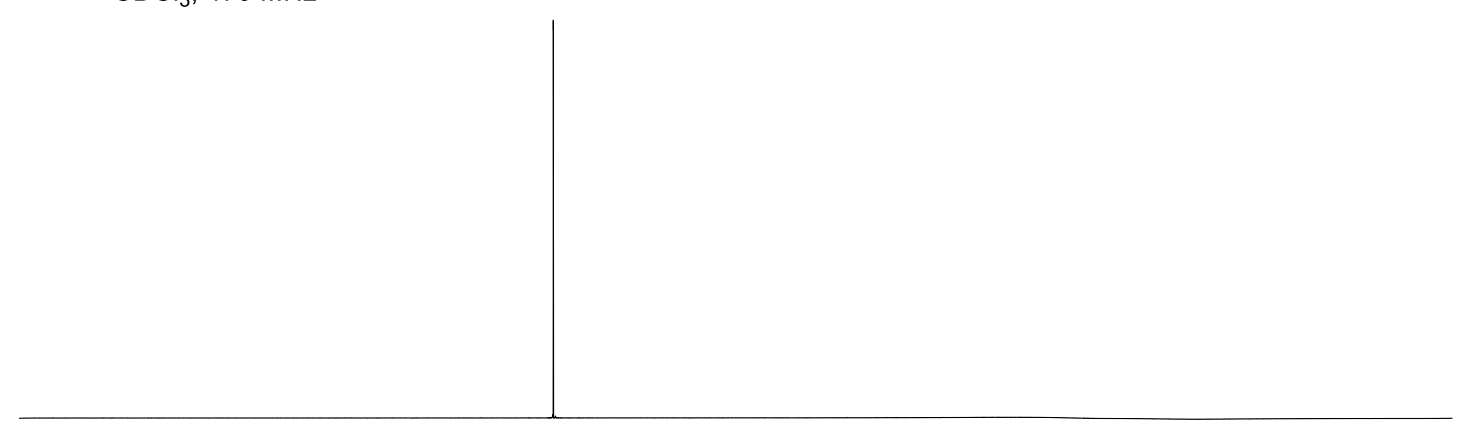

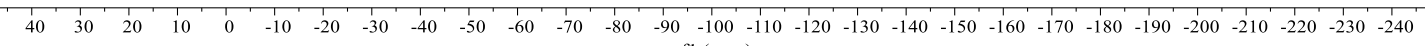
$\mathrm{fl}(\mathrm{ppm})$ 


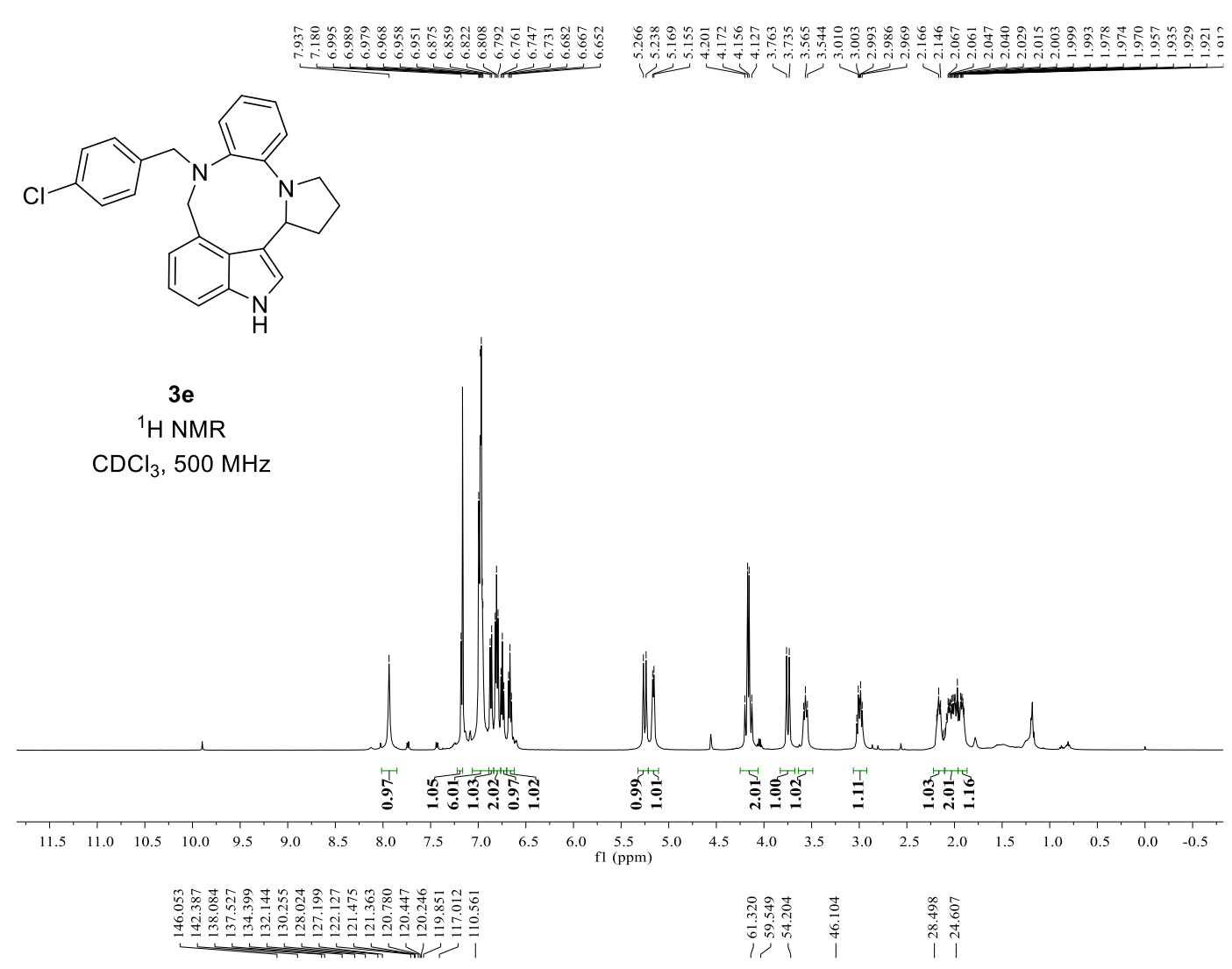<smiles>Clc1ccc(CN2Cc3cccc4[nH]cc(c34)C3CCCN3c3ccccc32)cc1</smiles>

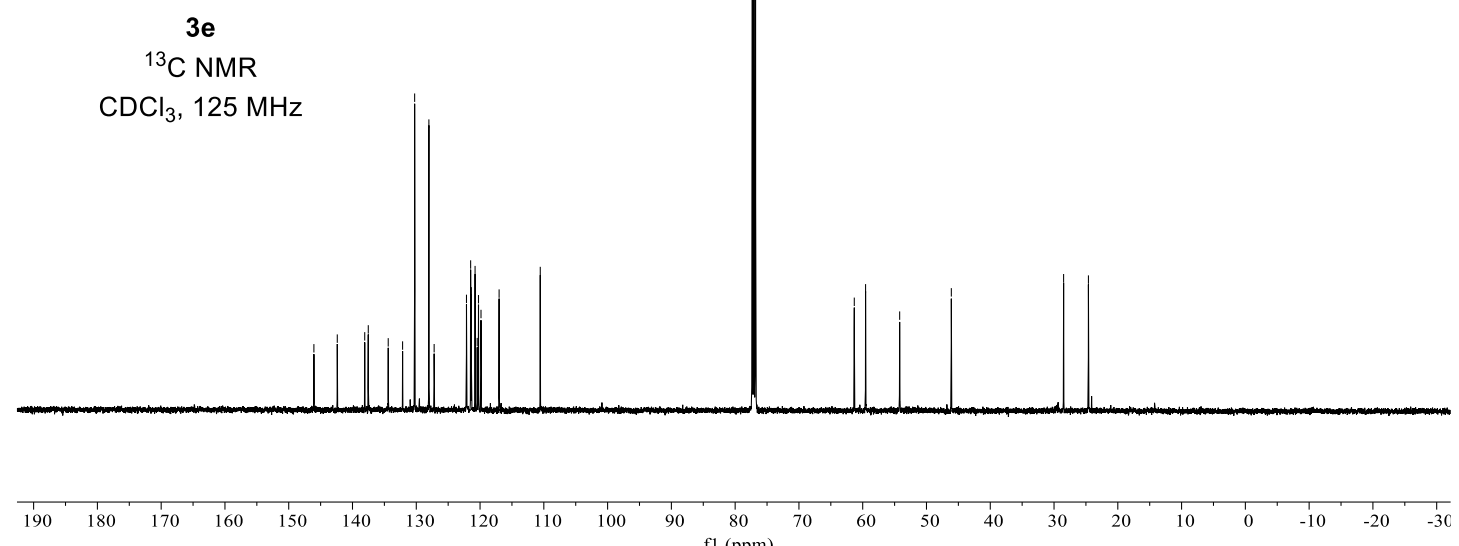


<smiles>COc1ccc(CN2Cc3cccc4[nH]cc(c34)C3CCCN3c3ccccc32)cc1</smiles>

3f

${ }^{1} \mathrm{H}$ NMR $\mathrm{CDCl}_{3}, 500 \mathrm{MHz}$

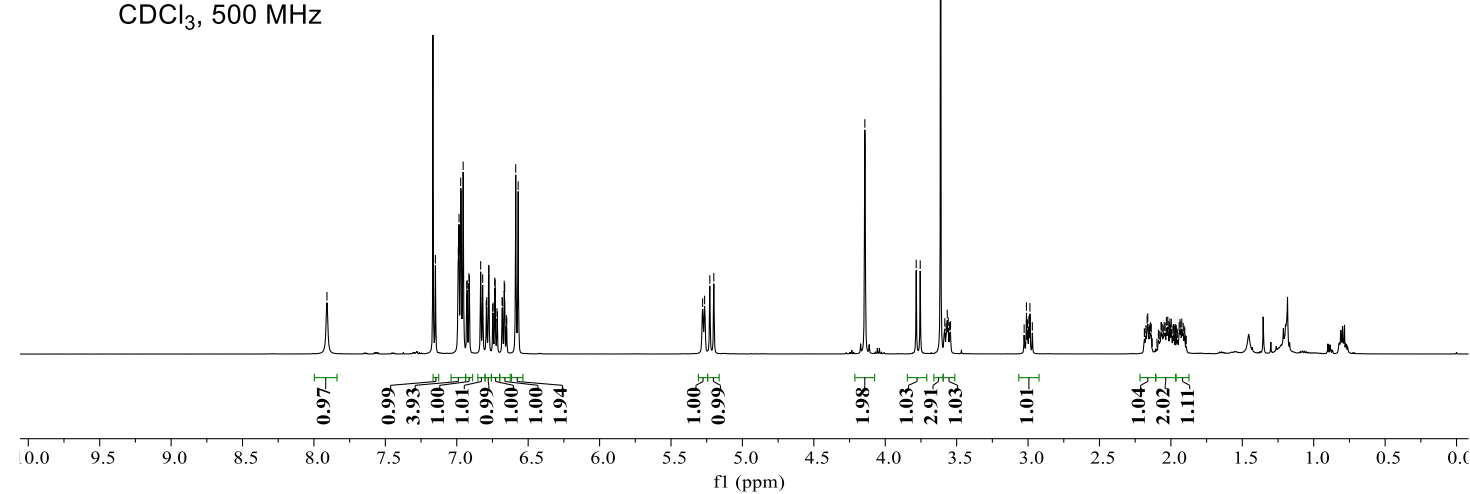

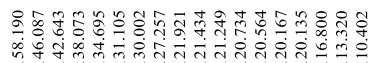

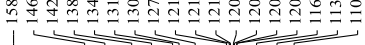<smiles>COc1ccc(CN2Cc3cccc4[nH]cc(c34)C3CCCN3c3ccccc32)cc1</smiles>

$3 f$

${ }^{13} \mathrm{C}$ NMR

$\mathrm{CDCl}_{3}, 125 \mathrm{MHz}$

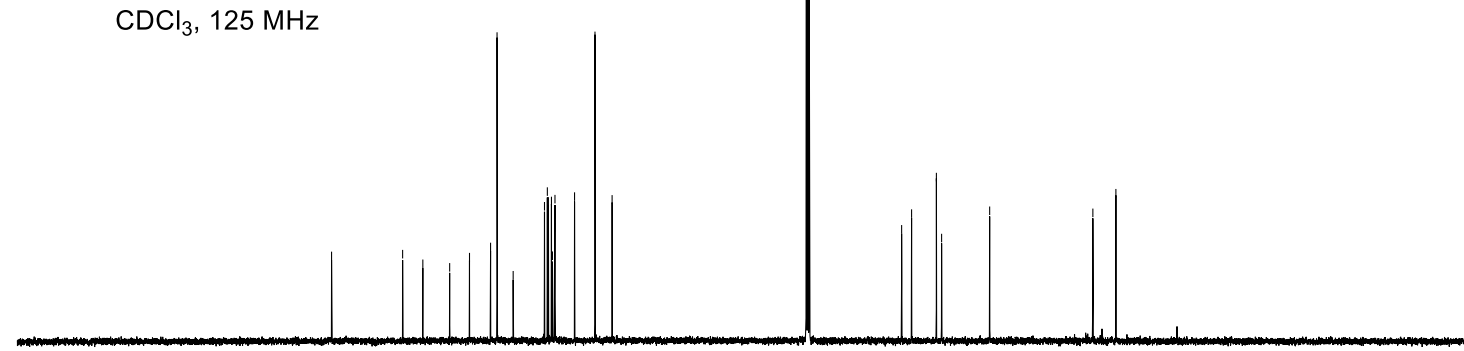

\begin{tabular}{lllllllllllllllllllllllllll}
\hline & 1 \\
210 & 200 & 190 & 180 & 170 & 160 & 150 & 140 & 130 & 120 & 110 & 100 & 90 & 80 & 70 & 60 & 50 & 40 & 30 & 20 & 10 & 0 & -10 & -20 & -30
\end{tabular} 


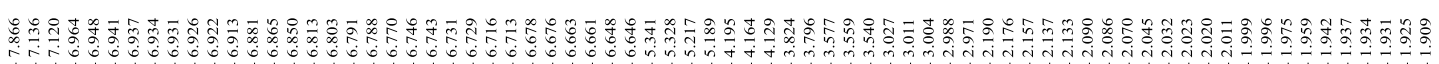<smiles>Cc1cccc(CN2Cc3cccc4[nH]cc(c34)C3CCCN3c3ccccc32)c1</smiles>

$3 g$

${ }^{1} \mathrm{H}$ NMR

$\mathrm{CDCl}_{3}, 500 \mathrm{MHz}$
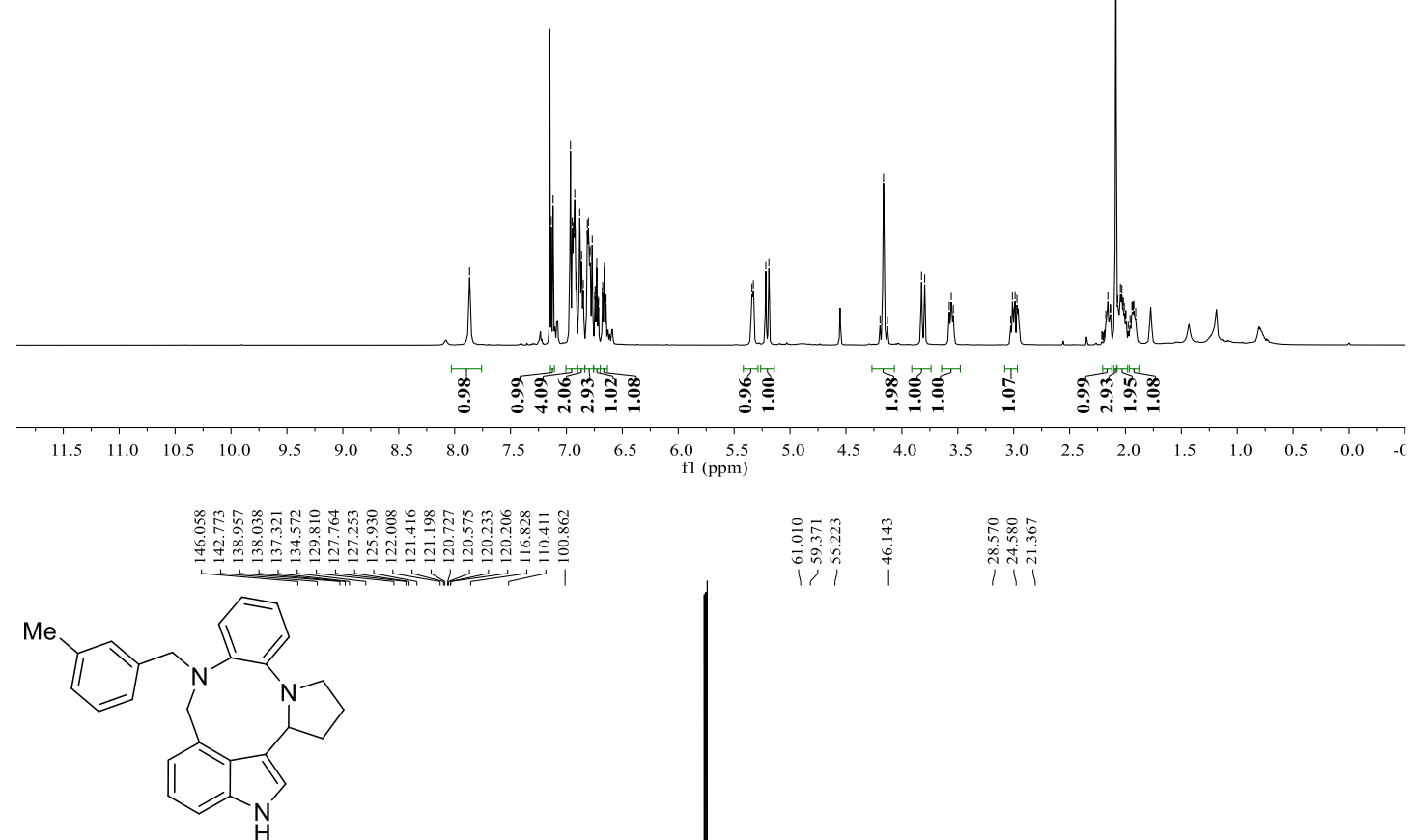

$3 g$

${ }^{13} \mathrm{C}$ NMR

$\mathrm{CDCl}_{3}, 125 \mathrm{MHz}$

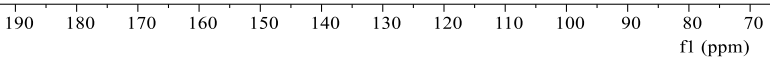

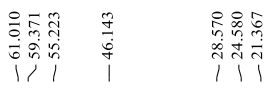

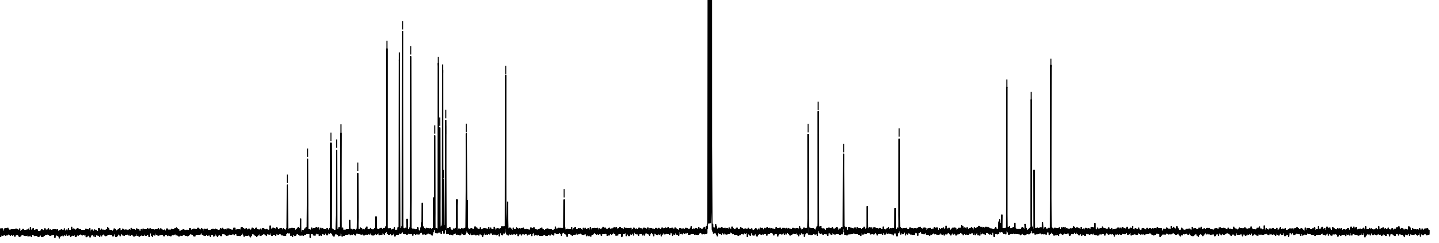




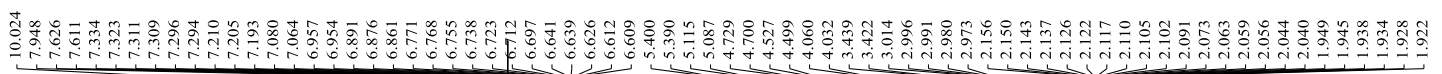<smiles>Cc1ccccc1CN1Cc2cccc3[nH]cc(c23)C2CCCN2c2ccccc21</smiles>

$3 \mathrm{~h}$

${ }^{1} \mathrm{H}$ NMR

$\mathrm{CDCl}_{3}, 500 \mathrm{MHz}$

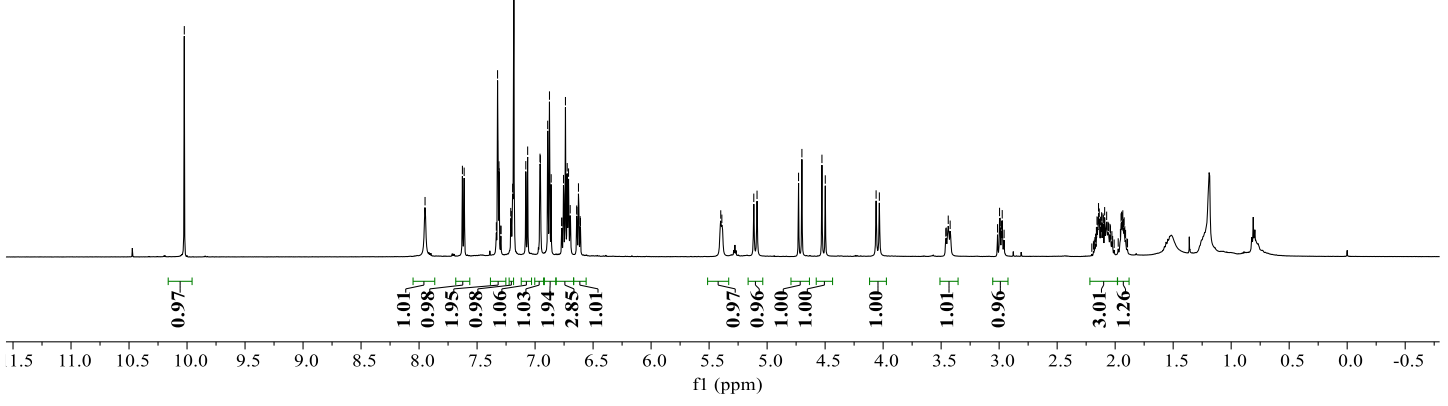<smiles>O=Cc1ccccc1CN1Cc2cccc3[nH]cc(c23)C2CCCN2c2ccccc21</smiles>

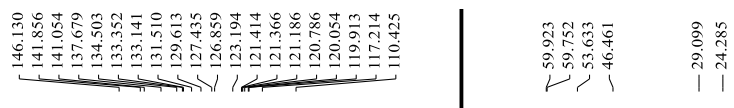

$3 \mathrm{~h}$

${ }^{13} \mathrm{C}$ NMR

$\mathrm{CDCl}_{3}, 125 \mathrm{MHz}$

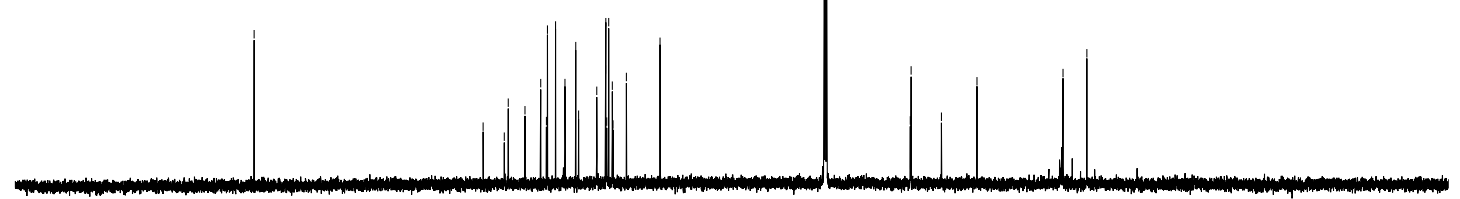

\begin{tabular}{llllllllllllllllllllllllllllll}
\hline 40 & 230 & 220 & 210 & 200 & 190 & 180 & 170 & 160 & 150 & 140 & 130 & 120 & 110 & 100 & 90 & 80 & 70 & 60 & 50 & 40 & 30 & 20 & 10 & 0 & -10 & -20 & -30 & -40
\end{tabular} 
<smiles>c1coc(CN2Cc3cccc4[nH]cc(c34)C3CCCN3c3ccccc32)c1</smiles>

$3 \mathbf{i}$

${ }^{1} \mathrm{H}$ NMR

$\mathrm{CDCl}_{3}, 500 \mathrm{MHz}$

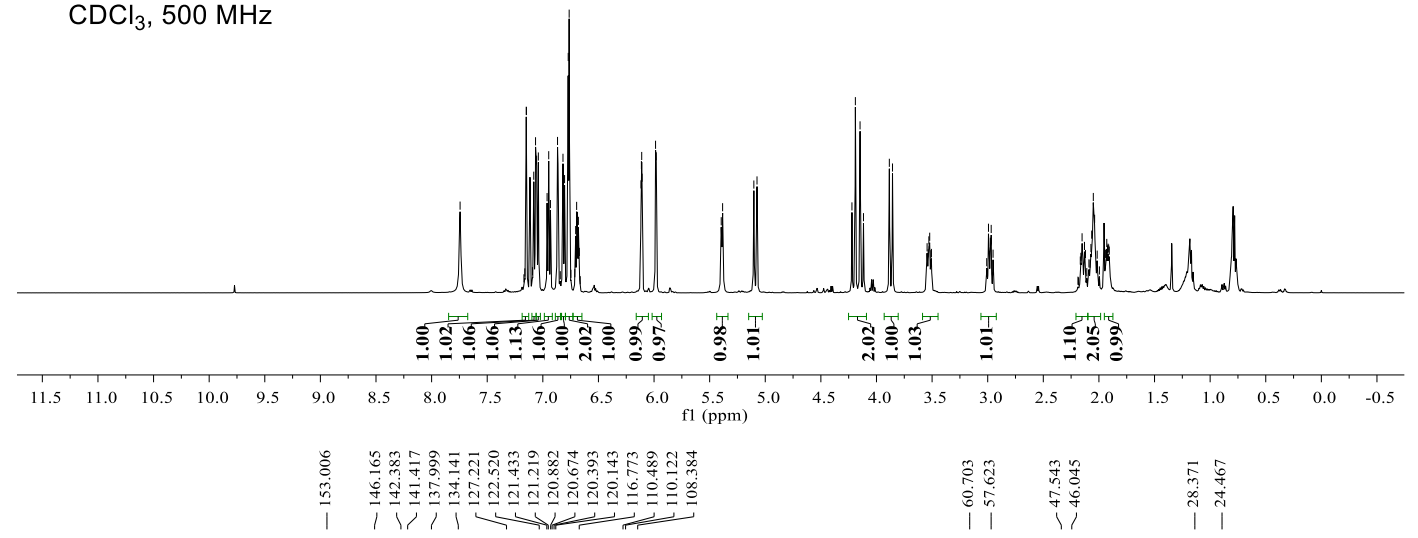<smiles>c1coc(CN2Cc3cccc4[nH]cc(c34)C3CCCN3c3ccccc32)c1</smiles>

$3 \mathbf{i}$

${ }^{13} \mathrm{C}$ NMR

$\mathrm{CDCl}_{3}, 125 \mathrm{MHz}$

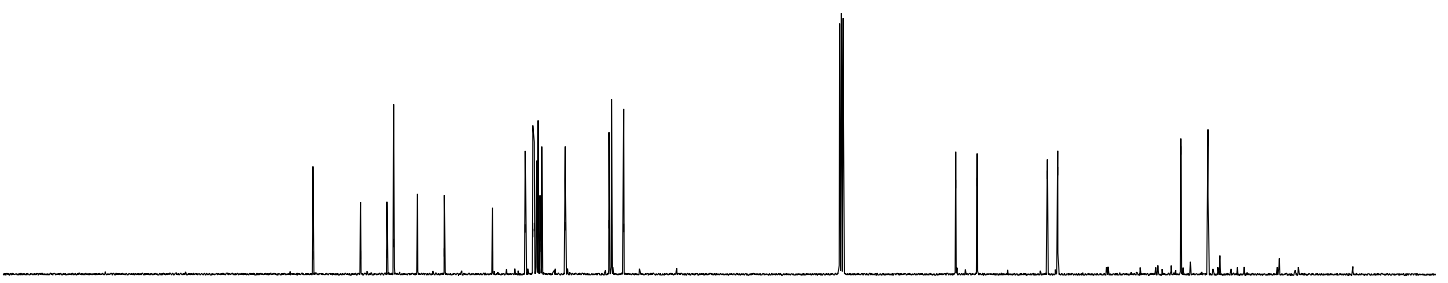

$\begin{array}{llllllllll}190 & 180 & 170 & 160 & 150 & 140 & 130 & 120 & 110 & \begin{array}{c}100 \\ \mathrm{f} 1(\mathrm{ppm})\end{array}\end{array}$ 
<smiles>c1csc(CN2Cc3cccc4[nH]cc(c34)C3CCCN3c3ccccc32)c1</smiles>

3j

${ }^{1} \mathrm{H}$ NMR

$\mathrm{CDCl}_{3}, 500 \mathrm{MHz}$

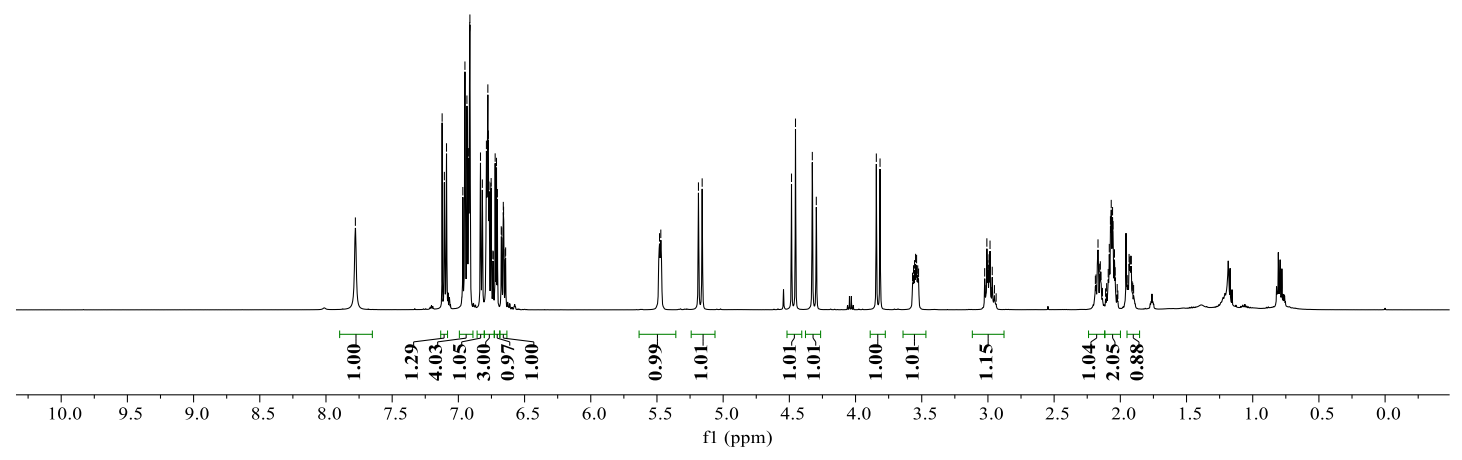

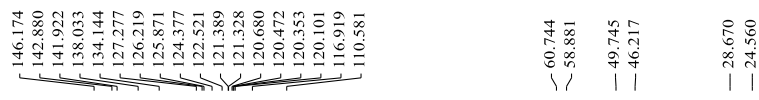

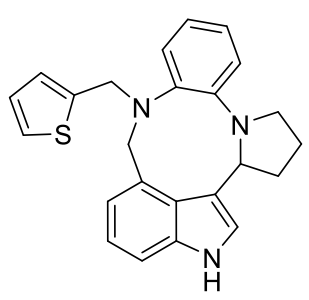

$3 \mathbf{j}$

${ }^{13} \mathrm{C}$ NMR

$\mathrm{CDCl}_{3}, 125 \mathrm{MHz}$

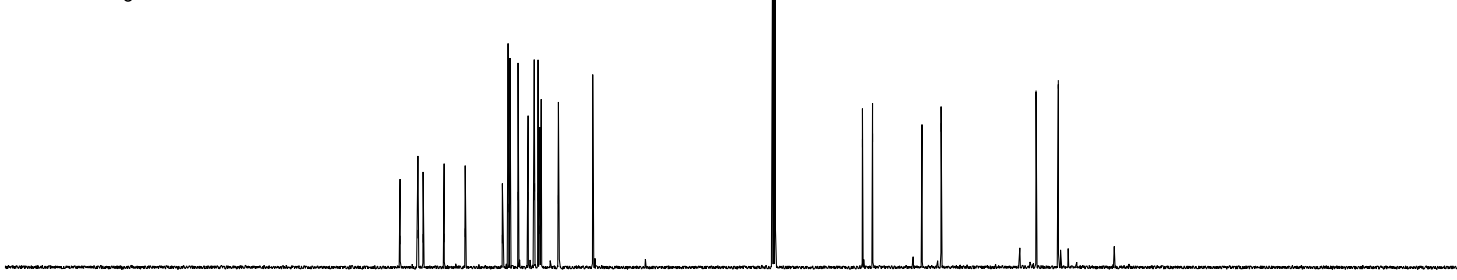

$\begin{array}{llllllllllllllllllllllllllllll} & 210 & 200 & 190 & 180 & 170 & 160 & 150 & 140 & 130 & 120 & 110 & 100 & 90 & 80 & 70 & 60 & 50 & 40 & 30 & 20 & 10 & 0 & -10 & -20 & -30 & -40\end{array}$ 
<smiles>NC=c1c2c[nH]c3cccc(c32)n(C/C=C/c2ccccc2)c2ccccc12</smiles>

3k

${ }^{1} \mathrm{H}$ NMR

$\mathrm{CDCl}_{3}, 500 \mathrm{MHz}$

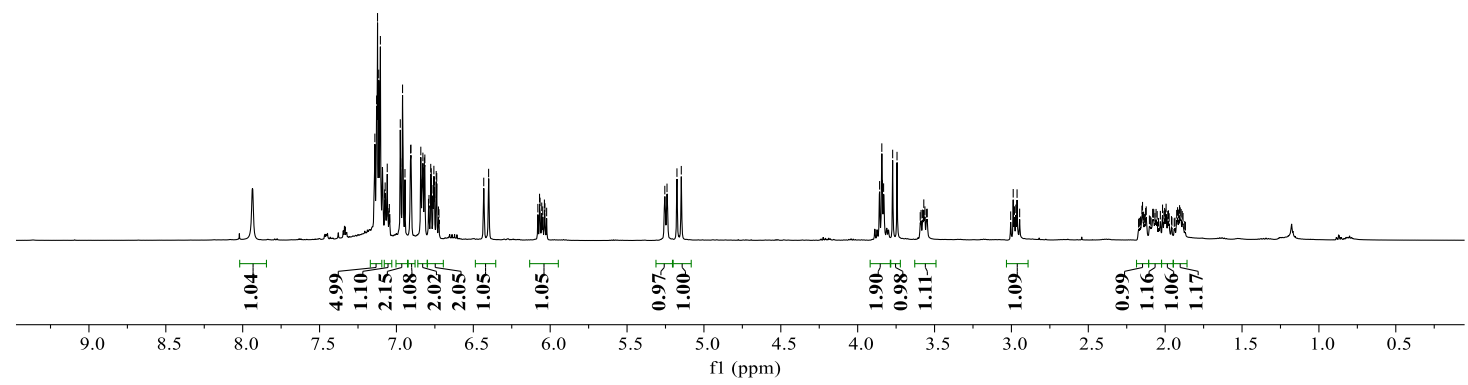

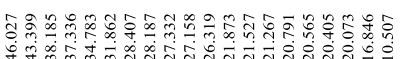

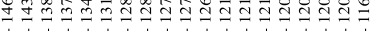<smiles>C(=C/c1ccccc1)\CN1Cc2cccc3[nH]cc(c23)C2CCCN2c2ccccc21</smiles>

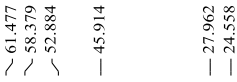

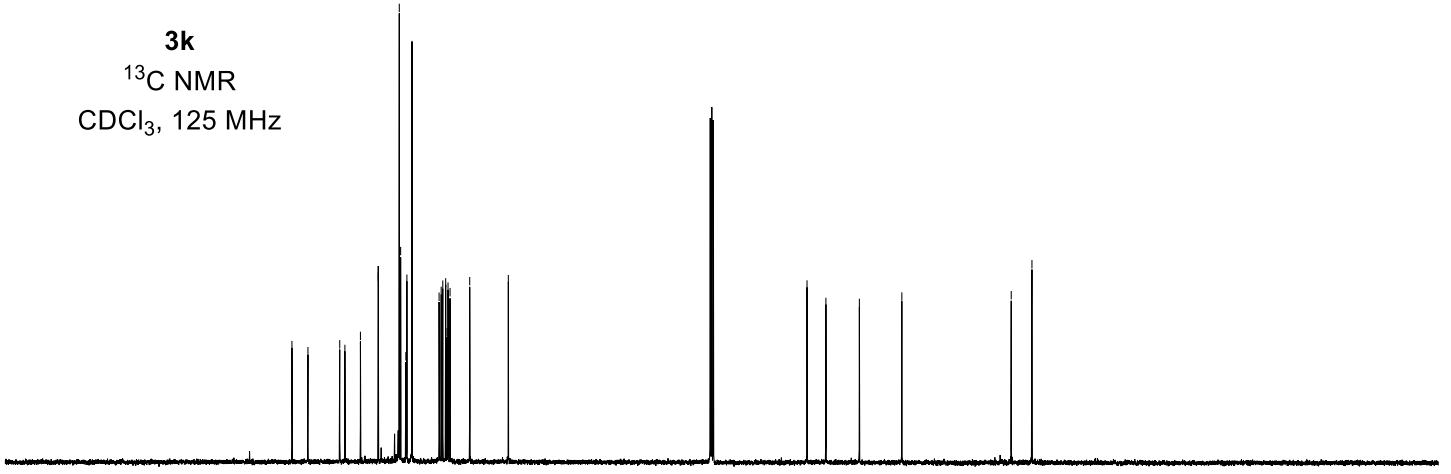

$\begin{array}{lllllllllllllllllllllllll}190 & 180 & 170 & 160 & 150 & 140 & 130 & 120 & 110 & 100 & 90 & 80 & 70 & 60 & 50 & 40 & 30 & 20 & 10 & 0 & -10 & -20 & -30 & -4 \mathrm{C}\end{array}$ 


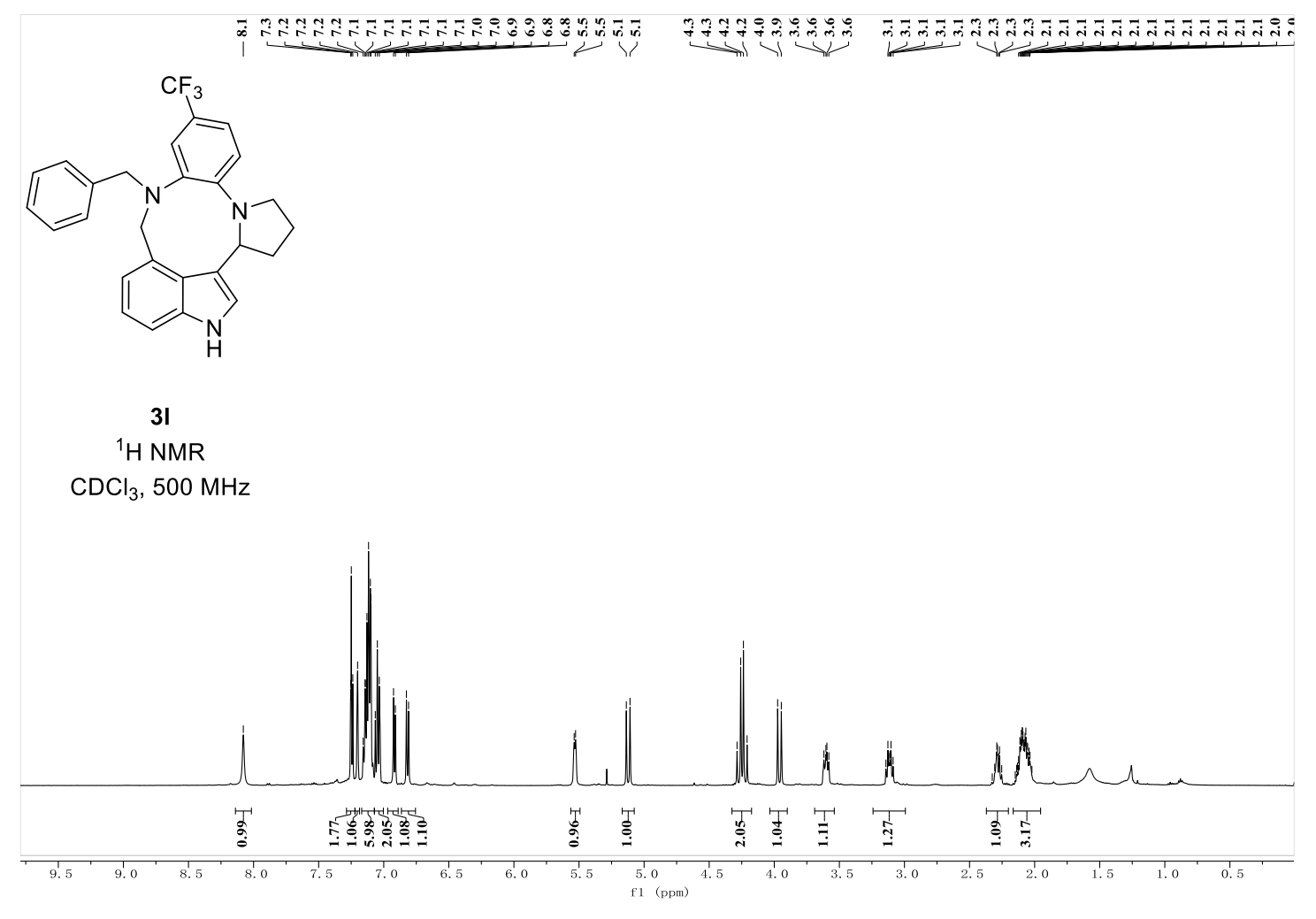

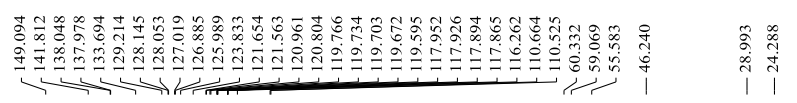<smiles>FC(F)(F)c1ccc2c(c1)N(Cc1ccccc1)Cc1cccc3[nH]cc(c13)C1CCCN21</smiles>

3I

${ }^{13} \mathrm{C}$ NMR

$\mathrm{CDCl}_{3}, 125 \mathrm{MHz}$

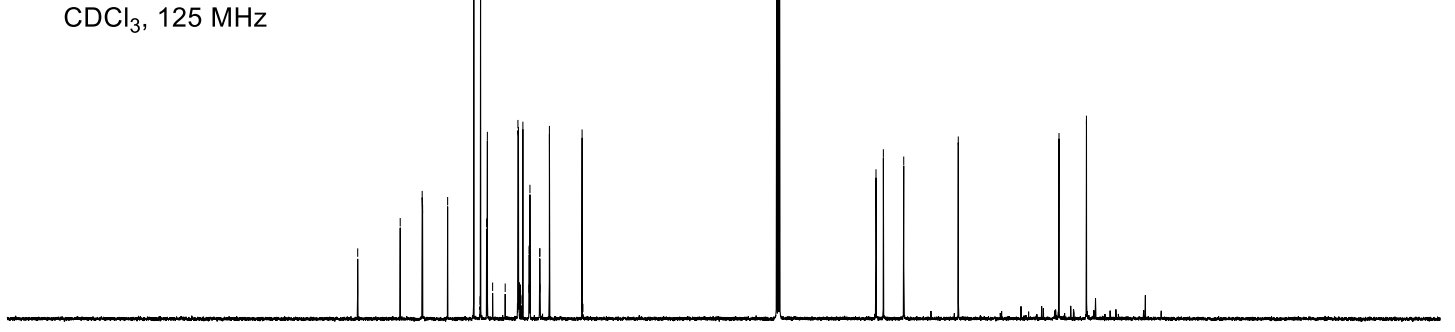

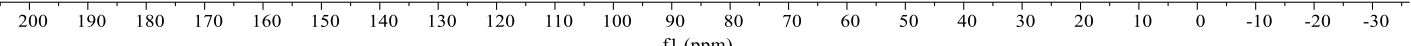




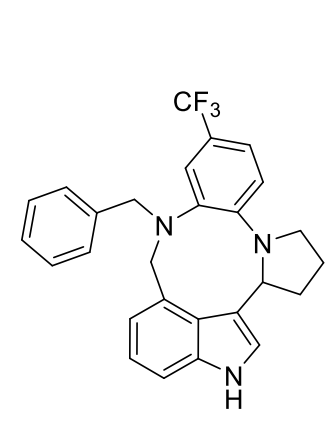

31

${ }^{19} \mathrm{~F} \mathrm{NMR}$

$\mathrm{CDCl}_{3}, 470 \mathrm{MHz}$

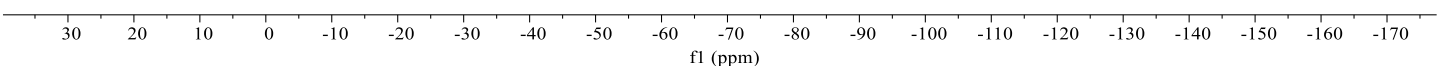


<smiles>N#Cc1ccc2c(c1)N(Cc1ccccc1)Cc1cccc3[nH]cc(c13)C1CCCN21</smiles>

$3 m$

${ }^{1} \mathrm{H}$ NMR

$\mathrm{CDCl}_{3}, 500 \mathrm{MHz}$

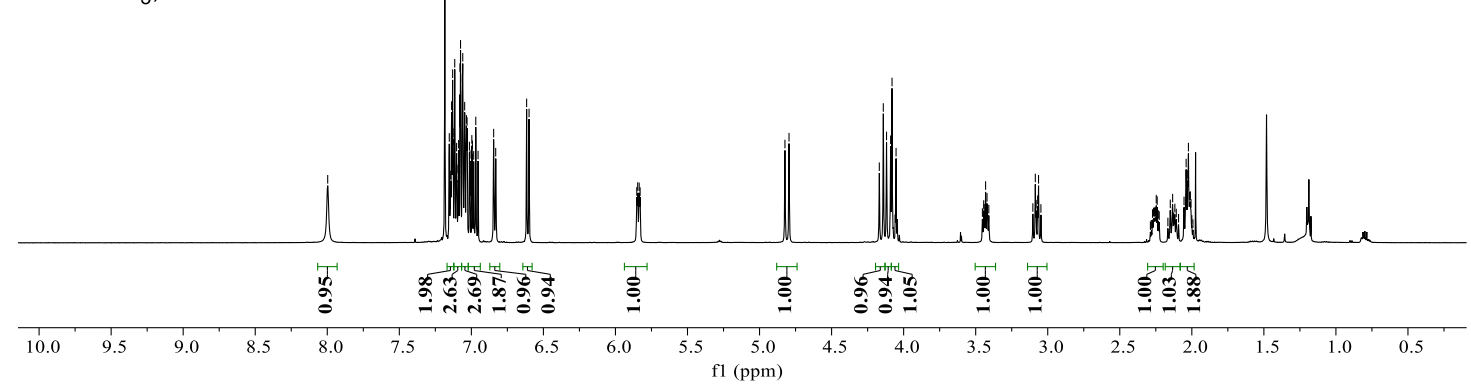

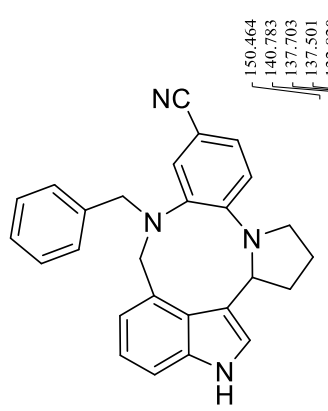

$3 \mathrm{~m}$

${ }^{13} \mathrm{C}$ NMR

$\mathrm{CDCl}_{3}, 125 \mathrm{MHz}$

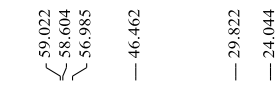

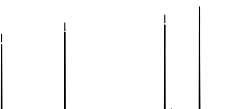

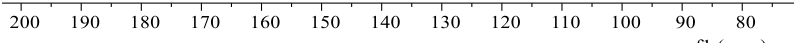

$\begin{array}{llllllllll}50 & 40 & 30 & 20 & 10 & 0 & -10 & -20 & -30\end{array}$ 


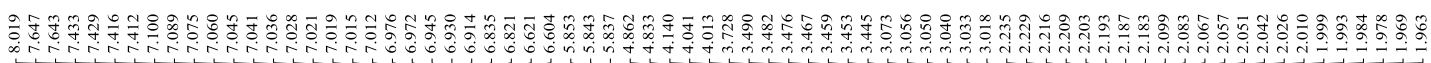

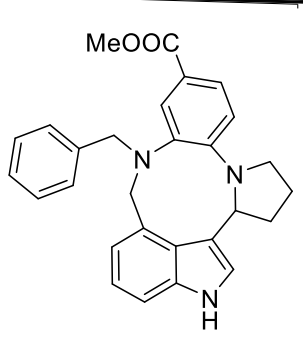

3n

${ }^{1} \mathrm{H}$ NMR $\mathrm{CDCl}_{3}, 500 \mathrm{MHz}$

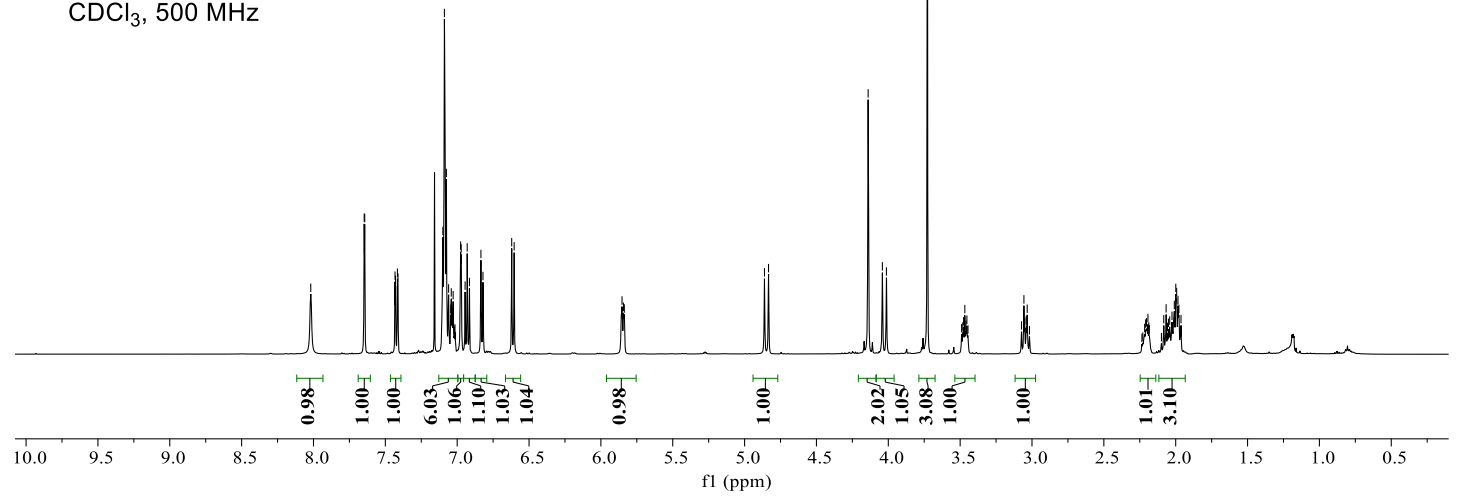

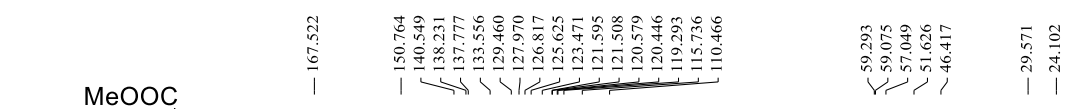<smiles>COc1ccc2c(c1)N(Cc1ccccc1)Cc1cccc3[nH]cc(c13)C1CCCN21</smiles>

3n

${ }^{13} \mathrm{C}$ NMR $\mathrm{CDCl}_{3}, 125 \mathrm{MHz}$

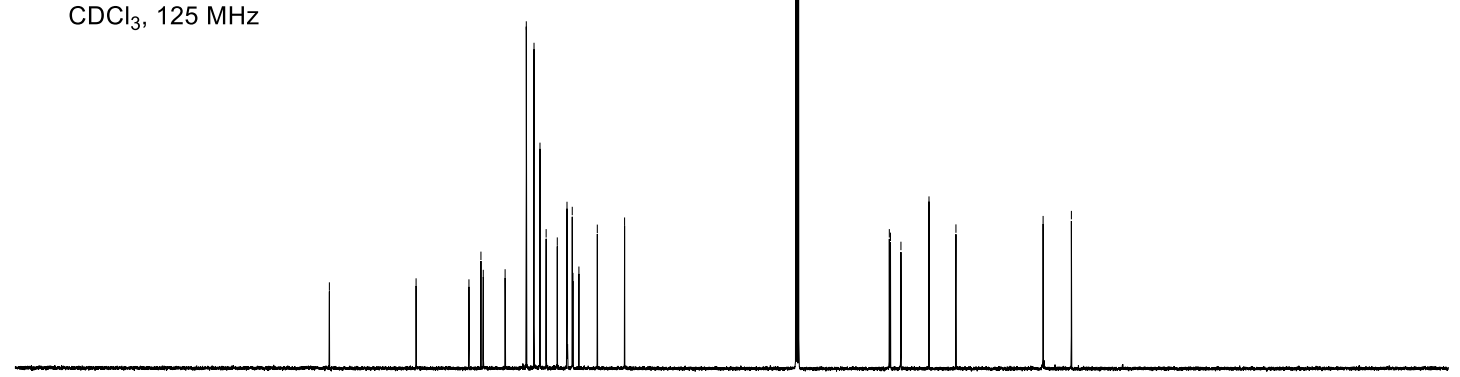

$\begin{array}{rllllllllllllllllllllllllll}220 & 210 & 200 & 190 & 180 & 170 & 160 & 150 & 140 & 130 & 120 & 110 & 100 & 90 & 80 & 70 & 60 & 50 & 40 & 30 & 20 & 10 & 0 & -10 & -20 & -30 & -40\end{array}$ 


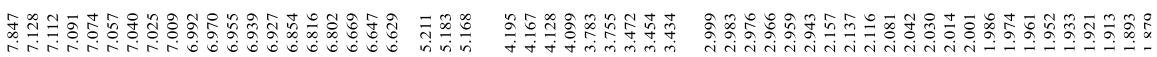<smiles>Clc1ccc2c(c1)N(Cc1ccccc1)Cc1cccc3[nH]cc(c13)C1CCCN21</smiles>

30

${ }^{1} \mathrm{H}$ NMR

$\mathrm{CDCl}_{3}, 500 \mathrm{MHz}$

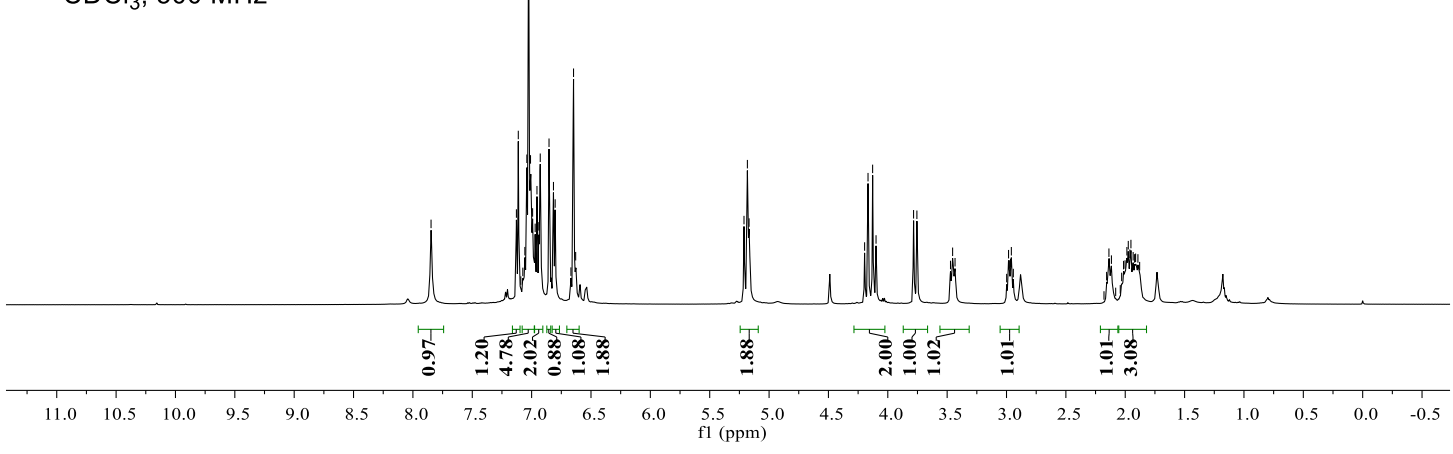

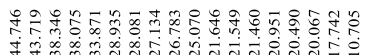

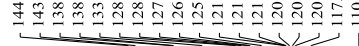<smiles>Clc1ccc2c(c1)N(Cc1ccccc1)Cc1cccc3[nH]cc(c13)C1CCCN21</smiles>

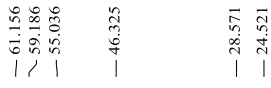

30

${ }^{13} \mathrm{C}$ NMR

$\mathrm{CDCl}_{3}, 125 \mathrm{MHz}$

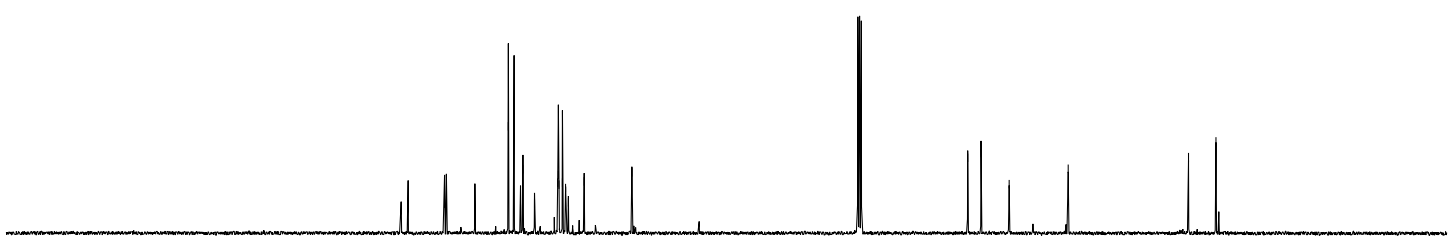

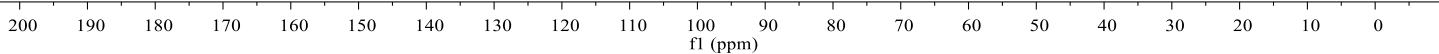



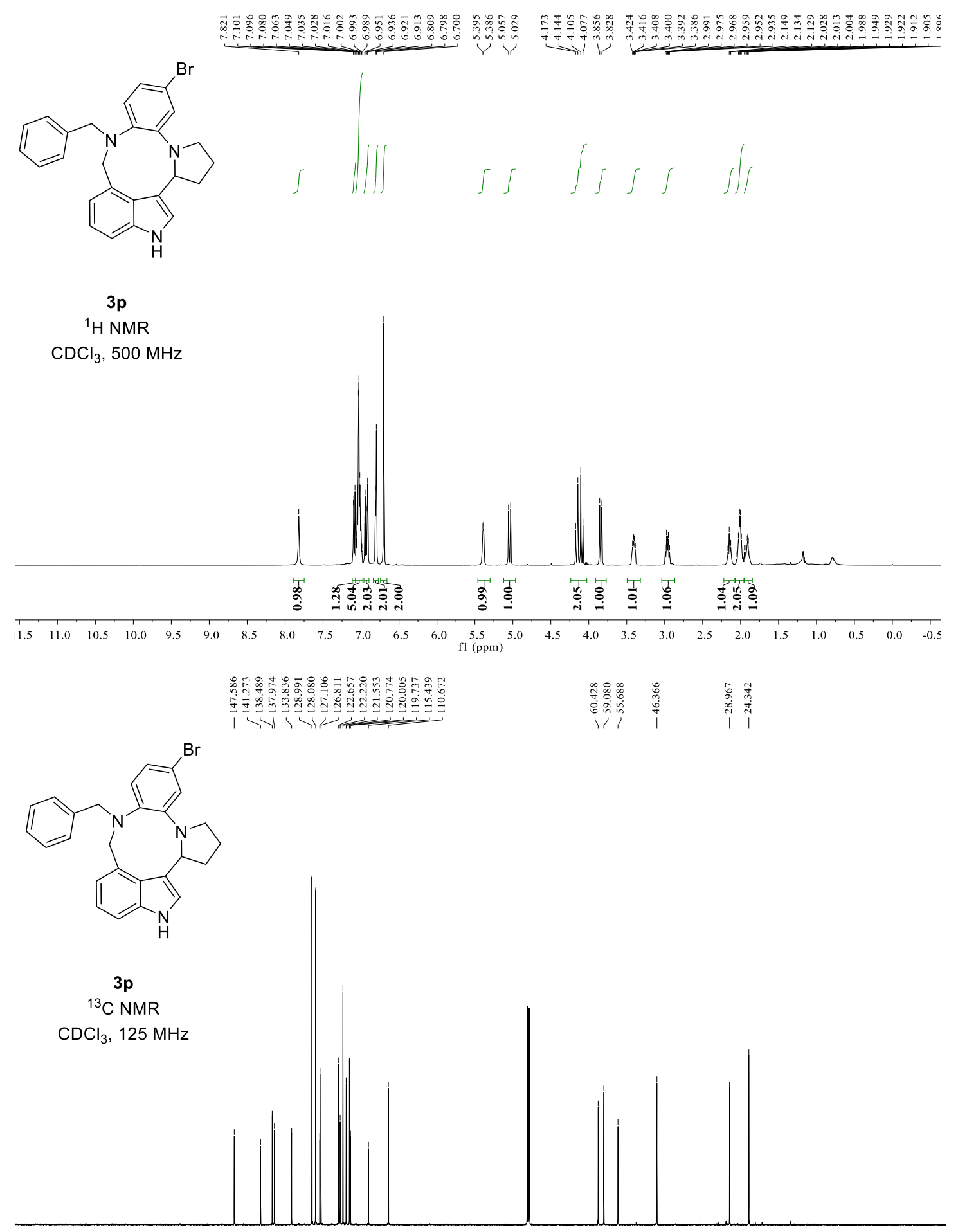

\begin{tabular}{llllllllllllllllllllllll}
\hline 00 & 190 & 180 & 170 & 160 & 150 & 140 & 130 & 120 & 110 & 100 & 90 & 80 & 70 & 60 & 50 & 40 & 30 & 20 & 10 & 0 & -10 & -20
\end{tabular} 

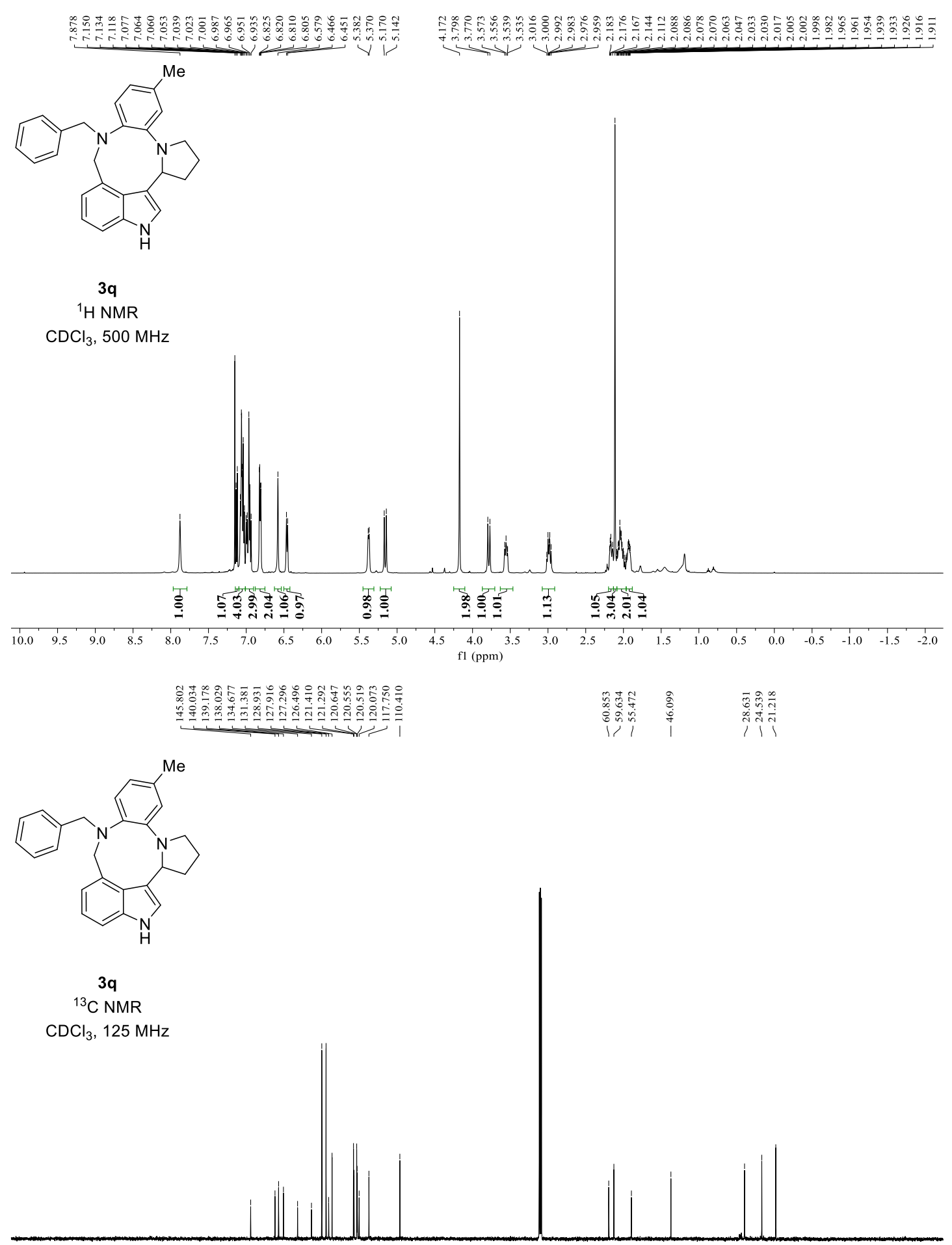

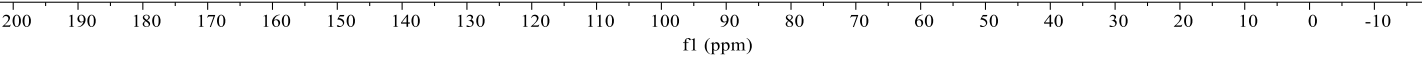




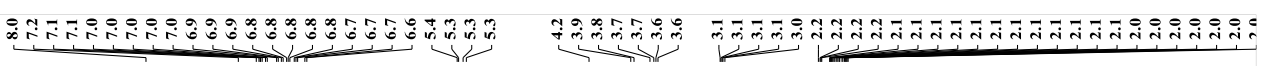<smiles>COc1ccc2c(c1)N(Cc1ccccc1)Cc1cccc3[nH]cc(c13)C1CCCN21</smiles>

$3 r$

${ }^{1} \mathrm{H}$ NMR $\mathrm{CDCl}_{3}, 500 \mathrm{MHz}$

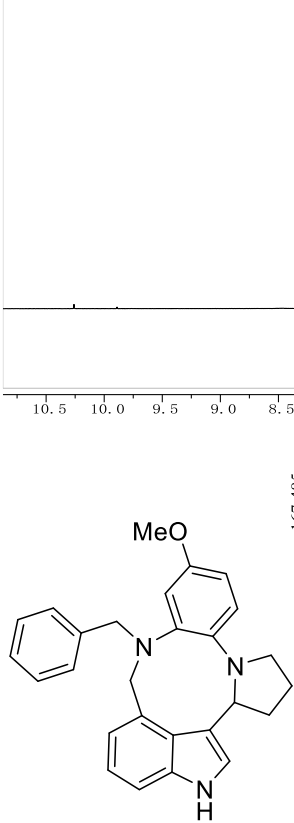

$3 r$

${ }^{13} \mathrm{C}$ NMR

$\mathrm{CDCl}_{3}, 125 \mathrm{MHz}$

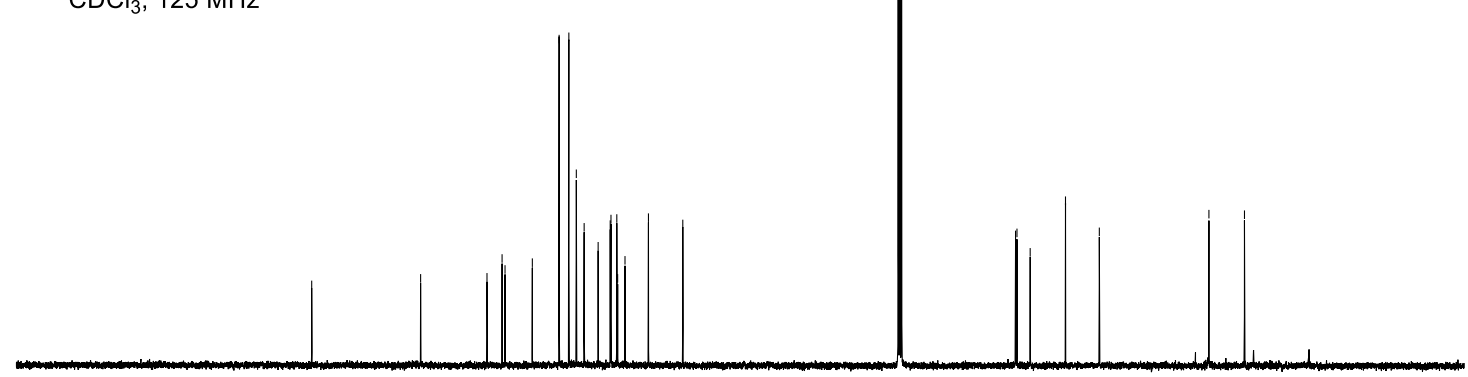

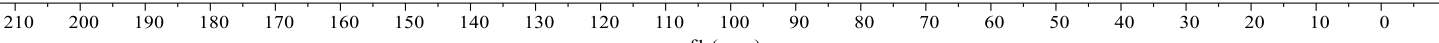


<smiles></smiles>

$3 s$

${ }^{1} \mathrm{H}$ NMR

$\mathrm{CDCl}_{3}, 500 \mathrm{MHz}$

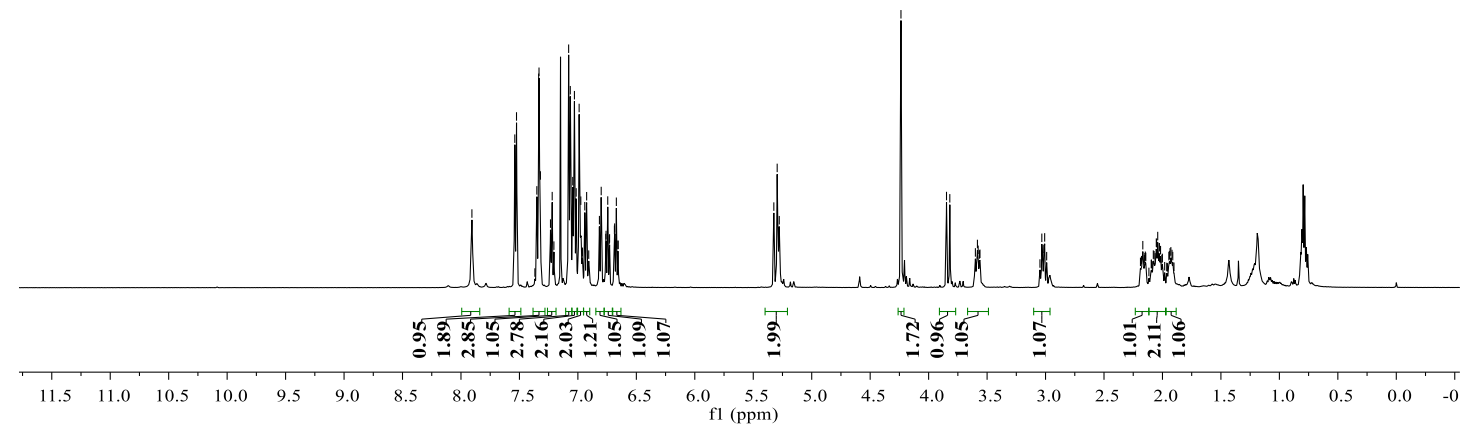

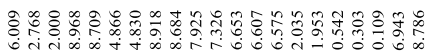

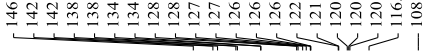

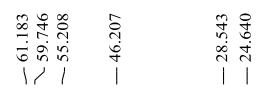<smiles>c1ccc(CN2Cc3cc(-c4ccccc4)cc4[nH]cc(c34)C3CCCN3c3ccccc32)cc1</smiles>

3s

${ }^{13} \mathrm{C}$ NMR

$\mathrm{CDCl}_{3}, 125 \mathrm{MHz}$

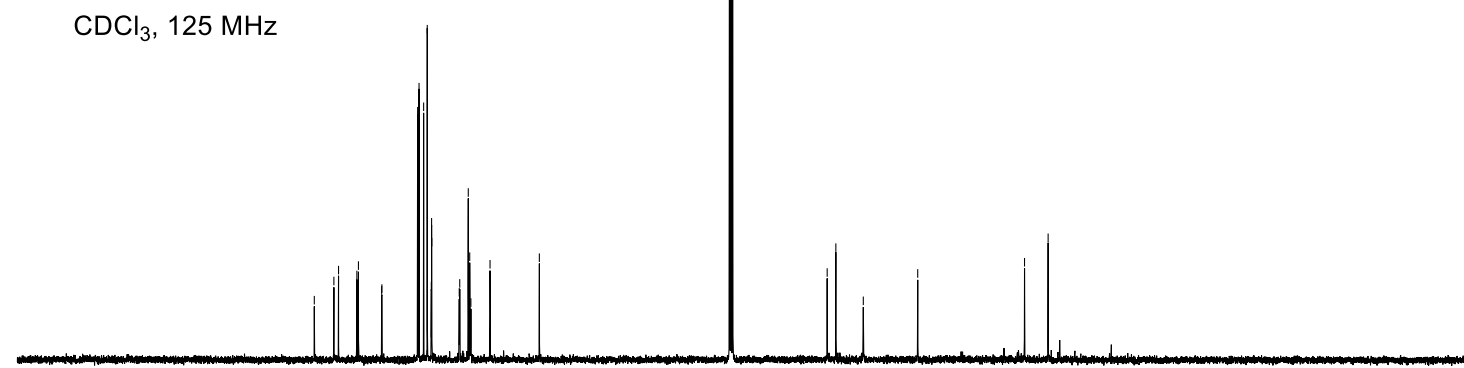

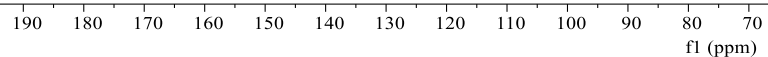



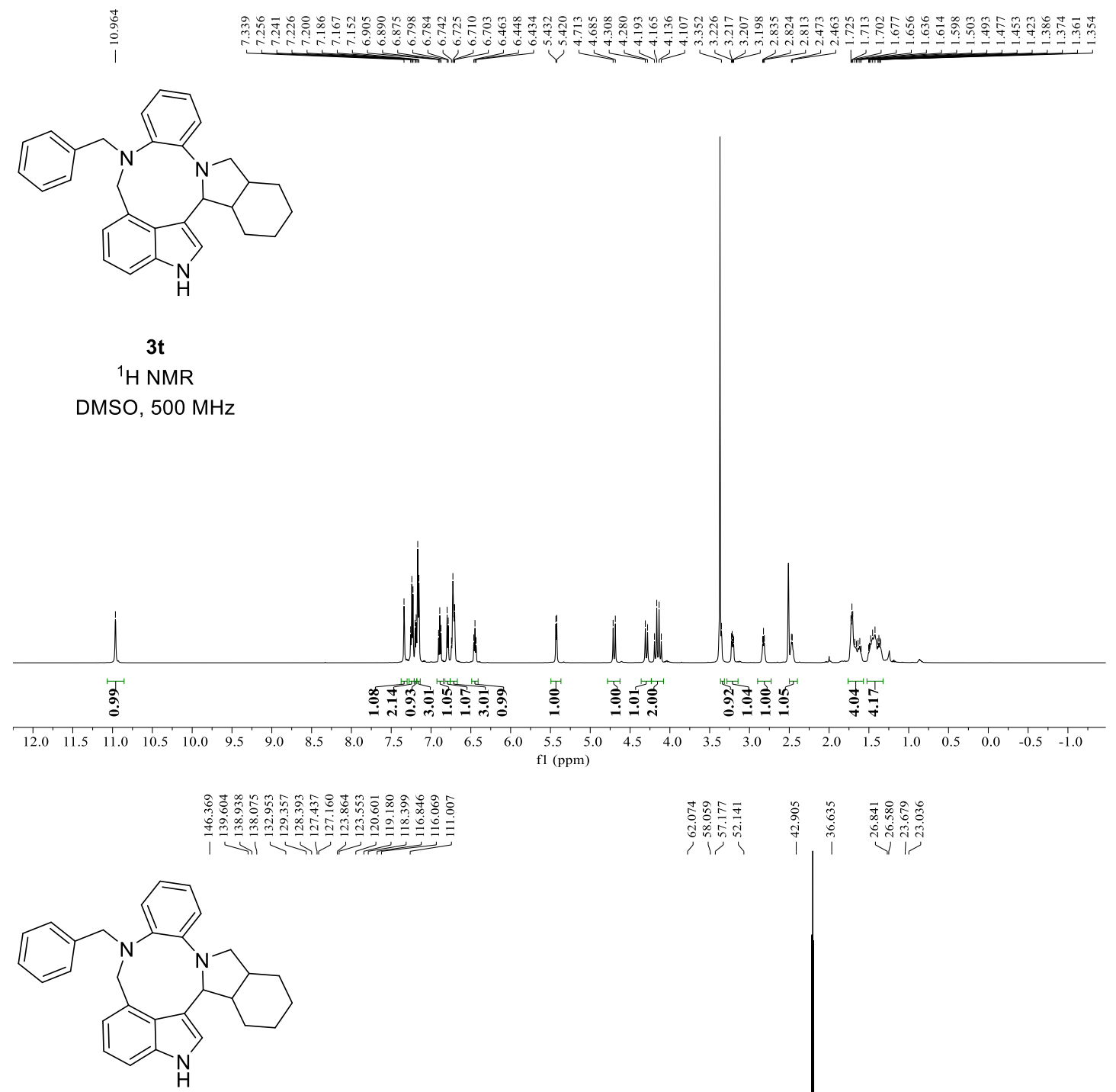

$3 t$

${ }^{13} \mathrm{C}$ NMR

DMSO, $125 \mathrm{MHz}$

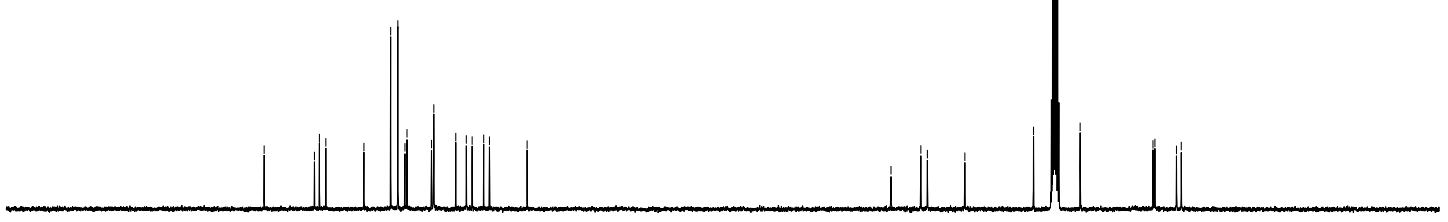

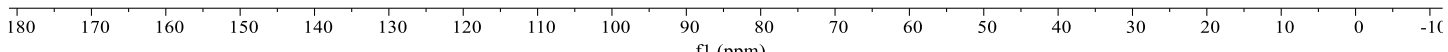




\section{X-ray Crystallography for 3a}

Compound 3a was dissolved in the solution of $n$-pentane and dichloromethane to form the saturated solution. After slow evaporation at room temperature, the single crystal was afforded.

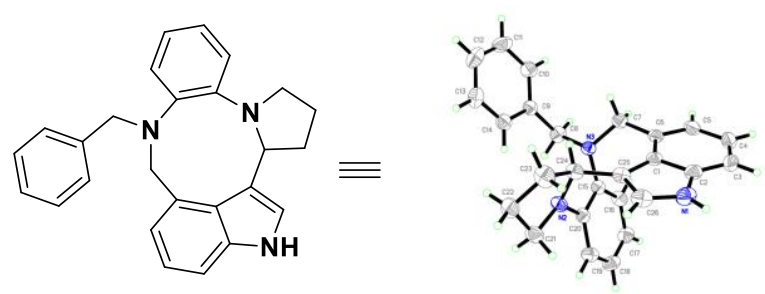

3a (CCDC 2077986)

exp_11786

Empirical formula

Formula weight

Temperature

Wavelength

Crystal system, space group

Unit cell dimensions

Volume

Z, Calculated density

Absorption coefficient

$\mathrm{F}(000)$

Crystal size

Theta range for data collection

Limiting indices

Reflections collected / unique

Completeness to theta $=67.207$

Refinement method

Data / restraints / parameters

Goodness-of-fit on $\mathrm{F}^{\wedge} 2$

Final $\mathrm{R}$ indices $[\mathrm{I}>2 \operatorname{sigma}(\mathrm{I})]$

$\mathrm{R}$ indices (all data)

Extinction coefficient

Largest diff. peak and hole
$\mathrm{C}_{26} \mathrm{H}_{25} \mathrm{~N}_{3}$

379.49

293(2) K

$1.54184 \mathrm{~A}$

Monoclinic, $\mathrm{P} 2(1) / \mathrm{c}$

$\mathrm{a}=8.8829(4) \mathrm{A} \quad$ alpha $=90 \mathrm{deg}$.

$\mathrm{b}=9.8022(5) \mathrm{A} \quad$ beta $=91.021(4) \mathrm{deg}$.

$\mathrm{c}=22.9519(10)$ A gamma $=90 \mathrm{deg}$. 1998.15(15) $\mathrm{A}^{\wedge} 3$

4, $1.261 \mathrm{Mg} / \mathrm{m}^{\wedge} 3$

$0.575 \mathrm{~mm}^{\wedge}-1$

808

$0.130 \times 0.120 \times 0.110 \mathrm{~mm}$

3.852 to $67.207 \mathrm{deg}$.

$-10<=\mathrm{h}<=6,-11<=\mathrm{k}<=11,-27<=\mathrm{l}<=27$

$6884 / 3583$ [R(int) $=0.0336]$

$99.9 \%$

Full-matrix least-squares on $\mathrm{F}^{\wedge} 2$

$3583 / 0 / 262$

1.002

$\mathrm{R} 1=0.0512, \mathrm{wR}_{2}=0.1139$

$\mathrm{R} 1=0.0823, \mathrm{wR} 2=0.1346$

$\mathrm{n} / \mathrm{a}$

0.137 and -0.224 e. $\mathrm{A}^{\wedge}-3$ 


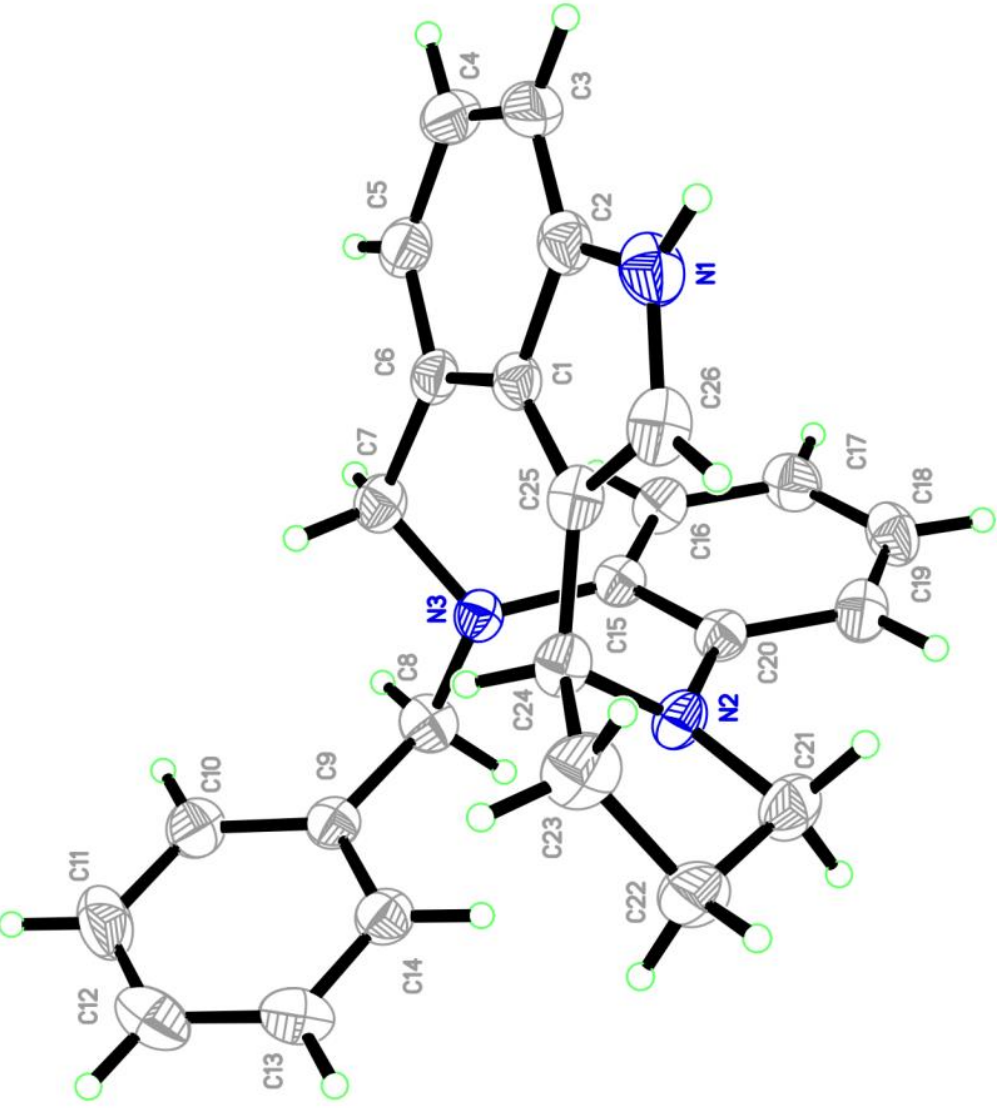

